\title{
Abundance analysis of the supergiant stars HD 80057 and HD 80404 based on their UVES Spectra
}

\author{
T. Tanrıverdi ${ }^{\mathrm{a}, *}$, Ö. Baştürk ${ }^{\mathrm{b}}$ \\ ${ }^{a}$ Niğde University, Faculty of Arts and Sciences, Department of Physics, TR-51240, Niğde, Turkey \\ ${ }^{b}$ Ankara University, Faculty of Science, Department of Astronomy and Space Sciences, TR-06100, \\ Tandoğan, Ankara, Turkey
}

\begin{abstract}
This study presents elemental abundances of the early A-type supergiant HD 80057 and the late A-type supergiant HD 80404. High resolution and high signal-to-noise ratio spectra published by the UVES Paranal Observatory Project (Bagnulo et al., 2003)1 were analysed to compute their elemental abundances using ATLAS9 (Kurucz, 1993, 2005; Sbordone et al., 2004). In our analysis we assumed local thermodynamic equilibrium. The atmospheric parameters of HD 80057 used in this study are from Firnstein \& Przybilla (2012), and that of HD 80404 are derived from spectral energy distribution, ionization equilibria of $\mathrm{Cr} \mathrm{I} / \mathrm{II}$ and $\mathrm{Fe} \mathrm{I} / \mathrm{II}$, and the fits to the wings of Balmer lines and Paschen lines as $T_{\text {eff }}=7700 \pm 150 \mathrm{~K}$ and $\log g=1.60 \pm 0.15$ (in cgs). The microturbulent velocities of HD 80057 and HD 80404 have been determined as $4.3 \pm 0.1$ and $2.2 \pm 0.7$ $\mathrm{km} \mathrm{s}^{-1}$. The rotational velocities are $15 \pm 1$ and $7 \pm 2 \mathrm{~km} \mathrm{~s}^{-1}$ and their macroturbulence velocities are $24 \pm 2$ and $2 \pm 1 \mathrm{~km} \mathrm{~s}^{-1}$. We have given the abundances of 27 ions of 20 elements for HD 80057 and 39 ions of 25 elements for HD 80404. The abundances are close to solar values, except for some elements ( $\mathrm{Na}, \mathrm{Sc}, \mathrm{Ti}, \mathrm{V}, \mathrm{Ba}$, and $\mathrm{Sr})$. We have found the metallicities $[\mathrm{M} / \mathrm{H}]$ for $\mathrm{HD} 80057$ and $\mathrm{HD} 80404$ as $-0.15 \pm 0.24$ and -0.02 \pm 0.20 dex, respectively. The evolutionary status of these stars are discussed and their

\footnotetext{
*e-mail: ttanriverdi@nigde.edu.tr

${ }^{1}$ Based on data obtained with the UVES Paranal Observatory Project (ESO DDT Program ID 266.D-
} $5655)$
\end{abstract}

Preprint submitted to New Astronomy

June 4, 2018 
nitrogen-to-carbon $(N / C)$ and nitrogen-to-oxygen $(N / O)$ ratios show that they are in their blue supergiant phase before the red supergiant region.

Keywords:

Stars: abundances - Stars: individual: HD 80057 - Stars: individual: HD 80404 -

Techniques: spectroscopic

\section{Introduction}

A-type supergiants are attractive astrophysical targets for chemical abundance studies. First of all, they are among the brightest stars at visual wavelengths, which makes them observable with low exposure times, high resolution and high signal-to-noise ratios $(\mathrm{S} / \mathrm{N})$. Moreover, their spectra are unblended, hence the abundances of numerous elements with consecutive ionization levels such as light elements, $\alpha$ process elements, iron group and s-process elements can be derived from studies of their atmospheres (Venn, 1995; Albavrak, 2000; Przybilla, 2002; Schiller \& Przybilla, 2008; Firnstein \& Przybilla, 2012; Tanrıverdi, 2013). With these results in hand, it is possible to understand their nature and the environments in which they exist, and thereby study galactic and extra-galactic abundance gradients and dispersions.

This paper is a continuation of analyses of early and late A-type supergiants started by Tanriverdi et al. (2004); Tanriverdi (2013). In this study, elemental abundance analyses of two A-type supergiants, HD 80057 and HD 80404 (iota Car), and their revised atmospheric parameters, are presented in detail. These analyses are based on spectra distributed by the UVES Paranal Observatory Project (Bagnulo et al., 2003).

\subsection{HD 80057}

HD 80057 (HR 3688, HIP 45481, SAO 221010) was classified as A1 Iab by (Firnstein \& Przybilla, 2012) (see Table 1 for more information). It is a member of the Vela OB1 association of stars, which is one of the largest OB star associations of the Galaxy, and is composed of numerous members (Reed, 2000). Although HD 80057 has been used as a photometric 
standard star, (Menzies et al., 1989; Cousins, 1990), and as a spectroscopic standard star for its radial velocities (Reed \& Kuhna, 1997; Gontcharov, 2006), a detailed study of its atmosphere was published only very recently by Firnstein \& Przybilla (2012). The authors determined the atmospheric parameters ( $T_{\text {eff }}$ and $\log g$ ) using spectroscopic indicators and spectroscopic data (see Table 2) in their study, as well as CNO abundances of HD 80057 computed using non-LTE methods.

\section{2. $H D 80404$}

iota Car (HD 80404, HR 3699, HIP 45556, SAO 236808) is an MK Standard, which is classified as A8 Ib (Malaroda, 1973; Monier \& Parthasarathy, 1999) (see Table 1 for more information). It is one of the brightest stars in the southern sky in the visual region of the electromagnetic spectrum. Adelman et al. (2000) listed it amongst the least variable Hipparcos targets, while Gray \& Garrison (1989) gave its Strömgren photometric parameters.

The atmosphere of iota Car was first studied in detail by Boiarchuk \& Liubimkov (1984), who gave its spectroscopic parameters as $7300 \pm 200 \mathrm{~K}$ for its effective temperature and $1.40 \pm 0.2$ for its surface gravity. Luck \& Lambert (1985) later gave elemental abundances for the star as well as revising its stellar parameters, finding $T_{\text {eff }}=$ $7500 \pm 200 \mathrm{~K}, \log g=0.90 \pm 0.3$, and micro \& macro-turbulent velocities of 2.5, and $1.0 \pm 0.5 \mathrm{~km} \mathrm{~s}^{-1}$, respectively. Next, Luck \& Lambert (1992) adopted the effective temperature found by Luck \& Lambert (1985) as $7500 \mathrm{~K}$, and found a value of $1.6 \pm 0.2$ for its surface gravity using the MARCS code of Gustafsson et al. (1975) from its Fe I/II ionization balance. Then, Takeda \& Takada-Hidai (1995) re-calculated the CNO abundances of iota Car using Luck \& Lambert (1985)'s atmospheric parameters and equivalent widths (EW).

Smiljanic et al. (2006), on the other hand, computed the effective temperature to be $7500 \pm 200 \mathrm{~K}$, surface gravity $2.40 \pm 0.25$, and micro-turbulent velocity $2.34 \pm 0.35$ $\mathrm{km} \mathrm{s}^{-1}$ based on their high-resolution spectroscopic observations using fits to the $\mathrm{H} \alpha$ 
wings, the $\mathrm{Fe}$ I/II ionization equilibrium and their photometric calibration (see Table22). They also determined its $\mathrm{C}, \mathrm{N}, \mathrm{O}$, and Fe abundances from their FEROS (Fiber-fed Extended Range Optical Spectrograph) spectra in the wavelength range 3500-9200 ̊ and a resolution, $\mathrm{R}=48000$. Later on, spectrophotometric observations (visual and near-infrared) of the object were presented by Ruban et al. (2006).

\section{The Spectra}

The UVES spectra used in this study were obtained from the UVES-POP database. They have high resolution $(\mathrm{R} \sim 80000)$ and $\mathrm{S} / \mathrm{N}$ ratios (for most of the spectra $\mathrm{S} / \mathrm{N}$ ratio 300-500 in V band). They cover a wavelength range of $\lambda \lambda$ 3040-10400 $\AA$ (Bagnulo et al., 2003)2. The ultraviolet (UV), visual and infrared (IR) parts of UVES spectra were used to determine the abundances of elements such as $\mathrm{C}, \mathrm{N}, \mathrm{O}, \mathrm{Mg}$ and $\mathrm{Al}$ (see Table 3). All spectra were continuum normalized using IRAF 3 task continuum. Then, the EWs of the identified lines were measured using the splot package within IRAF. Main sources for line identification are mentioned in Tanriverdi (2013). International Ultraviolet Explorer (IUE)'s flux-calibrated spectra were downloaded from MAST 14 archive. For spectrophotometry of HD 80404, low-dispersion and large aperture spectra, SWP36720 and $L W P 15980$, were used.

\section{Stellar Parameters}

The atmospheric models were produced using ATLAS9 (Kurucz, 1993; Sbordone et al.,

2004). LTE abundance analyses were performed based on EW measurements using the WIDTH9 code(Kurucz, 1993). The effective temperatures and surface gravities ( $T_{\text {eff }}$,

\footnotetext{
${ }^{2}$ http://www.eso.org/sci/observing/tools/uvespop.html

${ }^{3}$ IRAF is distributed by the National Optical Astronomy Observatory, which is operated by the Association of Universities for Research in Astronomy (AURA) under cooperative agreement with the National Science Foundation.

${ }^{4}$ http://archive.stsci.edu
} 
$\log g$ ) were determined via SED (Spectral Energy Distribution, see Fig.11) analyses, fits to Balmer and Paschen lines wings (see Figs.22\& 3) and from the ionization equilibria of $\mathrm{Cr} \mathrm{I} / \mathrm{II}$ and $\mathrm{Fe} \mathrm{I} / \mathrm{II}$ (see Fig. (4). The procedure used to determine these fundamental parameters are illustrated on the $T_{\text {eff }}-\log g$ plane in Fig. 4. The microturbulent velocity was determined by finding the value where the correlation between the derived abundances and the EWs $\left(\xi_{1}\right)$ was minimised, and the minimum scatter about the abundance mean $\left(\xi_{2}\right)$ was obtained (Blackwell et al., 1982). Microturbulent velocities were determined for HD 80057 and HD 80404 to be 4.30 and $2.20 \mathrm{~km} \mathrm{~s}^{-1}$, respectively. The derived microturbulent velocities of different species are given in Table 4. The rotational velocity and the macroturbulent velocity of HD 80057 are determined using synthetic spectra produced by SYNSPEC and SYNPLOT (Hubeny, 1988; Hubeny \& Lanz, 2011). The rotational and macroturbulence velocities for HD 80057 are $15 \pm 1 \mathrm{~km} \mathrm{~s}^{-1}$ and $24 \pm 2 \mathrm{~km} \mathrm{~s}^{-1}$ and those for HD 80404 are $7 \pm 2 \mathrm{~km} \mathrm{~s}^{-1}$ and $2 \pm 1 \mathrm{~km} \mathrm{~s}^{-1}$.

The SED in Fig. 1 was reproduced using ATLAS9 flux models. The spectrophotometric data were obtained from Ruban et al. (2006). The photometric data, angular diameter and $\mathrm{E}(\mathrm{B}-\mathrm{V})$ are given in Table 1 . The zero-points reported by Heber et al. (2002) were used to transform the various magnitudes into monochromatic fluxes. It was assumed that $y=V$ to transform $b-y, c_{1}$ and $m_{1}$ indexes to $u, v, b$ and $y$ magnitudes. The computed fluxes of HD $80404\left(T_{\text {eff }}=7700 \mathrm{~K}\right.$ and $\left.\log g=1.60\right)$ were also consistent with its SED. The synthetic spectrum was reproduced for $\mathrm{H}_{\delta}$ increasing and decreasing $\pm 150 \mathrm{~K}$ or $\pm 0.15 \mathrm{dex}, T_{\text {eff }}$, and $\log g$, respectively using SYNTHE(Kurucz \& Avrett, 1981). We also used the bluest part of the Balmer series. Different to the synthetic Balmer series spectrum, we also tried to determine the $T_{\text {eff }}, \log g$ pair of the Paschen series, for which we obtained a good fit. Paschen lines were previously used by Schiller \& Przybilla (2008) to determine the atmospheric parameters of Deneb. The excitation potentials of $\mathrm{Fe}_{\mathrm{I}}$ and $\mathrm{Fe}$ II lines in our abundance analysis ranged from $0.00 \mathrm{eV}$ to $10 \mathrm{eV}$. Their abundances and excitation potentials showed no correlation at $T_{\text {eff }}=$ 
$7700 \mathrm{~K}$ (see Fig. 5). The slope of the excitation potential to Fe abundances was -

$4.943 \times 10^{-3} \pm 3.764 \times 10^{-3} \mathrm{dex}^{-1}$. This is another method to determine the atmospheric parameters, such as $T_{\text {eff }}$. Moreover, the ionization equilibrium is also good tool to determine the stellar parameters. Ionization equilibrium for the consecutive ionization stages of $\mathrm{Cr} \mathrm{I} / \mathrm{II}$ and $\mathrm{Fe} \mathrm{I} / \mathrm{II}$ were fullfilled in the atmospheres of HD 80404. The errors in the determined values of $T_{\text {eff }}$ and $\log g$ were assumed to be $\pm 150 \mathrm{~K}$ and $\pm 0.15 \mathrm{dex}$, as determined from their $\mathrm{H} \delta$ fits, and the error of the microturbulent velocity value was assumed to be $0.7 \mathrm{~km} \mathrm{~s}^{-1}$, as obtained from microturbulence velocity determinations of individual elements in Table 4.

\section{The results of the abundance analysis}

The elemental abundances found in this study for HD 80057 and HD 80404 are presented in Table 3 and Fig. 6, together with a comparison of our results with solar composition and previous studies. The systematic error calculated for the abundances of HD 80404 are given in Table 5. The detailed abundances are given in Table 6, which also includes the elements used in the analysis, the wavelengths of the identified lines, gf values, and their references. The ionization equilibrium of different elements/ions for target stars are seen in Table 3

While the errors in $T_{\text {eff }}$ have the strongest effect on the abundances of $\mathrm{Mg}$ II, $\mathrm{Al}$ I, Fe I and Ba II, the error in $\log g$ affects most strongly those of $\mathrm{Mg}_{\text {II, }} \mathrm{S}_{\text {II, }} \mathrm{Ca}$ I and $\mathrm{Ca}$ II. This might be due to the fact that these species have the strongest lines with the large EWs.

The sum of CNO abundances of both stars have a solar value. For HD 80057, $\alpha$ process elements, except for $\mathrm{Si}$ and $\mathrm{Ca}$, are also under-abundant. $\mathrm{Al}, \mathrm{Sc}, \mathrm{Ti}$, and $\mathrm{V}$ are found to be under-abundant. However, $\mathrm{Cr}, \mathrm{Mn}, \mathrm{Fe}$, and $\mathrm{Ni}$ abundances are found to be closer to solar values. Sc, Ti, and V are susceptible to non-LTE effects, which can be ascribed to the value of their second ionization potentials. These are the lowest second 
ionisation potential in the Iron Group (Przybilla, 2002). The heavy elements (Sr, Y, Zr, Ba) are also underabundant with respect to solar abundances.

In the atmospheres of HD 80404; $\alpha$-process elements ( $\mathrm{Si}, \mathrm{S}$ and $\mathrm{Ca}$ ) and the light element $\mathrm{Al}$ are closer to solar values, $\mathrm{Mg}$ is deficient, however $\mathrm{Na}$ is overabundant. $\mathrm{Sc}, \mathrm{Ti}, \mathrm{V}, \mathrm{Cr}, \mathrm{Mn}, \mathrm{Fe}, \mathrm{Co}$ and $\mathrm{Ni}$ are all closer to solar values in the atmosphere of HD 80404. The heavy elements tend to have values slightly smaller than solar, Ba is overabundant(see Fig. 11).

\section{Results and Discussion}

As a result, the $[\mathrm{M} / \mathrm{H}]$ ratio of $\mathrm{HD} 80057$ is found to be $-0.15 \pm, 0.24$ dex when we exclude the over-abundant Na due to NLTE effects (Takeda \& Takada-Hidai, 1994; Venn, 1995; Takeda, 2008), the under-abundant Sc and Ti, which are all susceptible to non-LTE effects. The $[\mathrm{M} / \mathrm{H}]$ ratio of HD 80404 is estimated to be $-0.02 \pm 0.20$ dex when we exclude the over-abundant $\mathrm{Na}$ element. The $N / C, N / O$ and $\Sigma C N O$ values are given in Table 7

\subsection{Evolutionary Status}

Light-element, the sum of $C N O$ composition reflects mixing process present in the interior of the star. Both stars show a deficiency of $\mathrm{C}$ and $\mathrm{O}$, and an enrichment of $\mathrm{N}$. Hence, the combined $C N O$ abundances were found to be close to the solar value (see Table 7). Both stars also have solar metallicity. The values of $N / C$ and $N / O$ predicted from a linear interpolation of the closest isochrones are consistent with their calculated values (see Table 7). The $N / O$ ratio of HD 80057 is found to be slightly smaller than the theoretical value 0.52 .

The early CNO contamination in the surface layers of stars can be explained by rotating models. Rotation is an essential factor of stellar models that have a profound effect on the evolution of especially massive stars. Red giants or supergiants, whose progenitor is fast rotating progenitors, rotate six times faster and show N/C ratios three 
time higher than those formed by slow rotators (Przybilla et al., 2010; Georgy et al., 2013; Maeder et al., 2014). Georgy et al. (2013) provide extended data of stellar models including the mass range from 1.7 to $15 \mathrm{M}_{\odot}$ with three different metallicities and with nine different initial rotation velocity models. In the framework of their study, one can see that $\mathrm{C}, \mathrm{N}$, and $\mathrm{O}$ abundances varies with different initial rotating models. So, we take into account, initial rotational velocities, $N / C$ and $N / O$ ratios, besides $T_{\text {eff }}$ and $\log g$ in stellar evolution models.

In order to investigate the evolutionary states of our targets, we used the Geneva Stellar Model (Georgy et al., 2013) interactive tools 5 to interpolate between the existing evolutionary tracks that would lead to models with parameters matching those of our targets. We used the relevant parameters $\left(\log \mathrm{T}_{e f f}\right.$ and $\left.\log g\right)$ from Firnstein \& Przybilla (2012) for HD 80057 and our own measurements for HD 80404.

We experimented by interpolating models for different masses of grids that covered a wide range of masses between 1.7 and $15 \mathrm{M}_{\odot}$. We kept the metallicity at solar composition $(\mathrm{Z}=0.014)$, and the rotation rate at $v / v_{\text {crit }}=0.0$ to 0.95 in each case.

We searched for the probable evolutionary track, which would be the one with the closest agreement with the atmospheric parameters of our stars (e.g. effective temperatures and surface gravities). We found that both of our stars have masses between $10 \mathrm{M}_{\odot}$ and $14 \mathrm{M}_{\odot}$. While HD 80057 has a mass consistent with the track for $13 \mathrm{M}_{\odot}$, HD 80404 is very close to the track for $12 \mathrm{M}_{\odot}$. The latter result is based on its fundamental parameters (Fig. 7), which assumes solar composition and rotation with values of $\Omega / \Omega_{c r i t}=0.60,0.50$ (see Table 7). These gave globally good fit to stars from the main-sequence to the positions of the blue supergiants before the red supergiant phase in the Hertzsprung-Russell Diagram (hereafter HRD) (Georgy et al., 2013).

On the $\log T_{\text {eff }}-\log g$ plane, we also computed isochrones using the same online tools and selected the ones to which our stars' parameters had the closest matches

\footnotetext{
${ }^{5}$ http://obswww.unige.ch/Recherche/evoldb/index/
} 
(Fig. 8). Our results indicate ages close to 16 Myrs for HD 80057 and 19 Myrs for HD 80404. When the errors in the measurements of the parameters, expressed as the error bars in Fig. 8, were considered, a good estimate was obtained for ages between 12.5 and 25 Myrs for both of our stars. The abundance ratios $N / C$ that we computed in this study (2.45 for HD 80057 and 1.57 for HD 80404) are somewhat consistent (within the uncertainty limits) with the stars' positions on the $\log T_{\text {eff }}-\log g$ plane, for which the expected $N / C$ ratios are printed next to each of the isochrones in Fig 8 , The derived values of the $N / C$ and $N / O$ ratios from the isochrones are given in Table 7 . These ratios reveal that $C N O$ mixing processes are active, and that these stars are at the Blue Supergiant (BSG) phase in their evolution prior to the Red Supergiant (RSG) phase, as according to Saio et al. (2013).

\section{Acknowledgements}

This research utilised the SIMBAD database, which is operated at CDS, Strasbourg, France. This work, made use of the MAST-IUE archive (http://archive.stsci.edu/iue/) of SAO/NASA ADS, is based on data obtained from UVES Paranal Observatory Project (ESO DDT Program ID 266.D-5655), of the VALD database, operated at Uppsala University, the Institute of Astronomy RAS in Moscow, and the University of Vienna. Atomic data compiled in the DREAM data base (E. Biemont, P. Palmeri \& P. Quinet, Astrophys. Space Sci. 269-270, 635, 1999) were extracted via VALD (Kupka et al., 1999, A\&AS 138, 119, and references therein). The authors thank the anonymous referee, whose useful comments helped to improve this work.

\section{References}

Adelman, S.J., Cay, I.H., Cay, M.T., \& Kocer, D. 2000. IBVS 4947, 1.

Albayrak, B. 2000, A\&A, 364, 237 
Aldenius, M., Tanner, J. D., Johansson, S., Lundberg, H., \& Ryan, S. G. 2007. A\&A $461,767$.

Arellano Ferro, A., Mantegazza, L., 1996. A\&A 315, 542.

Bagnulo, S., Jehin, E., Ledoux, C., et al. 2003. The Messenger 114, 10.

Biémont, E., Grevesse, N., Hannaford, P., Lowe, R.M., 1981. ApJ 248, 867.

Biémont, E., Grevesse, N., Faires, L.M., Marsden, G., Lawler, J.E., Whaling, W., 1989. A\&A 209, 391.

Blackwell, D. E., Shallis, M. J., \& Simmons, G. J. 1982. MNRAS 199, 33.

Blackwell-Whitehead, R., \& Bergemann, M. 2007. A\&A 472, L43.

Boiarchuk, A.A., Liubimkov, L.S., 1984. Astrofizika 20, 85.

Brage, T., Wahlgren, G. M., Johansson, S. G., Leckrone, D. S., \& Proffitt, C. R. 1998, ApJ, 496, 1051

Corliss, C. H., \& Bozman, W. R. 1962. NBS Monograph, Washington: US Department of Commerce, National Bureau of Standards, $\_$c1962,

Cousins, A. W. J. 1987. South African Astronomical Observatory Circulars 11, 93.

Cousins, A. W. J. 1990. South African Astronomical Observatory Circulars 14, 55.

Davidson, M. D., Snoek, L. C., Volten, H., \& Doenszelmann, A. 1992. A\&A 255, 457.

Davis, J., Jacob, A. P., Robertson, J. G., et al. 2009, MNRAS, 394, 1620

Ducati, J.R., 2002. CDS/ADC Collection of Electronic Catalogues, 2237.

Przybilla, N., Firnstein, M., Nieva, M. F., Meynet, G., \& Maeder, A. 2010, A\&A, 517, A38

Firnstein, M., Przybilla, N., 2012. A\&A 543, A80. 
Fuhr, J. R., Martin, G. A., \& Wiese, W. L. 1988. J. Phys. Chem. Ref. Data 17.

Fuhr, J.R., Wiese, W.L., 2002. in: Lide D.R. (ed.), CRC Handbook of Chemistry and Physics, 86th edition(CRC Press, Boca Raton).

Fuhr, J.R. and Wiese, W.L., 2006. J. Phys. Chem. Ref. Data 35, 1669.

Georgy, C., Ekström, S., Granada, A., et al. 2013, A\&A, 553, A24

Gontcharov, G. A. 2006. Astronomy Letters 32, 759.

Gray, R. O., Garrison, R. F. 1989. ApJS 70, 623.

Grevesse, N. 1984. Physica Scripta Volume T, 8, 49.

Grevesse, N., Sauval, A. J. 1996. Standard Abundances, in: Holt S.S. \& Sonneborn, G. (Eds.), Cosmic Abundances: Proceedings of the 6th Annual October Astrophysics Conference, ASP Conf.Ser. 99, pp.117-126.

Grevesse, N., \& Sauval, A. J. 1998. Space Sci. Rev. 85, 161.

Gustafsson, B., Bell, R. A., Eriksson, K., \& Nordlund, A. 1975. A\&A 42, 407.

Hannaford, P., Lowe, R.M., Grevesse, N., Biemont, E., Whaling, W. 1982. ApJ 261, 736.

Hauck, B., \& Mermilliod, M. 1998. A\&AS 129, 431.

Heber, U., Moehler, S., Napiwotzki, R., Thejll, P., \& Green, E. M. 2002. A\&A 383, 938.

Hubeny, I. 1988, Computer Physics Communications, 52, 103

Hubeny, I., \& Lanz, T. 2011, Astrophysics Source Code Library, 1109.022

Jönsson, G., Kröll, S., Persson, A., \& Svanberg, S. 1984. Phys. Rev. A 30, 2429.

Kling, R., \& Griesmann, U. 2000. ApJ 531, 1173. 
Kling, R., Schnabel, R., \& Griesmann, U. 2001. ApJS 134, 173.

Kupka, F., Piskunov, N., Ryabchikova, T. A., Stempels, H. C., \& Weiss, W. W. 1999. A\&AS 138, 119.

Kupka, F. G., Ryabchikova, T. A., Piskunov, N. E., Stempels, H. C., \& Weiss, W. W. 2000. Baltic Astronomy 9, 590.

Kurucz, R. L. 1979. ApJS 40, 1.

Kurucz, R. L., \& Avrett, E. H. 1981, SAO Special Report, 391

Kurucz, R. 1993. ATLAS9 Stellar Atmosphere Programs and 2 km/s grid. Kurucz CDROM No. 13. Cambridge, Mass.: Smithsonian Astrophysical Observatory, 1993., 13.

Kurucz, R. L. 1995. Astrophysical Applications of Powerful New Databases, 78, 205.

Kurucz, R. L. 2005. Memorie della Societa Astronomica Italiana Supplementi 8, 14.

Lanz, T., Artru, M.-C., 1985. Phys. Scr 32, 115.

Lawler, J.E., Dakin, J.T., 1989. Journal of the Optical Society of America B Optical Physics B 6, 1457.

Lawler, J. E., , G., \& Sneden, C. 2001. ApJ 556, 452.

Lawler, J.E., Wickliffe, M.E., den Hartog, E.A., Sneden, C., 2001. ApJ 563, 1075.

Ljung, G., Nilsson, H., Asplund, M., \& Johansson, S. 2006. A\&A 456, 1181.

Luck, R.E., Lambert, D.L., 1985. ApJ 298, 782.

Luck, R. E., \& Lambert, D. L. 1992. ApJS 79, 303.

Maeder, A., Przybilla, N., Nieva, M.-F., et al. 2014, A\&A, 565, A39

Malaroda, S. 1973. PASP 85, 328. 
Martin, G. A., Fuhr, J. R., \& Wiese, W. L. 1988. New York: American Institute of Physics (AIP) and American Chemical Society, 1988,

Meggers, W. F., Corliss, C. H., \& Scribner, B. F. 1975, Tables of spectral-line intensities. Part I, II_- arranged by elements., by Meggers, W. F.; Corliss, C. H.; Scribner, B. F.. NBS Monogr. 145, Part I, II, 15+387 P.; 15+213 p.,

Menzies, J. W., Cousins, A. W. J,Banfield, R. M., Laing, J. D. 1989. South African Astronomical Observatory Circular 13, 1.

Monier, R., \& Parthasarathy, M. 1999. A\&A 341, 117.

Nilsson, H., Ljung, G., Lundberg, H., Nielsen, K.E., 2006. A\&A 445, 1165.

Palmeri, P., Quinet, P., Wyart, J.-F., \& Biémont, E. 2000. Phys. Scr 61, 323.

Pickering, J.C., Thorne, A.P. and Perez, R. 2001. ApJS 132, 403.

Pickering, J.C., Thorne, A.P. and Perez, R. 2002. ApJS 138, 247.

Piskunov, N. E., Kupka, F., Ryabchikova, T. A., Weiss, W. W., \& Jeffery, C. S. 1995. A\&AS 112, 525 .

Przybilla, N. 2002, Ph.D.Thesis, Ludwig-Maximilians-Universität München, Germany

Raassen, A.J.J., Pickering, J.C., Uylings, P.H.M., 1998. A\&A 130, 541.

Reed, B. C., Kuhna, K. M. 1997. AJ 113, 823.

Reed, B. C. 2000. AJ 119, 1855.

Ruban, E. V., Alekseeva, G. A., Arkharov, A. A., et al. 2006. Astronomy Letters 32, 604.

Ryabchikova, T. A., Piskunov, N. E., Kupka, F., \& Weiss, W. W. 1997. Baltic Astronomy 6, 244. 
Saio, H., Georgy, C., \& Meynet, G. 2013. MNRAS 433, 1246.

Sbordone, L., Bonifacio, P., Castelli, F., \& Kurucz, R. L. 2004. Memorie della Societa Astronomica Italiana Supplementi 5, 93.

Schiller, F., \& Przybilla, N. 2008. A\&A 479, 849.

Schulz-Gulde, E. 1969. J. Quant. Spec. Radiat. Transf. 9, 13.

Smiljanic, R., Barbuy, B., de Medeiros, J. R., \& Maeder, A. 2006. A\&A 449, 655.

Snow, T. P., Lamers, H. J. G. L. M., Lindholm, D. M., \& Odell, A. P. 1994. ApJS 95, 163.

Takeda, Y. 2008. MNRAS 388, 913.

Takeda, Y., \& Takada-Hidai, M. 1994, PASJ, 46, 395

Takeda, Y., \& Takada-Hidai, M. 1995. PASJ 47, 169.

Tanriverdi, T., 2013. 2013. New A 25, 50T.

Tanriverdi, T., Adelman, S. J., \& Albayrak, B. 2004, The A-Star Puzzle, 224, 869

Tetzlaff, N., Neuhäuser, R., \& Hohle, M. M. 2011. MNRAS 410, 190.

van der Wal, S. E., \& van Genderen, A. M. 1988. A\&A, 195, 172.

van Leeuwen, F. 2007. A\&A 474, 653.

Venn, K. A. 1995, ApJS, 99, 659

Wiese, W.L., Smith, M.W., and Miles, B.M., 1969. Atomic Transition Probabilities. Vol. 2: Sodium through Calcium. A Critical Data Compilation, NSRDS-NBS 22. Government Printing Office, Washington DC

Wiese, W.L., Fuhr, J.R., Deters, T.M., 1996. J. Phys. Chem. Ref. Data Mon. 7. 
Wiese, W.L., Martin, G.A., 1980. Wavelengths and Transition Probabilities for Atoms and Atomic Ions. Part 2: Transition Probabilities, NSRDS-NBS 68, Government Printing Office, Washington, DC

Wiese, W. L., \& Fuhr, J. R. 2007. J. Phys. Chem. Ref. Data 36, 1287. 


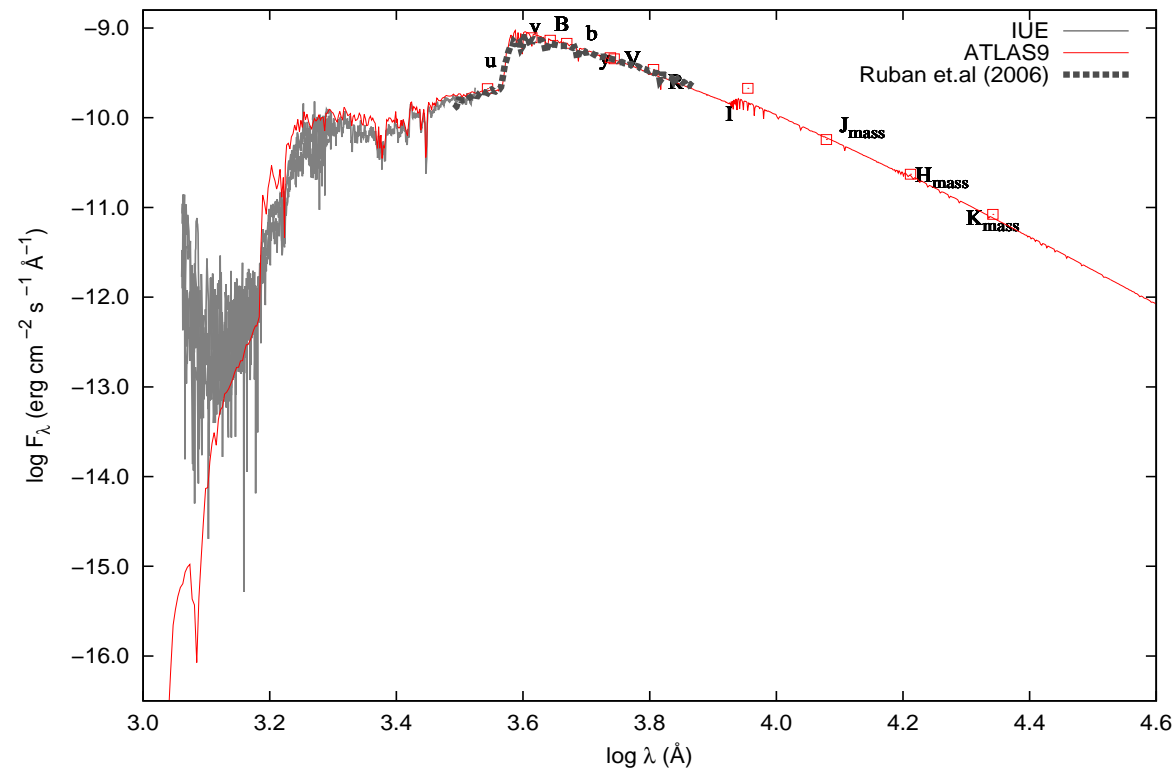

Figure 1: A comparison of the observed and computed fluxes $\left(T_{\text {eff }}=7700 \mathrm{~K}, \log g=1.60\right)$ for HD 80404 . ATLAS9 model flux, ATLAS9 reddened model flux, IUE spectra, the spectrophotometric data of Ruban et al. (2006) and the photometric data are also given. 

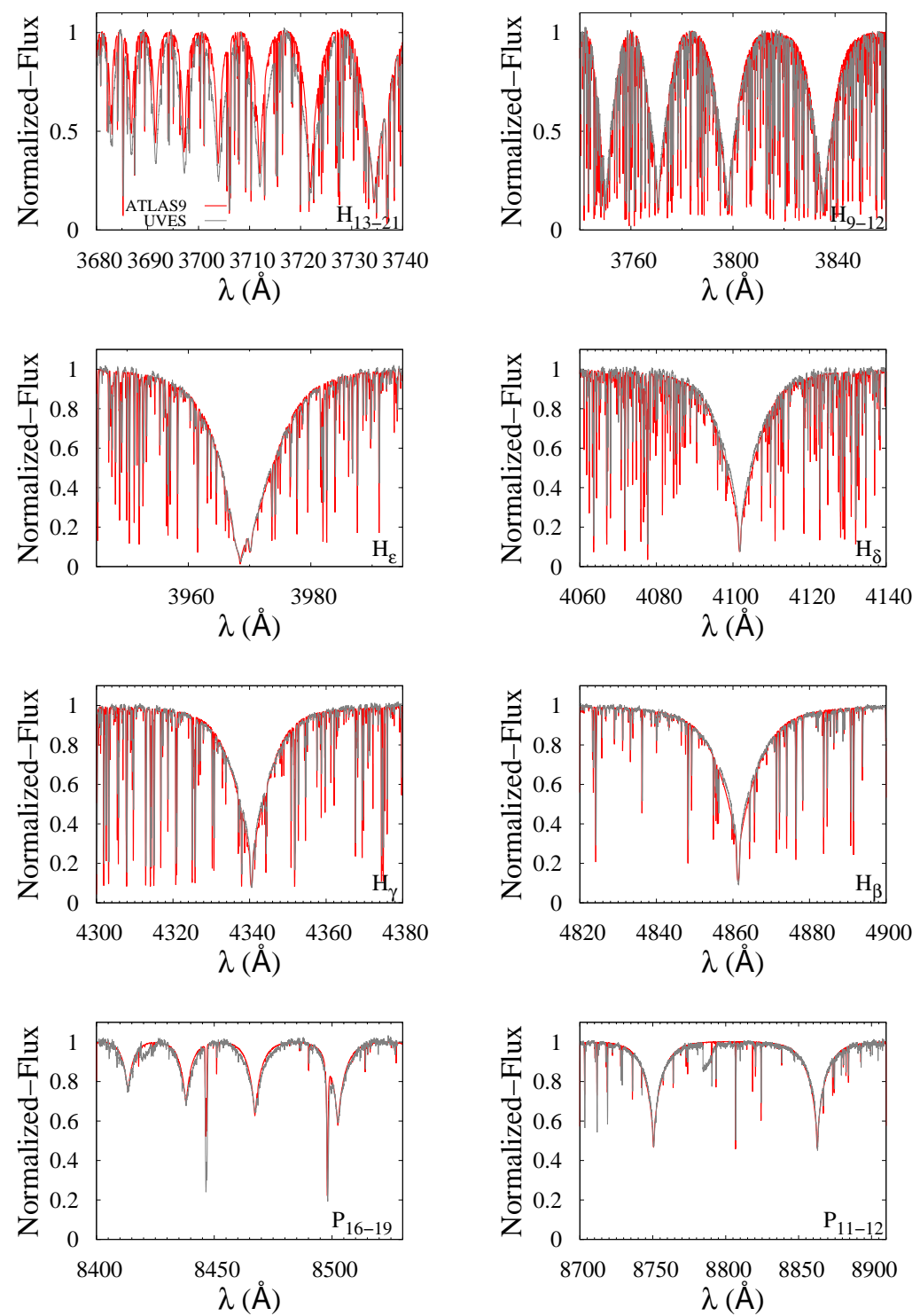

Figure 2: Synthetic spectrum fits of Balmer and Paschen series in the UVES spectra of HD 80404 for $T_{\text {eff }}=$ $7700 \mathrm{~K}, \log g=1.60$ pair. 

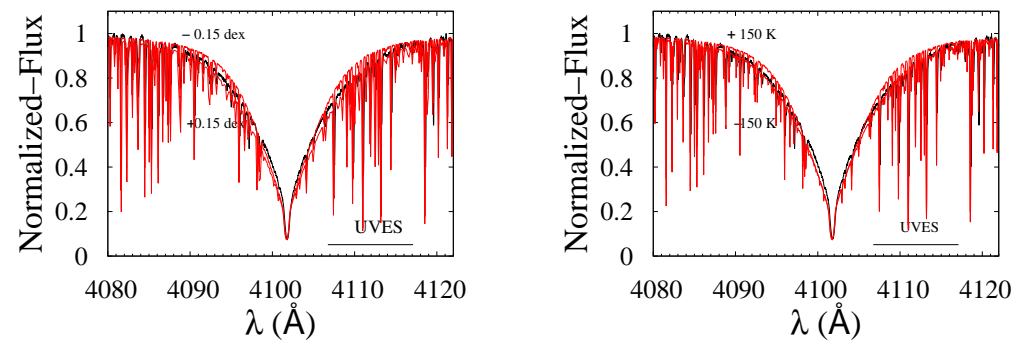

Figure 3: Synthetic spectrum fits of $\mathrm{H}_{\delta}$ lines in HD 80404 spectra using $T_{\text {eff }}=7700( \pm 150 \mathrm{~K}), \log g=1.60$ $( \pm 0.15$ dex $)$, synthetic spectrum and UVES spectrum.

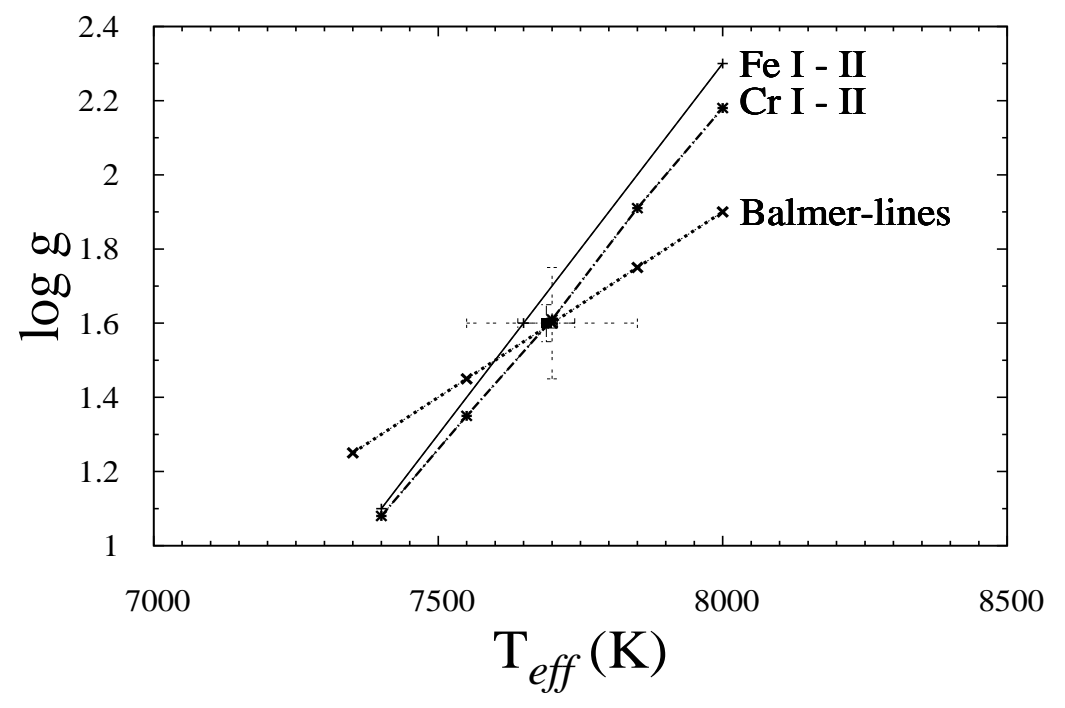

Figure 4: $T_{\text {eff }}-\log g$ planes for $T_{\text {eff }}=7700 \mathrm{~K}, \log g=1.60$ of HD 80404 based on Cr I/II, Fe I/II ionization levels and Balmer line fits. 

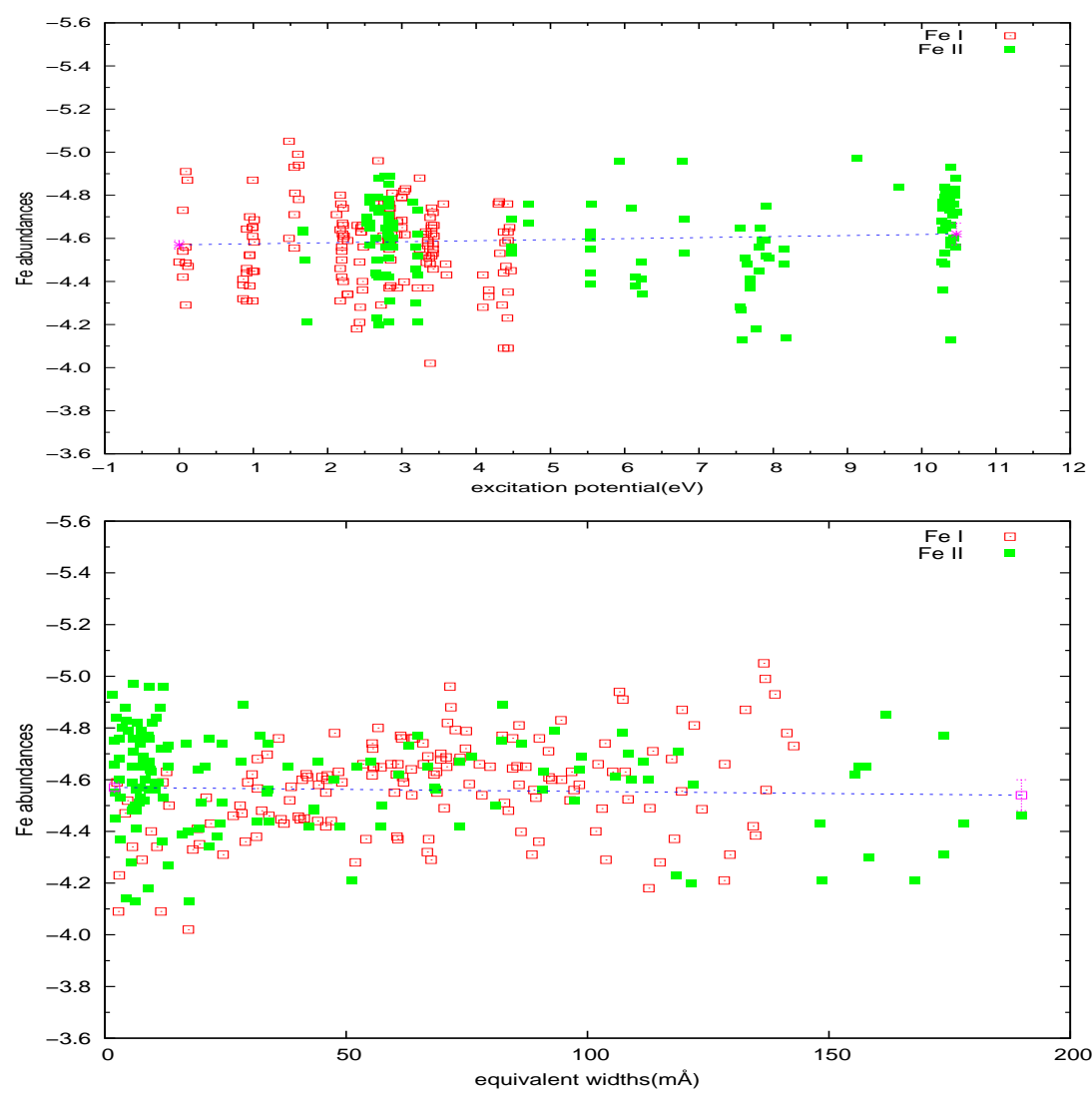

Figure 5: Fe abundances versus their equivalent widths and the different excitation potential values are plotted. The correlation between excitation potentials and Fe abundances is minimum at $T_{\text {eff }}=7700 \mathrm{~K}$. 


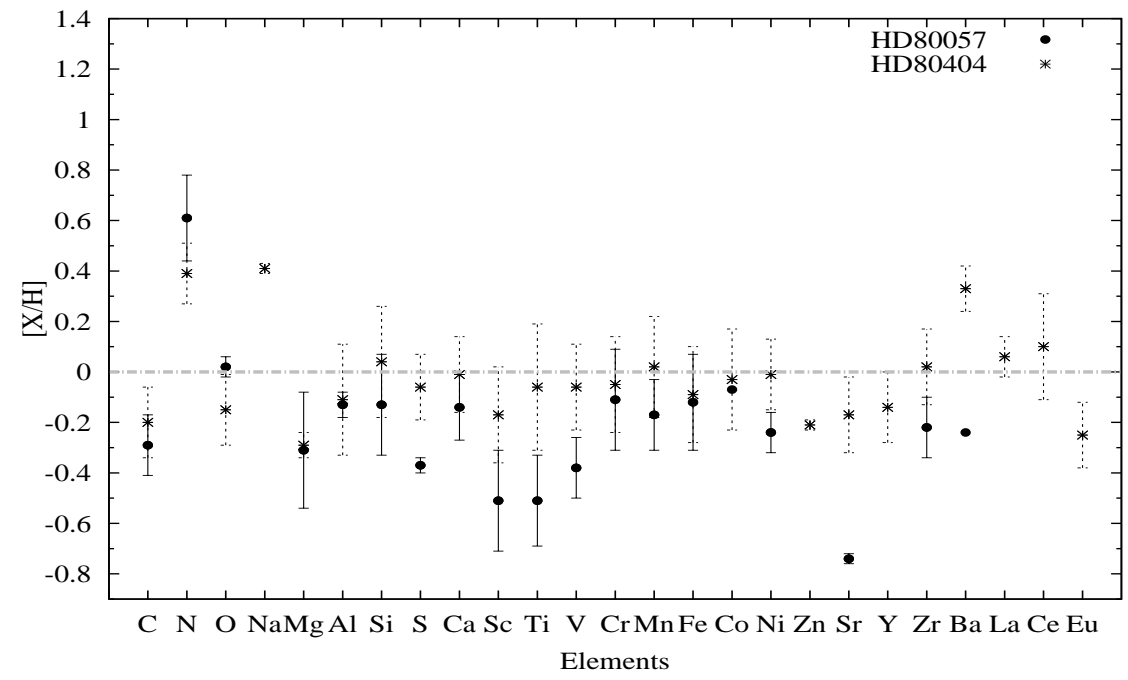

Figure 6: The chemical abundances of HD 80057 and HD 80404 compared to the solar values in Grevesse \& Sauval (1996).

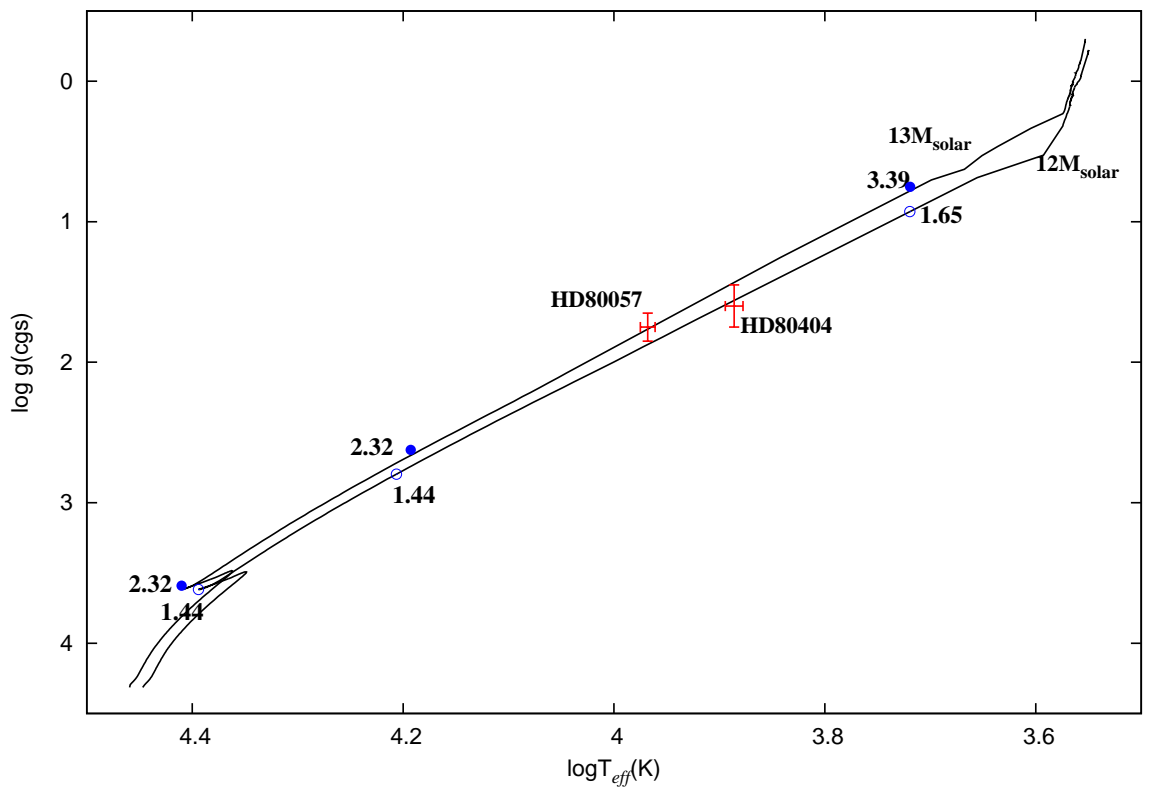

Figure 7: The positions of HD 80057 and HD 80404 on $\log T_{\text {eff }} \log g$ plane, and evolutionary tracks for 12 and $13 \mathrm{M}_{\odot}$, found by interpolation between existing tracks of Geneva stellar models Georgy et al. (2013). We labelled certain $N / C$ values (by mass ratio) on each of the tracks for comparison with our measurements. 




Figure 8: The positions of HD 80057 and HD 80404 on $\log T_{\text {eff }}-\log g$ plane, and two isochrones $\left(10^{7.2}\right.$ and $10^{7.3}$ years) computed by making use of Geneva stellar models Georgy et al. (2013) in solid curves. We labelled certain $N / C$ values (by mass ratio) on each of the isochrones for comparison with our measurements. 
Table 1: Stellar parameters of HD 80057 and HD 80404 from other authors.

\begin{tabular}{|c|c|c|}
\hline & HD 80057 & HD 80404 \\
\hline \multicolumn{3}{|l|}{ Basic } \\
\hline Name & $\ldots$ & $\iota \mathrm{Car}$ \\
\hline Association & Vela OB1 ${ }^{a}$ & $\ldots$ \\
\hline Spectral type & $\mathrm{A} 1-\mathrm{Iab}^{b}$ & $\mathrm{~A} 8-\mathrm{Ib}^{f}$ \\
\hline Distance ( kpc) & $1.449 \pm 0.819,1.839^{c, b}$ & $0.235 \pm 0.005^{c}$ \\
\hline Radial velocity $\left(\mathrm{km} \mathrm{s}^{-1}\right)$ & $25.7 \pm 2.0^{d}$ & $12.0 \pm 0.3^{d}$ \\
\hline \multicolumn{3}{|l|}{ Atmospheric } \\
\hline$T_{\text {eff }}(\mathrm{K})$ & $9300 \pm 150^{b}$ & $7500 \pm 200^{g}$ \\
\hline $\log g(\mathrm{cgs})$ & $1.75 \pm 0.10^{b}$ & $2.40 \pm 0.25^{g}$ \\
\hline$\xi\left(\mathrm{km} \mathrm{s}^{-1}\right)$ & $5 \pm 1^{b}$ & $2.35 \pm 0.35^{g}$ \\
\hline$\zeta\left(\mathrm{km} \mathrm{s}^{-1}\right)$ & $27 \pm 5^{b}$ & \\
\hline$v \sin i\left(\mathrm{~km} \mathrm{~s}^{-1}\right)$ & $13 \pm 5^{b}$ & $10.0 \pm 0.6^{d}$ \\
\hline \multicolumn{3}{|l|}{ Photometric } \\
\hline \multicolumn{3}{|l|}{ (Johnson) } \\
\hline$V$ & $6 .^{m} 044 \pm 0^{m} .016^{b}$ & $2 .^{m} 26^{h}$ \\
\hline$B-V$ & $0 .^{m} 285 \pm 0^{m} .006^{b}$ & $0 \cdot^{m} 18^{h}$ \\
\hline$U-B$ & $-0 .^{m} 117 \pm 0^{m} .021^{b}$ & $0 .^{m} 16^{h}$ \\
\hline \multicolumn{3}{|l|}{ (Strömgren) } \\
\hline$b-y$ & $0.267 \pm 0.003^{e}$ & $0.123 \pm 0.007^{i}$ \\
\hline$m_{1}$ & $-0.009 \pm 0.011^{e}$ & $0.130 \pm 0.009^{i}$ \\
\hline$c_{1}$ & $0.861 \pm 0.007^{e}$ & $1.542 \pm 0.011^{i}$ \\
\hline$M_{V}$ & $-6 .^{m} 40 \pm 0 \cdot^{m} 18^{a}$ & $-5 .^{m} 1^{j}$ \\
\hline$(m-M)_{0}$ & 9. ${ }^{m} 52 \pm 0 \cdot^{m} 18^{a}$ & $7 .^{m} 2^{j}$ \\
\hline$M_{\mathrm{bol}}$ & $-6 .^{m} 49 \pm 0 \cdot^{m} 18 \quad a$ & $-5 \cdot^{m} 3^{k}$ \\
\hline$B C$ & $-0 . .^{m} 09$ & $0 .^{m} 21^{k}$ \\
\hline$E(B-V)$ & $0 .^{m} 32 \pm 0.02^{b}$ & $0 .^{m} 13^{j}$ \\
\hline$\theta_{D}(m a s)$ & $\ldots$ & $1 .^{m} 55 \pm 0.12^{l}$ \\
\hline
\end{tabular}

Reed (2000), $\quad$ Firnstein \& Przybilla (2012), $\quad$ van Leeuwen (2007), ${ }^{a}$ Gontcharov $(2006)$, ${ }^{t}$ Hauck \& Mermilliod (1998), ${ }^{f}$ Tetzlaff et al. (2011), ${ }^{8}$ Smilianic et al. (2006), ${ }^{h}$ Ducati (2002), Arellano Ferro \& Mantegazza (1996), Snow et al. (1994) van der Wal \& van Genderen (1988) Davis et al. (2009) 


\begin{tabular}{|c|c|c|c|c|c|c|}
\hline Source & $\begin{array}{l}T_{\text {eff }}(\mathrm{K}) \\
\end{array}$ & $\overline{l o g} g$ &  & $\overline{\zeta \zeta\left(\mathrm{km} \mathrm{s}^{-1}\right)}$ & Spectra & Method \\
\hline & $\underline{\mathrm{HD}} 80057$ & & & & & \\
\hline \multirow[t]{3}{*}{ Firnstein \& Przybilla (2012) } & $9300 \pm 150$ & $1.75 \pm 0.10$ & $5 \pm 1$ & 27 & FEROS, R 40000 & NLTE \\
\hline & & & & & $3500-9300 \AA ., \mathrm{S} / \mathrm{N}>150$ & \\
\hline & iota $\mathrm{Car}$ & & & & & \\
\hline Boiarchuk \& Liubimkov (1984) & $7300 \pm 200$ & $1.40 \pm 0.10$ & $\ldots$ & $\ldots$ & reciprocal dispersion, $2 \AA / \mathrm{mm}$ & LTE, $\underline{\text { Kurucz }}$ \\
\hline \multirow[t]{2}{*}{ Luck \& Lambert (1985) } & 7500 & 0.90 & 2.5 & 1 & & LTE, Fe I/ II \\
\hline & & & & & & ionization balance \\
\hline Luck \& Lambert (1992) & 7500 & 1.6 & 2.2 & 1 & CTIO, R 18000 & LTE \\
\hline \multirow[t]{2}{*}{ Smilianic et al. (2006) } & 7500 & 2.40 & 2.34 & $\ldots$ & FEROS, $\mathrm{R}=48000$ & LTE, $H_{\alpha}$, and Fe I/II \\
\hline & & & & & $3500-9200 \AA, \mathrm{S} / \mathrm{N}>150$ & ionization balance \\
\hline
\end{tabular}


Table 3: The comparison of derived abundances of target stars relative to the solar values and literature.

\begin{tabular}{|c|c|c|c|c|c|c|c|c|}
\hline \multirow[b]{2}{*}{ Species } & \multirow[t]{2}{*}{ Solar $^{1}$} & \multicolumn{3}{|c|}{ HD 80057} & \multicolumn{4}{|c|}{ HD 80404} \\
\hline & & $\mathrm{n}$ & This Study & $\mathrm{FP}^{2,3}$ & $\mathrm{n}$ & This Study & $\mathrm{n}$ & $\mathrm{LL}^{4,5}$ \\
\hline $\mathrm{C}_{\mathrm{I}}$ & -3.45 & 5 & $-3.72 \pm 0.22$ & $-3.78 \pm 0.08$ & 12 & $-3.65 \pm 0.14$ & . & -3.67 \\
\hline $\mathrm{C}_{\text {II }}$ & -3.45 & 2 & $-3.78 \pm 0.04$ & $-3.72 \pm 0.13$ & $\ldots$ & $\ldots$ & $\ldots$ & $\ldots$ \\
\hline $\mathrm{N}_{\mathrm{I}}$ & -4.03 & 1 & $-3.42 \pm 0.17$ & $-3.66 \pm 0.04$ & 3 & $-3.52 \pm 0.14$ & $\ldots$ & -3.72 \\
\hline $\mathrm{N}_{\text {II }}$ & -4.03 & $\ldots$ & $\ldots$ & -3.71 & $\ldots$ & $\ldots$ & $\ldots$ & $\ldots$ \\
\hline $\mathrm{O}_{\mathrm{I}}$ & -3.13 & 3 & $-3.11 \pm 0.04$ & $\ldots$ & 14 & $-3.34 \pm 0.24$ & 3 & -3.38 \\
\hline $\mathrm{Na}$ I & -5.67 & $\ldots$ & $\ldots$ & $\ldots$ & 3 & $-5.26 \pm 0.02$ & 2 & $-5.19 \pm 0.03$ \\
\hline $\mathrm{Mg}_{\mathrm{I}}$ & -4.42 & 2 & $-4.78 \pm 0.22$ & $-4.58 \pm 0.06$ & 1 & -4.67 & 10 & $-4.35 \pm 0.23$ \\
\hline $\mathrm{Mg}_{\text {II }}$ & -4.42 & 5 & $-4.71 \pm 0.08$ & $-4.60 \pm 0.06$ & 4 & $-4.72 \pm 0.05$ & $\ldots$ & $\ldots$ \\
\hline $\mathrm{Al}_{\mathrm{I}}$ & -5.53 & 2 & $-6.08 \pm 0.05$ & $\ldots$ & 2 & $-5.56 \pm 0.22$ & 1 & -5.58 \\
\hline $\mathrm{Al}$ II & -5.53 & 1 & -6.03 & $\ldots$ & 1 & -5.81 & $\ldots$ & $\ldots$ \\
\hline $\mathrm{Si}$ I & -4.45 & $\ldots$ & $\ldots$ & $\ldots$ & 2 & $-4.16 \pm 0.00$ & 27 & $-4.23 \pm 0.16$ \\
\hline Si II & -4.45 & 11 & $-4.57 \pm 0.20$ & $\ldots$ & 7 & $-4.66 \pm 0.22$ & $\ldots$ & $\ldots$ \\
\hline$S_{I}$ & -4.67 & $\ldots$ & $\ldots$ & $\ldots$ & 3 & $-4.63 \pm 0.08$ & 9 & $-4.63 \pm 0.14$ \\
\hline $\mathrm{S}_{\text {II }}$ & -4.67 & 2 & $-5.04 \pm 0.03$ & $\ldots$ & 3 & $-4.82 \pm 0.10$ & $\ldots$ & $\ldots$ \\
\hline $\mathrm{Ca}$ & -5.64 & 1 & -5.75 & $\ldots$ & 24 & $-5.64 \pm 0.15$ & 12 & $-5.57 \pm 0.17$ \\
\hline Ca II & -5.64 & 2 & $-5.79 \pm 0.16$ & $\ldots$ & 3 & $-5.75 \pm 0.02$ & 3 & $-5.54 \pm 0.40$ \\
\hline Sc II & -8.83 & 7 & $-9.34 \pm 0.20$ & $\ldots$ & 26 & $-9.00 \pm 0.20$ & 5 & $-9.05 \pm 0.13$ \\
\hline Ti I & -6.98 & $\ldots$ & $\ldots$ & $\ldots$ & 40 & $-6.89 \pm 0.24$ & $\ldots$ & $\ldots$ \\
\hline Ti II & -6.98 & 43 & $-7.49 \pm 0.18$ & $\ldots$ & 59 & $-7.15 \pm 0.21$ & 1 & -7.05 \\
\hline$V_{\text {I }}$ & -8.00 & $\ldots$ & $\ldots$ & $\ldots$ & 4 & $-8.05 \pm 0.11$ & $\ldots$ & $\ldots$ \\
\hline $\mathrm{V}_{\text {II }}$ & -8.00 & 7 & $-8.38 \pm 0.12$ & $\ldots$ & 25 & $-8.06 \pm 0.18$ & $\ldots$ & $\ldots$ \\
\hline $\mathrm{Cr}_{\mathrm{I}}$ & -6.33 & $\ldots$ & $\ldots$ & $\ldots$ & 40 & $-6.40 \pm 0.19$ & $\ldots$ & $\ldots$ \\
\hline Cr II & -6.33 & 50 & $-6.44 \pm 0.17$ & $\ldots$ & 59 & $-6.37 \pm 0.18$ & $\ldots$ & $\ldots$ \\
\hline Mn I & -6.61 & $\ldots$ & $\ldots$ & $\ldots$ & 25 & $-6.56 \pm 0.22$ & $\ldots$ & $\ldots$ \\
\hline $\mathrm{Mn}_{\text {II }}$ & -6.61 & 8 & $-6.78 \pm 0.14$ & $\ldots$ & 11 & $-6.66 \pm 0.11$ & $\ldots$ & $\ldots$ \\
\hline $\mathrm{Fe}_{\mathrm{I}}$ & -4.50 & 25 & $-4.70 \pm 0.21$ & $\ldots$ & 161 & $-4.59 \pm 0.18$ & 61 & $-4.29 \pm 0.19$ \\
\hline $\mathrm{Fe}_{\text {II }}$ & -4.50 & 105 & $-4.62 \pm 0.18$ & $\ldots$ & 139 & $-4.58 \pm 0.20$ & 12 & $-4.30 \pm 0.16$ \\
\hline $\mathrm{Fe}_{\text {III }}$ & -4.50 & 3 & $-4.69 \pm 0.06$ & $\ldots$ & $\ldots$ & $\ldots$ & $\ldots$ & $\ldots$ \\
\hline CoI & -7.08 & $\ldots$ & $\ldots$ & $\ldots$ & 7 & $-7.17 \pm 0.15$ & $\ldots$ & $\ldots$ \\
\hline Co II & -7.08 & 1 & -7.15 & $\ldots$ & 5 & $-6.95 \pm 0.20$ & $\ldots$ & $\ldots$ \\
\hline $\mathrm{Ni}$ & -5.75 & $\ldots$ & $\ldots$ & $\ldots$ & 17 & $-5.73 \pm 0.12$ & $\ldots$ & $\ldots$ \\
\hline Ni II & -5.75 & 3 & $-5.99 \pm 0.08$ & $\ldots$ & 3 & $-5.92 \pm 0.08$ & $\ldots$ & $\ldots$ \\
\hline $\mathrm{Zn}$ I & -7.40 & $\ldots$ & $\ldots$ & $\ldots$ & 2 & $-7.61 \pm 0.02$ & $\ldots$ & $\ldots$ \\
\hline Sr II & -9.03 & 2 & $-9.77 \pm 0.02$ & $\ldots$ & 4 & $-9.20 \pm 0.15$ & $\ldots$ & $\ldots$ \\
\hline $\mathrm{Y}_{\mathrm{II}}$ & -9.76 & $\ldots$ & $\ldots$ & $\ldots$ & 15 & $-9.90 \pm 0.10$ & 1 & $-9.61 \pm 0.03$ \\
\hline Zr II & -9.40 & 3 & $-9.62 \pm 0.12$ & $\ldots$ & 17 & $-9.42 \pm 0.15$ & $\ldots$ & $\ldots$ \\
\hline Ba II & -9.87 & 1 & -10.11 & $\ldots$ & 4 & $-9.54 \pm 0.09$ & 2 & $-9.40 \pm 0.09$ \\
\hline La II & -10.83 & $\ldots$ & $\ldots$ & $\ldots$ & 12 & $-10.77 \pm 0.08$ & $\ldots$ & $\ldots$ \\
\hline $\mathrm{Ce}_{\text {II }}$ & -10.42 & $\ldots$ & $\ldots$ & $\ldots$ & 9 & $-10.32 \pm 0.21$ & $\ldots$ & $\ldots$ \\
\hline Eu II & -11.49 & $\ldots$ & $\ldots$ & $\ldots$ & 4 & $-11.74 \pm 0.13$ & 2 & $-11.12 \pm 0.02$ \\
\hline$T_{\text {eff }}(\mathrm{K})$ & & . & 9300 & $\cdots$ & & 7700 & & \\
\hline $\log g(\mathrm{cgs})$ & & $\ldots$ & 1.75 & $\ldots$ & & 1.60 & & \\
\hline
\end{tabular}


Table 4: Microturbulence determinations from various elements/ions

\begin{tabular}{|c|c|c|c|c|c|c|}
\hline Elemen & $\mathrm{n}$ & $\begin{array}{l}\xi_{1}(\text { scatter }) \\
\mathrm{km} \mathrm{s}^{-1}\end{array}$ & $\log \left(\mathrm{N} / \mathrm{N}_{T}\right)$ & $\begin{array}{l}\xi_{2} \text { (slope) } \\
\mathrm{km} \mathrm{s}^{-1}\end{array}$ & $\log \left(\mathrm{N} / \mathrm{N}_{T}\right)$ & Reference \\
\hline \multicolumn{7}{|c|}{ HD 80057} \\
\hline $\begin{array}{r}\text { Fe II } \\
\text { a) } \\
\text { stc }\end{array}$ & 105 & $\begin{array}{l}4.3 \\
4.4 \\
0.1\end{array}$ & $-4.62 \pm 0.18$ & 4.4 & $-4.62 \pm 0.18$ & $\begin{array}{l}\mathrm{KX}+\mathrm{N} 4 \\
+\mathrm{VALD}\end{array}$ \\
\hline \multicolumn{7}{|c|}{ HD 80404} \\
\hline \multicolumn{2}{|c|}{ adopted } & $\begin{array}{l}4.1 \\
4.1\end{array}$ & $-3.71 \pm 0.15$ & 4.0 & $-3.71 \pm 0.15$ & WF \\
\hline $\begin{array}{l}\mathrm{Ca} \mathrm{I} \\
\quad \text { ado }\end{array}$ & $d^{39}$ & $\begin{array}{l}2.2 \\
2.2\end{array}$ & $-5.67 \pm 0.12$ & 2.2 & $-5.67 \pm 0.12$ & $\mathrm{FW}+\mathrm{WS}$ \\
\hline \multicolumn{2}{|c|}{ adopted } & $\begin{array}{l}2.0 \\
2.0\end{array}$ & $-4.56 \pm 0.16$ & 2.0 & $-4.56 \pm 0.16$ & $\begin{array}{l}\mathrm{KX}+\mathrm{N} 4 \\
+\mathrm{VALD}\end{array}$ \\
\hline $\begin{array}{l}\text { Fe II } \\
\text { ado }\end{array}$ & $d^{139}$ & $\begin{array}{l}2.3 \\
2.4\end{array}$ & $-4.63 \pm 0.17$ & 2.4 & $-4.59 \pm 0.18$ & $\begin{array}{l}\mathrm{KX}+\mathrm{N} 4 \\
+\mathrm{VALD}\end{array}$ \\
\hline \multicolumn{2}{|c|}{ adopted } & $\begin{array}{l}1.7 \\
1.7\end{array}$ & $-6.36 \pm 0.18$ & 1.7 & $-6.36 \pm 0.18$ & $\mathrm{MF}$ \\
\hline $\begin{array}{l}\mathrm{Cr} \text { II } \\
\text { ado }\end{array}$ & $d^{58}$ & $\begin{array}{l}2.0 \\
2.0\end{array}$ & $-6.32 \pm 0.17$ & 2.0 & $-6.32 \pm 0.17$ & $\begin{array}{l}\mathrm{MF}+\mathrm{KX} \\
+\mathrm{NL}\end{array}$ \\
\hline \multicolumn{2}{|c|}{ adopted } & $\begin{array}{l}1.8 \\
1.9\end{array}$ & $-9.02 \pm 0.10$ & 1.9 & $-9.02 \pm 0.10$ & $\mathrm{~B}+\mathrm{WM}$ \\
\hline \multicolumn{2}{|c|}{ adopted } & $\begin{array}{l}1.7 \\
1.7\end{array}$ & $-9.77 \pm 0.13$ & 1.8 & $-9.77 \pm 0.14$ & HL \\
\hline \multicolumn{2}{|c|}{ adopted } & $\begin{array}{l}1.7 \\
1.7\end{array}$ & $-9.33 \pm 0.12$ & 1.7 & $-9.33 \pm 0.12$ & $\mathrm{LN}+\mathrm{BG}$ \\
\hline & & $\begin{array}{l}2.2 \\
0.7\end{array}$ & & & & \\
\hline $\begin{array}{l}\text { Referen } \\
\mathrm{HL}=\mathrm{H} \\
\mathrm{FW}=\mathrm{F} \\
\mathrm{KX}=\mathrm{K} \\
\mathrm{NL}=\mathrm{N} \\
\mathrm{MF}=\mathrm{F} \\
\mathrm{WF}=\mathrm{V} \\
\mathrm{VALD}, \\
\text { Kupka }\end{array}$ & $\begin{array}{l}\text { of gf } \\
\text { naforc } \\
\text { r \& W } \\
\text { ucz (1 } \\
\text { son et } \\
\text { ret al } \\
\text { se et } \\
\text { LD2 }\end{array}$ & $\begin{array}{l}\text { values:B=B } \\
\text { et al. }(1982) \\
\text { ese }(2002) \text { a } \\
\text { 95), } \mathrm{LN}=\mathrm{L} \\
\text { 1. (2006), N } \\
1988) \text { and } \\
(1996) \text {; WI } \\
\text { ta= Piskuno } \\
\text { 9), Kupka et }\end{array}$ & $\begin{array}{l}\text { ge et al. (199 } \\
\text { Fuhr et al. } \\
\text { ng et al. (200 } \\
=\text { Fuhr \& Wi } \\
\text { rtin et al. (19) } \\
=\text { Wiese \& N } \\
\text { et al. (1995), } \\
\text {. (2000); }\end{array}$ & $\begin{array}{l}88) \\
\text { e } \\
\text { (2006); } \\
\text { rtin }(1980) \\
\text { vabchikov }\end{array}$ & $\begin{array}{l}\text { WS = Wiese } \\
\text { et al. (1997), }\end{array}$ & al. (1969); \\
\hline
\end{tabular}


Table 5: Error reasons for the abundances of HD 80404

\begin{tabular}{lccccc}
\hline \hline Species & $\sigma\left(T_{\text {eff }}\right)$ & $\Delta(\log g)$ & $\Delta(\xi)$ & EW & $\sigma_{\text {TOTAL }}$ \\
& $(+150 \mathrm{~K})$ & $(+0.15 \mathrm{dex})$ & $\left(+0.7 \mathrm{~km} \mathrm{~s}^{-1}\right)$ & $(10 \% \mathrm{EW})$ & \\
\hline C I & 0.06 & -0.04 & -0.02 & 0.07 & 0.10 \\
N I & 0.06 & 0.01 & 0.04 & 0.08 & 0.08 \\
O I & 0.00 & 0.01 & -0.02 & 0.04 & 0.04 \\
Na I & 0.11 & -0.07 & -0.03 & 0.06 & 0.14 \\
Mg I & 0.13 & -0.08 & -0.14 & 0.02 & 0.20 \\
Mg II & 0.00 & 0.02 & -0.09 & 0.03 & 0.10 \\
Al I & 0.15 & -0.07 & -0.31 & -0.13 & 0.37 \\
Al II & -0.05 & 0.07 & -0.02 & 0.03 & 0.09 \\
Si I & 0.12 & -0.07 & -0.01 & 0.04 & 0.15 \\
Si II & -0.02 & 0.04 & -0.10 & 0.01 & 0.11 \\
S I & 0.11 & -0.07 & -0.01 & 0.05 & 0.14 \\
S II & -0.10 & 0.18 & -0.02 & 0.06 & 0.19 \\
Ca I & 0.17 & -0.10 & -0.05 & 0.07 & 0.22 \\
Ca II & 0.06 & -0.10 & -0.07 & 0.08 & 0.12 \\
Sc II & 0.10 & -0.01 & -0.14 & 0.12 & 0.21 \\
Ti I & 0.16 & -0.08 & -0.01 & 0.00 & 0.19 \\
Ti II & 0.10 & 0.02 & -0.16 & 0.16 & 0.25 \\
V I & 0.16 & -0.08 & -0.01 & 0.04 & 0.18 \\
V II & 0.08 & 0.00 & -0.09 & 0.09 & 0.15 \\
Cr I & 0.16 & -0.07 & -0.04 & 0.04 & 0.19 \\
Cr II & 0.05 & 0.01 & -0.11 & 0.10 & 0.16 \\
Mn I & 0.14 & -0.08 & -0.05 & 0.07 & 0.18 \\
Mn II & 0.03 & 0.01 & -0.03 & 0.05 & 0.07 \\
Fe I & 0.15 & -0.09 & -0.13 & 0.13 & 0.25 \\
Fe II & 0.06 & 0.06 & -0.05 & 0.13 & 0.16 \\
Co I & 0.17 & -0.07 & -0.03 & 0.06 & 0.20 \\
Co II & 0.02 & -0.04 & -0.17 & 0.06 & 0.19 \\
Ni I & 0.13 & -0.08 & -0.02 & 0.05 & 0.16 \\
Ni II & 0.04 & 0.02 & -0.04 & 0.07 & 0.09 \\
Zn I & 0.14 & -0.07 & 0.00 & 0.05 & 0.16 \\
Sr II & 0.17 & -0.04 & -0.33 & 0.18 & 0.41 \\
Y II & 0.04 & -0.01 & -0.10 & 0.10 & 0.15 \\
Zr II & 0.03 & 0.00 & 0.06 & 0.08 & 0.10 \\
Ba II & -0.08 & 0.12 & -0.30 & 0.19 & 0.38 \\
La II & 0.14 & -0.04 & -0.03 & 0.06 & 0.16 \\
Ce II & 0.13 & -0.03 & -0.02 & 0.05 & 0.14 \\
Eu II & 0.17 & -0.04 & -0.02 & 0.05 & 0.18 \\
\hline$\sigma_{\text {tot. }}^{2}=\sigma_{\text {teff }}^{2}+\sigma_{\text {logg }}^{2}+\sigma_{\text {EW }}^{2}+\sigma_{\xi}^{2}$ & & & \\
& & & & &
\end{tabular}


Table 6: Elemental Abundances of HD 80057 and HD 80404

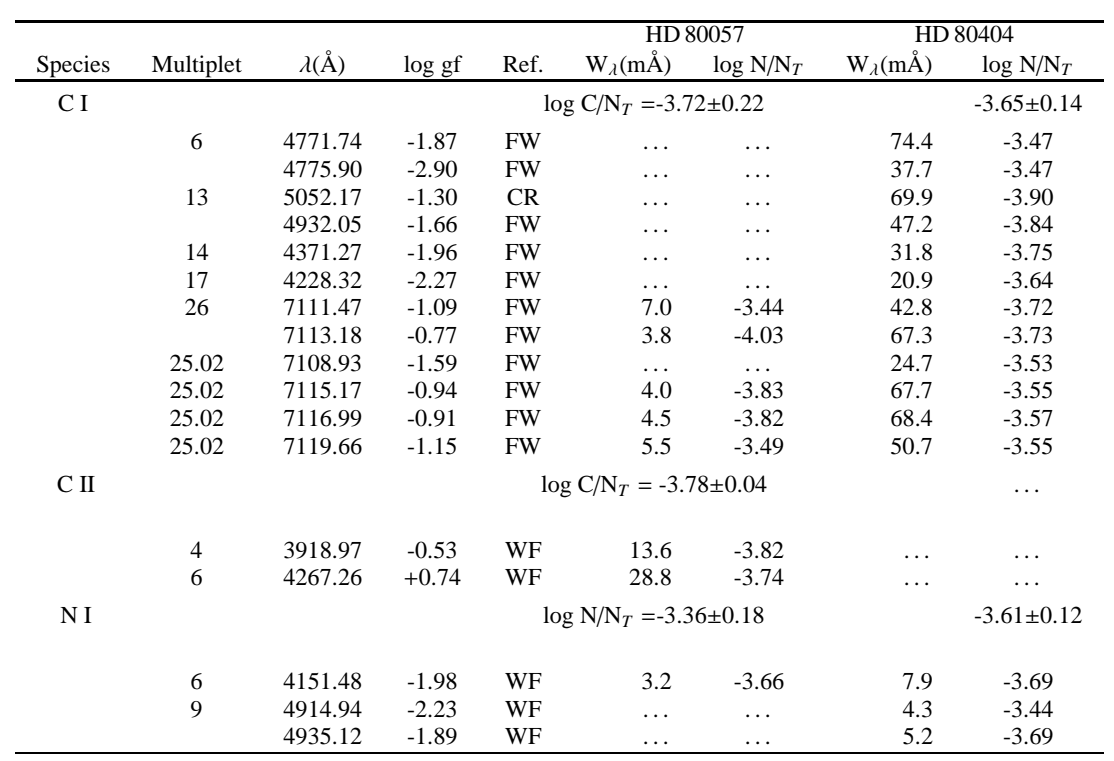

Note: gf value references follow:

$\mathrm{AT}=$ Aldenius et al. (2007); B= Brage et al. (1998) BB= Blackwell-Whitehead \& Bergemann (2007);

$\mathrm{BG}=$ Biemont et al. $(1981)$, Biemont et al. $(1989) ; \mathrm{CB}=$ Corliss \& Bozman (1962);CR = Wiese \& Fuhr $(2007)$

DS = Davidson et al. (1992) FW = Fuhr \& Wiese (2002) and Fuhr et al. (1988);HL = Hannaford et al. (1982)

$\mathrm{JK}=$ Jönsson et al. (1984) KG $=$ Kling \& Griesmann (2000); KS = Kling et al. (2001);KX = Kurucz (1995)

LA $=$ Lanz \& Artru (1985); $; \mathrm{LB}=$ Lawler et al. (2001) LD $=$ Lawler \& Dakin (1989); LN = Liung et al. (2006)

LW $=$ Lawler et al. (2001); MC $=$ Meggers et al. (1975) $; \mathrm{NL}=$ Nilsson et al. 2006

N4 $=$ Fuhr \& Wiese (2006); MF = Fuhr et al. (1988) and Martin et al. (1988);RP $=$ Raassen et al. (1998)

$\mathrm{PT}=$ Pickering et al. (2001) Pickering et al. (2002); $\mathrm{PQ}=$ Palmeri et al. $\mid 2000) ; \mathrm{SG}=$ Schulz-Gulde (1969)

$\mathrm{WF}=$ Wiese et al. (1996); WM = Wiese \& Martin (1980); WS = Wiese et al. (1969);

VALD, VALD2 data=Piskunov et al. (1995), Ryabchikova et al. (1997), Kupka et al. (1999), Kupka et al. (2000)

-The lines marked with * are ignored in average calculations.

-This table is given electronically. 
Table 7: The derived abundances and stellar parameters of HD 80057 and HD 80404 based on isochrones and evolutionary tracks of Georgy et al. (2013) for $\Omega / \Omega_{\text {crit }}=0.65,0.50$ and our abundance analysis.

\begin{tabular}{lcc}
\hline & HD80057 & HD80404 \\
\hline$\Omega$ & 0.60 & 0.50 \\
$\mathrm{~N} / \mathrm{C}$ & $2.44 \pm 0.91$ & $1.58 \pm 0.07$ \\
predicted & $2.39 \pm 0.05$ & $1.48 \pm 0.01$ \\
$\mathrm{~N} / \mathrm{O}$ & $0.43 \pm 0.17$ & $0.50 \pm 0.30$ \\
predicted & $0.52 \pm 0.01$ & $0.49 \pm 0.12$ \\
$\Sigma C N O$ & $0.07 \pm 0.10$ & $-0.05 \pm 0.08$ \\
$\mathrm{M} / \mathrm{H}$ & $-0.15 \pm 0.24$ & $-0.02 \pm 0.20$ \\
$v_{\text {crit }}$ & $147 \pm 1$ & $131 \pm 3$ \\
$v_{\text {sini }}$ & $25 \pm 3$ & $21 \pm 1$ \\
$R / R_{\odot}$ & $86 \pm 3$ & $96 \pm 10$ \\
$M^{\text {spec }} / M_{\odot}$ & $13 \pm 1$ & $13 \pm 2$ \\
$M^{\text {ZAMS }} / M_{\odot}$ & $13 \pm 1$ & $12 \pm 2$ \\
$M^{\text {evol }} / M_{\odot}$ & $13.00 \pm 0.30$ & $11.80 \pm 0.20$ \\
$\log L / L_{\odot}$ & $4.63 \pm 0.04$ & $4.46 \pm 0.02$ \\
\hline
\end{tabular}


Table A1: Elemental Abundances of HD 80057 and HD 80404

\begin{tabular}{|c|c|c|c|c|c|c|c|c|}
\hline \multirow[b]{2}{*}{ Species } & \multirow[b]{2}{*}{ Multiplet } & \multirow[b]{2}{*}{$\lambda(\AA)$} & \multirow[b]{2}{*}{$\log g f$} & \multirow[b]{2}{*}{ Ref. } & \multicolumn{2}{|c|}{ HD 80057} & \multicolumn{2}{|c|}{ HD 80404 } \\
\hline & & & & & $\mathrm{W}_{\lambda}(\mathrm{m} \AA)$ & $\log \mathrm{N} / \mathrm{N}_{T}$ & $\mathrm{~W}_{\lambda}(\mathrm{m} \AA)$ & $\log \mathrm{N} / \mathrm{N}_{T}$ \\
\hline \multirow[t]{13}{*}{$\mathrm{C}_{\mathrm{I}}$} & & & & & $\mathrm{C} / \mathrm{N}_{T}=-3.72$ & & & $-3.65 \pm 0.14$ \\
\hline & 6 & 4771.74 & -1.87 & $\mathrm{FW}$ & & $\ldots$ & 74.4 & -3.47 \\
\hline & & 4775.90 & -2.90 & $\mathrm{FW}$ & $\ldots$ & $\ldots$ & 37.7 & -3.47 \\
\hline & 13 & 5052.17 & -1.30 & $\mathrm{CR}$ & $\ldots$ & $\ldots$ & 69.9 & -3.90 \\
\hline & & 4932.05 & -1.66 & $\mathrm{FW}$ & & $\ldots$ & 47.2 & -3.84 \\
\hline & 14 & 4371.27 & -1.96 & FW & $\ldots$ & $\ldots$ & 31.8 & -3.75 \\
\hline & 17 & 4228.32 & -2.27 & $\mathrm{FW}$ & & & 20.9 & -3.64 \\
\hline & 26 & 7111.47 & -1.09 & $\mathrm{FW}$ & 7.0 & -3.44 & 42.8 & -3.72 \\
\hline & & 7113.18 & -0.77 & FW & 3.8 & -4.03 & 67.3 & -3.73 \\
\hline & 25.02 & 7108.93 & -1.59 & $\mathrm{FW}$ & $\ldots$ & & 24.7 & -3.53 \\
\hline & 25.02 & 7115.17 & -0.94 & FW & 4.0 & -3.83 & 67.7 & -3.55 \\
\hline & 25.02 & 7116.99 & -0.91 & $\mathrm{FW}$ & 4.5 & -3.82 & 68.4 & -3.57 \\
\hline & 25.02 & 7119.66 & -1.15 & FW & 5.5 & -3.49 & 50.7 & -3.55 \\
\hline $\mathrm{C}_{\mathrm{I}}$ & & & & & $\mathrm{C} / \mathrm{N}_{T}=-3.78$ & & & $\ldots$ \\
\hline & 4 & 3918.97 & -0.53 & WF & 13.6 & -3.82 & $\ldots$ & $\ldots$ \\
\hline & 6 & 4267.26 & +0.74 & $\mathrm{WF}$ & 28.8 & -3.74 & $\ldots$ & $\ldots$ \\
\hline NI & & & & & $\mathrm{N} / \mathrm{N}_{T}=-3.36$ & & & $-3.61 \pm 0.12$ \\
\hline & 6 & 4151.48 & -1.98 & WF & 3.2 & -3.66 & 7.9 & -3.69 \\
\hline & 9 & 4914.94 & -2.23 & WF & $\ldots$ & . & 4.3 & -3.44 \\
\hline & & 4935.12 & -1.89 & WF & $\ldots$ & $\ldots$ & 5.2 & -3.69 \\
\hline OI & & & & & $\mathrm{O} / \mathrm{N}_{T}=-3.1$ & & & $-3.34 \pm 0.24$ \\
\hline & 3 & 3947.29 & -2.10 & WF & $\ldots$ & $\ldots$ & 23.7 & -3.53 \\
\hline & & 3947.48 & -2.29 & $\mathrm{WF}$ & $\ldots$ & $\ldots$ & 11.0 & -3.77 \\
\hline & 5 & 4368.27 & -1.70 & WF & & $\ldots$ & 38.4 & -3.39 \\
\hline & 9 & 6453.60 & -1.29 & WF & $\ldots$ & & 16.1 & -3.25 \\
\hline & & 6454.44 & -1.07 & WF & & & 23.8 & -3.26 \\
\hline & 10 & 6155.98 & -0.66 & WF & 46.5 & -3.16 & 39.7 & -3.40 \\
\hline & & 6156.77 & -0.44 & WF & 69.3 & -3.11 & 54.7 & -3.39 \\
\hline & & 6158.19 & -0.30 & $\mathrm{WF}$ & 87.7 & -3.06 & 67.0 & -3.36 \\
\hline & 13 & 5018.78 & -2.09 & WF & $\ldots$ & $\ldots$ & 7.0 & -3.03 \\
\hline & & 5019.29 & -1.87 & WF & $\ldots$ & & 10.8 & -3.04 \\
\hline & & 5020.13 & -1.73 & WF & & $\ldots$ & 13.1 & -3.09 \\
\hline & 15 & 4803.00 & -1.98 & $\mathrm{WF}$ & $\ldots$ & $\ldots$ & 9.4 & -3.03 \\
\hline & 16 & 4772.45 & -1.92 & WF & $\ldots$ & $\ldots$ & 2.1 & -3.77 \\
\hline & & 4773.75 & -1.55 & $\mathrm{WF}$ & & $\ldots$ & 8.4 & -3.51 \\
\hline $\mathrm{NaI}$ & & & & & $\mathrm{Na} / \mathrm{N}_{T}=-4.5$ & 0.02 & & $-5.26 \pm 0.02$ \\
\hline & 6 & 5682.63 & -0.70 & WF & & $\ldots$ & 46.9 & -5.29 \\
\hline & & 5688.20 & -0.45 & $\mathrm{WF}$ & $\ldots$ & & 69.7 & -5.24 \\
\hline & 11 & 4751.82 & -2.09 & WF & & & 2.9 & -5.26 \\
\hline Mg I & & & & & $\mathrm{Mg} / \mathrm{N}_{T}=-4.6$ & 0.16 & & -4.67 \\
\hline & 11 & 4702.99 & -0.38 & WS & 6.9 & -4.84 & 111.3 & -4.67 \\
\hline & 15 & 4167.27 & -0.79 & JK & 5.2 & -4.53 & $\ldots$ & $\ldots$ \\
\hline Mg II & & & & & $\mathrm{Mg} / \mathrm{N}_{T}=-4.7$ & 0.08 & & $-4.72 \pm 0.05$ \\
\hline & 5 & 3848.24 & -1.57 & $\mathrm{FW}$ & 40.0 & -4.67 & 42.2 & -4.69 \\
\hline & & 3850.39 & -1.88 & $\mathrm{FW}$ & 26.1 & -4.60 & 30.6 & -4.59 \\
\hline & 9 & 4428.00 & -1.21 & WS & 17.9 & -4.83 & 21.9 & -4.69 \\
\hline & & 4433.99 & -0.91 & $\mathrm{FW}$ & 36.7 & -4.74 & 36.7 & -4.66 \\
\hline & 18 & 4739.59 & -0.64 & $\mathrm{KX}$ & & & 6.7 & -4.89 \\
\hline & 20 & 5401.52 & -0.08 & $\mathrm{KX}$ & 30.1 & -4.71 & $\ldots$ & \\
\hline Al I & & & & & $\log \mathrm{Al} / \mathrm{N}_{T}$ & $-6.08 \pm 0.05$ & & $-5.56 \pm 0.22$ \\
\hline & 1 & 3944.01 & -0.64 & Ws & 27.2 & -6.03 & 175.2 & -5.64 \\
\hline & 2 & 3961.52 & -0.34 & WS & 39.2 & -6.13 & 166.8 & -6.08 \\
\hline Al II & & & & & $\operatorname{g~Al} / \mathrm{N}_{T}=-$ & & & -5.81 \\
\hline Si I & 2 & 4663.10 & -0.28 & FW & $\begin{array}{r}31.0 \\
\log \mathrm{Si} / \mathrm{N}_{T}=\end{array}$ & -6.03 & 16.0 & $\begin{array}{c}-5.81 \\
-4.21 \pm 0.07\end{array}$ \\
\hline & 1 & 5645.67 & -2.14 & VALD2 & & $\ldots$ & 7.5 & -4.17 \\
\hline & & 5665.55 & -2.04 & VALD2 & $\ldots$ & & 9.4 & -4.17 \\
\hline & & 5690.47 & -1.87 & VALD2 & & $\ldots$ & 12.5 & -4.20 \\
\hline & 2 & 5701.10 & -2.05 & VALD2 & $\ldots$ & $\ldots$ & 9.4 & -4.16 \\
\hline & & 5708.43 & -1.47 & VALD2 & $\ldots$ & & 28.9 & -4.16 \\
\hline & 5 & 6145.01 & -1.31 & VALD2 & $\ldots$ & $\ldots$ & 13.4 & -4.22 \\
\hline & & 6155.13 & -0.75 & VALD2 & $\ldots$ & $\ldots$ & 27.8 & -4.41 \\
\hline Si II & & & & & $\mathrm{Si} / \mathrm{N}_{T}=-4.5$ & 0.20 & & $-4.80 \pm 0.23$ \\
\hline & 1 & 3853.66 & -1.44 & LA & 160.0 & -4.58 & 92.0 & -4.78 \\
\hline & & 3856.02 & -0.49 & LA & 290.9 & -4.06 & 143.2 & $\ldots$ \\
\hline & & 3862.59 & -0.74 & LA & 288.5 & -3.83 & 131.8 & $\ldots$ \\
\hline & 2 & 6347.09 & 0.23 & $\mathrm{CR}$ & $\ldots$ & 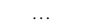 & 204.8 & -4.32 \\
\hline & & 6371.36 & -0.08 & $\mathrm{CR}$ & & & 159.1 & -4.43 \\
\hline & 3 & 4128.02 & -1.40 & CR & 203.0 & -4.08 & 102.4 & -4.69 \\
\hline & & 4130.00 & -1.40 & CR & 210.7 & -4.17 & 95.0 & -4.94 \\
\hline & 5 & 5041.02 & 0.17 & CR & 148.9 & -4.04 & 48.9 & -4.96 \\
\hline & & 5056.00 & 0.44 & CR & & & 79.6 & -4.75 \\
\hline
\end{tabular}

A29 
Table A1: - continued

\begin{tabular}{|c|c|c|c|c|c|c|c|c|}
\hline \multirow[b]{2}{*}{ Species } & \multirow[b]{2}{*}{ Multiplet } & \multirow[b]{2}{*}{$\lambda(\AA)$} & \multirow[b]{2}{*}{$\log g f$} & \multirow[b]{2}{*}{ Ref. } & & & & 0404 \\
\hline & & & & & $\mathrm{W}_{\lambda}(\mathrm{m} \AA)$ & $\log \mathrm{N} / \mathrm{N}_{T}$ & $\mathrm{~W}_{\lambda}(\mathrm{m} \AA)$ & $\log \mathrm{N} / \mathrm{N}_{T}$ \\
\hline Si II & (continued) & & & & & & & \\
\hline & 5 & 5056.35 & -0.53 & ws & 57.8 & -4.26 & 33.8 & -4.53 \\
\hline & - & 4075.45 & -1.40 & SG & 25.0 & -4.66 & 6.8 & 5.05 \\
\hline & 7.05 & 4621.42 & -0.54 & WS & 13.1 & -4.77 & 2.3 & -4.82 \\
\hline & & 4621.72 & -0.38 & ws & 5.7 & -4.56 & 2.6 & -4.93 \\
\hline & 7.15 & 4673.27 & -0.35 & $\mathrm{KX}$ & 8.0 & -4.56 & & $\ldots$ \\
\hline & 7.26 & 4190.70 & -0.35 & KG & 5.8 & -4.75 & 9.9 & $\ldots$ \\
\hline & 7.26 & 4198.13 & -0.30 & KG & 10.9 & -4.77 & $\ldots$ & $\ldots$ \\
\hline S I & & & & & & $\mathrm{s} / \mathrm{N}_{T}=\ldots$ & & $-4.63 \pm 0.08$ \\
\hline & 2 & 4694.11 & -1.77 & $\mathrm{CR}$ & $\ldots$ & $\ldots$ & 19.1 & -4.58 \\
\hline & & 4695.44 & -1.92 & $\mathrm{CR}$ & & $\ldots$ & 14.5 & -4.57 \\
\hline & & 4696.25 & -2.14 & $\mathrm{CR}$ & $\ldots$ & $\ldots$ & 6.3 & -4.74 \\
\hline S II & & & & & $\mathrm{N}_{T}=-5.0$ & & & $-4.82 \pm 0.10$ \\
\hline & 7 & 5032.45 & 0.28 & ws & & & 2.4 & -4.94 \\
\hline & 44 & 4153.09 & 0.62 & Ws & 10.8 & -5.07 & 2.6 & -4.82 \\
\hline & & 4162.67 & 0.78 & WS & 14.2 & - & 3.8 & -4.71 \\
\hline $\mathrm{CaI}$ & & & & & $\mathrm{Ca} / \mathrm{N}_{T}=$ & & & $-5.64 \pm 0.15$ \\
\hline & 2 & 4226.73 & +0.24 & FW & 9.7 & -5.75 & 172.0 & -5.67 \\
\hline & 4 & 4425.44 & -0.36 & ws & $\ldots$ & & 59.3 & -5.63 \\
\hline & & 4434.96 & -0.01 & WS & $\ldots$ & $\ldots$ & 70.1 & -5.85 \\
\hline & & 4455.89 & -0.53 & ws & & $\ldots$ & 54.4 & -5.54 \\
\hline & 5 & 4283.01 & -0.22 & WS & $\ldots$ & & 63.9 & -5.71 \\
\hline & & 4289.36 & -0.30 & Ws & $\ldots$ & $\ldots$ & 54.6 & -5.76 \\
\hline & & 4298.99 & -0.41 & ws & & $\ldots$ & 61.0 & -5.56 \\
\hline & & 4302.53 & 0.28 & ws & $\ldots$ & $\ldots$ & 100.2 & -5.66 \\
\hline & & 4318.63 & -0.21 & Ws & & $\ldots$ & 59.1 & -5.78 \\
\hline & 23 & 4578.56 & -0.56 & ws & $\ldots$ & $\ldots$ & 16.6 & -5.74 \\
\hline & & 4581.40 & -0.34 & Ws & $\ldots$ & $\ldots$ & 24.5 & -5.76 \\
\hline & & 4585.87 & -0.19 & ws & $\ldots$ & $\ldots$ & 33.2 & -5.74 \\
\hline & & 4585.92 & -1.26 & ws & $\ldots$ & & 5.5 & -5.56 \\
\hline & 25 & 4092.63 & -0.84 & $\mathrm{CR}$ & & & 11.7 & -5.57 \\
\hline & & 4094.93 & -0.69 & $\mathrm{CR}$ & & & 12.2 & -5.69 \\
\hline & & 4098.56 & -0.54 & $\mathrm{CR}$ & & & 17.4 & -5.42 \\
\hline & 26 & 3870.50 & -0.84 & $\mathrm{FW}$ & & & 5.3 & -5.68 \\
\hline & & 3875.80 & $\begin{array}{l}-0.69 \\
-0.69\end{array}$ & $\mathrm{FW}$ & & & 17.5 & -5.42 \\
\hline & 34 & 5041.61 & -0.42 & ws & & & 19.7 & -5.68 \\
\hline & 35 & 4878.12 & -0.33 & WS & & & 24.9 & -5.64 \\
\hline & 36 & 4526.92 & -0.43 & ws & & & 17.3 & -5.72 \\
\hline & 37 & 4355.07 & -0.43 & ws & & & 16.9 & -5.71 \\
\hline & 39 & 4108.52 & -0.69 & VALD & & & 7.0 & -5.79 \\
\hline & 51 & 4685.26 & -0.88 & ws & & & 17.7 & -5.12 \\
\hline Ca II & & & & & $\mathrm{a} / \mathrm{N}_{T}=-5.7$ & & & $-5.75 \pm 0.02$ \\
\hline & 15 & 5001.49 & -0.52 & $\mathrm{KX}$ & 6.3 & -5.95 & 61.8 & -5.78 \\
\hline & & 5019.98 & -0.26 & $\mathrm{KX}$ & 21.6 & -5.63 & 81.6 & -5.75 \\
\hline & & 5021.13 & -1.22 & $\mathrm{KX}$ & & $\ldots$ & 22.2 & -5.73 \\
\hline Sc II & & & & & $\mathrm{c} / \mathrm{N}_{T}=-9.3$ & & & $-9.00 \pm 0.20$ \\
\hline & 4 & 3352.04 & -1.87 & VALD & $\ldots$ & & 23.1 & -9.13 \\
\hline & & 3368.93 & -0.29 & VALD & & & 90.2 & -9.14 \\
\hline & 7 & 4246.82 & +0.24 & LD & 42.6 & -9.69 & & \\
\hline & 14 & 4354.59 & -1.58 & LD & & $\ldots$ & 59.7 & -9.01 \\
\hline & & 4374.42 & -0.42 & VALD2 & 22.2 & -9.18 & & \\
\hline & & 4384.81 & -1.61 & VALD2 & & $\ldots$ & 32.2 & -9.45 \\
\hline & & 4400.36 & -0.54 & VALD2 & 9.3 & -9.48 & 144.1 & -8.69 \\
\hline & & 4415.56 & -0.68 & $\mathrm{LD}$ & & $\ldots$ & 120.9 & -8.99 \\
\hline & & 4420.66 & -2.27 & VALD2 & $\ldots$ & & 22.3 & -9.07 \\
\hline & 14 & 4431.35 & -1.97 & VALD & $\ldots$ & $\ldots$ & 37.6 & -8.93 \\
\hline & 15 & 4279.92 & -1.39 & $\mathrm{LD}$ & $\ldots$ & $\ldots$ & 8.3 & -8.94 \\
\hline & 15 & 4294.77 & -1.39 & $\mathrm{LD}$ & $\ldots$ & $\ldots$ & 72.3 & -9.03 \\
\hline & & 4305.71 & -1.30 & LD & & $\ldots$ & 82.9 & -9.05 \\
\hline & & 4314.08 & -0.09 & LD & $\ldots$ & $\ldots$ & 145.3 & -9.33 \\
\hline & & 4320.70 & -0.26 & LD & 24.5 & -9.30 & 144.5 & -9.19 \\
\hline & & 4324.99 & -0.44 & $\mathrm{MF}$ & 17.4 & -9.29 & 131.2 & -9.24 \\
\hline & 24 & 4670.40 & -0.58 & LD & 8.1 & -9.04 & & \\
\hline & 23 & 5031.02 & -0.40 & LD & $\ldots$ & $\ldots$ & 102.1 & -9.10 \\
\hline & 26 & 5239.81 & -0.77 & LD & & $\ldots$ & 69.1 & -9.16 \\
\hline & 28 & 6245.63 & -1.02 & VALD2 & $\ldots$ & & 52.7 & -9.14 \\
\hline & & 6279.75 & -1.25 & VALD2 & $\ldots$ & $\ldots$ & 34.9 & -9.17 \\
\hline & & 6300.69 & -1.62 & VALD2 & $\ldots$ & $\ldots$ & 10.6 & -9.13 \\
\hline & & 6309.92 & -1.62 & VALD2 & $\ldots$ & $\ldots$ & 9.6 & -9.46 \\
\hline & & 6320.85 & -1.82 & VALD2 & & $\ldots$ & 13.7 & -9.09 \\
\hline & 29 & 5641.00 & -1.13 & VALD & $\ldots$ & $\ldots$ & 56.8 & -9.02 \\
\hline & & 5657.89 & -0.60 & VALD & $\ldots$ & $\ldots$ & 104.8 & -9.00 \\
\hline & & 5658.36 & -1.21 & VALD & $\ldots$ & $\ldots$ & 42.4 & -9.08 \\
\hline & & 5667.14 & -1.31 & VALD & $\ldots$ & & 44.8 & -8.97 \\
\hline & & 5669.04 & -1.20 & VALD & $\ldots$ & $\ldots$ & 77.7 & -8.70 \\
\hline & & 5684.20 & -1.07 & VALD & & $\ldots$ & 57.4 & -9.00 \\
\hline & 31 & 5526.90 & -0.02 & VALD & 8.0 & -9.42 & 124.5 & -9.10 \\
\hline
\end{tabular}

A30 
Table A1: - continued

\begin{tabular}{|c|c|c|c|c|c|c|c|c|}
\hline \multirow[b]{2}{*}{ Species } & & & & & & 057 & & \\
\hline & Multiplet & $\lambda(\AA)$ & $\log g f$ & Ref. & $\mathrm{W}_{\lambda}(\mathrm{m} \AA)$ & $\log \mathrm{N} / \mathrm{N}_{T}$ & $\mathrm{~W}_{\lambda}(\mathrm{m} \AA)$ & $\log \mathrm{N} / \mathrm{N}_{T}$ \\
\hline Ti I & & & & & $\log \mathrm{Ti} / \mathrm{N}_{T}=$ & & & $-7.53 \pm 0.21$ \\
\hline & 6 & 4656.46 & -1.35 & ws & $\ldots$ & & 6.2 & -6.63 \\
\hline & & 4667.58 & -1.19 & ws & $\ldots$ & $\ldots$ & 3.5 & -7.03 \\
\hline & & 4681.90 & -1.07 & ws & $\ldots$ & $\ldots$ & 5.2 & -6.96 \\
\hline & 11 & 3982.48 & -1.27 & ws & $\ldots$ & & 10.7 & -6.40 \\
\hline & 12 & 3981.76 & -0.35 & ws & $\ldots$ & $\ldots$ & 56.4 & -6.37 \\
\hline & & 3998.63 & -0.06 & ws & $\ldots$ & $\ldots$ & 30.0 & -7.04 \\
\hline & & 3989.75 & -0.20 & WS & $\ldots$ & & 50.9 & -6.58 \\
\hline & & 3964.26 & -1.18 & ws & & $\ldots$ & 3.5 & -6.98 \\
\hline & 13 & 3924.52 & -0.94 & ws & $\ldots$ & & 6.7 & -6.92 \\
\hline & & 3929.87 & -1.06 & ws & $\ldots$ & & 4.8 & -6.97 \\
\hline & & 3948.67 & -0.47 & ws & $\ldots$ & & 21.2 & -6.85 \\
\hline & & 3956.33 & -0.45 & ws & $\ldots$ & & 14.6 & -7.05 \\
\hline & 38 & 4981.73 & +0.50 & ws & $\ldots$ & $\ldots$ & 30.6 & -7.07 \\
\hline & & 4991.06 & +0.38 & ws & $\ldots$ & $\ldots$ & 38.4 & -6.53 \\
\hline & & 4999.50 & +0.25 & ws & $\ldots$ & & 25.1 & -6.95 \\
\hline & & 5007.20 & +0.11 & ws & $\ldots$ & & 55.7 & -6.32 \\
\hline & & 5014.27 & +0.11 & ws & $\ldots$ & & 19.5 & -6.95 \\
\hline & & 5016.16 & -0.57 & WS & $\ldots$ & $\ldots$ & 3.0 & -7.12 \\
\hline & & 5022.87 & -0.43 & ws & $\ldots$ & $\ldots$ & 3.9 & -7.16 \\
\hline & & 5024.84 & -0.60 & ws & $\ldots$ & & 6.4 & -7.14 \\
\hline & 42 & 4512.73 & -0.48 & ws & $\ldots$ & $\ldots$ & 10.5 & -6.61 \\
\hline & & 4518.02 & -0.33 & ws & $\ldots$ & $\ldots$ & 14.1 & -6.63 \\
\hline & & 4533.23 & 0.48 & WS & $\ldots$ & & 26.3 & -7.10 \\
\hline & & 4534.77 & 0.28 & ws & & $\ldots$ & 19.2 & -7.08 \\
\hline & & 4535.57 & 0.16 & VALD & $\ldots$ & & 11.7 & -7.21 \\
\hline & & 4535.91 & -0.04 & VALD & $\ldots$ & $\ldots$ & 6.8 & -7.27 \\
\hline & & 4536.04 & -0.13 & VALD & $\ldots$ & $\ldots$ & 7.6 & -7.13 \\
\hline & & 4548.76 & -0.35 & WS & $\ldots$ & & 4.6 & -7.13 \\
\hline & & 4552.45 & -0.34 & ws & & $\ldots$ & 13.5 & -6.64 \\
\hline & & 4555.48 & -0.49 & ws & $\ldots$ & $\ldots$ & 9.1 & -6.67 \\
\hline & 44 & 4287.40 & -0.44 & ws & $\ldots$ & $\ldots$ & 8.5 & -6.75 \\
\hline & & 4289.06 & -0.38 & ws & $\ldots$ & & 8.5 & -6.81 \\
\hline & & 4290.92 & -0.43 & ws & $\ldots$ & & 12.3 & -6.59 \\
\hline & & 4295.75 & -0.45 & ws & $\ldots$ & $\ldots$ & 5.9 & -6.91 \\
\hline & & 4301.08 & 0.26 & ws & $\ldots$ & & 18.7 & -7.05 \\
\hline & & 4305.91 & 0.51 & VALD & $\ldots$ & & 32.3 & -6.84 \\
\hline & 80 & 4078.47 & -0.12 & VALD & $\ldots$ & & 5.8 & -6.86 \\
\hline & 160 & 4449.14 & +0.50 & WS & $\ldots$ & & 5.6 & -7.03 \\
\hline & & 4450.89 & +0.41 & ws & $\ldots$ & $\ldots$ & 5.3 & -7.24 \\
\hline & & 4617.25 & +0.39 & ws & $\ldots$ & $\ldots$ & 5.3 & -6.71 \\
\hline Ti II & & & & & $\mathrm{Ti} / \mathrm{N}_{T}=-7.4$ & 0.18 & & $-7.14 \pm 0.20$ \\
\hline & 11 & 3981.99 & -2.91 & PT & 9.8 & -7.02 & 93.7 & -6.78 \\
\hline & & 3987.60 & -2.93 & PT & 2.0 & -7.68 & 72.6 & -7.07 \\
\hline & & 4012.38 & -1.84 & PT & 62.4 & -7.14 & & \\
\hline & & 4025.13 & -2.14 & PT & 30.5 & -7.22 & 101.6 & -7.39 \\
\hline & 12 & 3813.38 & -1.83 & PT & 23.6 & -7.64 & & $\ldots$ \\
\hline & & 3814.58 & -1.61 & PT & 40.1 & -7.61 & $\ldots$ & $\ldots$ \\
\hline & 18 & 4469.14 & -2.33 & PT & 6.2 & -7.51 & 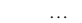 & $\ldots$ \\
\hline & 19 & 4450.49 & -1.52 & PT & $\ldots$ & $\ldots$ & 134.3 & -7.14 \\
\hline & 20 & 4287.89 & -1.79 & PT & & & 108.1 & -7.33 \\
\hline & & 4294.09 & -0.93 & PT & 83.6 & -7.55 & & \\
\hline & & 4344.24 & -1.91 & PT & $\ldots$ & $\ldots$ & 103.4 & -7.30 \\
\hline & 21 & 4161.53 & -2.09 & PT & $\ldots$ & & 86.7 & -7.36 \\
\hline & & 4173.53 & -1.88 & PT & $\ldots$ & & & \\
\hline & & 4184.33 & -2.50 & PT & $\ldots$ & & 92.1 & -6.88 \\
\hline & & 4190.23 & -2.89 & PT & $\ldots$ & & 31.2 & -7.39 \\
\hline & 29 & 4794.84 & -4.19 & VALD2 & & $\ldots$ & 2.5 & -7.31 \\
\hline & & 4849.18 & -3.00 & PT & $\ldots$ & & 47.1 & -7.05 \\
\hline & & 4865.62 & -2.79 & PT & $\ldots$ & $\ldots$ & 50.5 & -7.22 \\
\hline & 30 & 4506.74 & -3.49 & VALD2 & $\ldots$ & $\ldots$ & 16.7 & -7.11 \\
\hline & & 4552.29 & -2.89 & VALD2 & $\ldots$ & $\ldots$ & 50.1 & -7.10 \\
\hline & 39 & 4549.81 & -2.35 & PT & & $\ldots$ & 100.6 & -6.88 \\
\hline & & 4583.41 & -2.92 & PT & $\ldots$ & & 55.8 & -6.96 \\
\hline & & 4609.26 & -3.43 & VALD2 & & & 21.8 & -7.01 \\
\hline & 31 & 4501.27 & -0.77 & PT & 88.0 & -7.67 & $\ldots$ & $\ldots$ \\
\hline & 34 & 3900.56 & -0.20 & PT & 157.5 & -7.47 & $\ldots$ & $\ldots$ \\
\hline & & 3932.01 & -1.64 & PT & 28.6 & -7.41 & & \\
\hline & 40 & 4417.72 & -1.19 & PT & 42.8 & -7.67 & 140.5 & -7.28 \\
\hline & & 4441.73 & -2.33 & PT & 8.4 & -7.31 & 78.1 & -7.22 \\
\hline & & 4464.45 & -1.81 & PT & 16.8 & -7.53 & 118.3 & -7.10 \\
\hline & & 4470.84 & -2.02 & PT & 13.4 & -7.42 & $\ldots$ & $\ldots$ \\
\hline & 41 & 4300.06 & -0.44 & PT & 128.5 & -7.58 & & $\ldots$ \\
\hline & & 4301.93 & -1.15 & PT & 31.8 & -7.86 & 136.9 & -7.37 \\
\hline & & 4307.90 & -1.02 & PT & 74.0 & -7.45 & $\ldots$ & $\ldots$ \\
\hline & & 4312.86 & -1.10 & PT & 58.8 & -7.56 & $\ldots$ & $\ldots$ \\
\hline & & 4314.97 & -1.08 & PT & 62.6 & -7.53 & & \\
\hline & 48 & 4763.88 & -2.36 & PT & & & 75.8 & -7.23 \\
\hline & 50 & 4533.97 & -0.53 & PT & 153.7 & -7.23 & & $\ldots$ \\
\hline & & 4563.76 & -0.69 & PT & 67.7 & -7.87 & $\ldots$ & $\ldots$ \\
\hline & 51 & 4394.06 & -1.78 & PT & 18.2 & -7.48 & $\ldots$ & $\ldots$ \\
\hline & & 4399.77 & -1.19 & PT & 40.6 & -7.65 & & $\cdots$ \\
\hline & & 4407.68 & -2.62 & PT & & & 72.0 & -6.98 \\
\hline & 59 & 4657.21 & -2.24 & PT & 7.1 & -7.45 & & $\ldots$ \\
\hline & 60 & 4544.02 & -2.58 & PT & & & 58.1 & -7.20 \\
\hline
\end{tabular}

A31 
Table A1: - continued

\begin{tabular}{|c|c|c|c|c|c|c|c|c|}
\hline \multirow[b]{2}{*}{ Species } & & & & & HD & & & $\overline{0404}$ \\
\hline & Multiplet & $\lambda(\AA)$ & $\log g f$ & Ref. & $\mathrm{w}_{\lambda}(\mathrm{m} \AA)$ & $\log \mathrm{N} / \mathrm{N}_{T}$ & $\mathrm{~W}_{\lambda}(\mathrm{m} \AA)$ & $\log \mathrm{N} / \mathrm{N}_{T}$ \\
\hline Ti II & (continued) & & & & & & & \\
\hline & 60 & 4568.31 & -2.94 & PT & & & 42.8 & -7.08 \\
\hline & & 4580.45 & -2.94 & PT & 3.2 & -7.11 & 33.9 & -7.21 \\
\hline & & 4600.26 & -3.54 & VALD2 & & & & \\
\hline & 61 & 4391.03 & -2.28 & PT & 5.9 & -7.49 & 100.2 & -6.89 \\
\hline & & 4411.93 & -2.52 & PT & 3.2 & -7.52 & 58.0. & -7.27 \\
\hline & & 4423.24 & -2.67 & $\mathrm{KX}$ & & $\ldots$ & & \\
\hline & 60 & 5154.07 & -1.75 & PT & $\ldots$ & & 97.1 & -7.30 \\
\hline & & 5188.68 & -1.05 & PT & $\ldots$ & $\ldots$ & 130.2 & -7.46 \\
\hline & & 5226.54 & -1.26 & PT & $\cdots$ & $\cdots$ & 163.6 & -6.69 \\
\hline & 72 & 3757.68 & -0.42 & PT & 83.3 & -7.69 & 146.5 & -7.30 \\
\hline & & 3776.05 & -1.25 & PT & 18.8 & -7.71 & & $\ldots$ \\
\hline & 82 & 4529.48 & -1.64 & PT & 19.9 & -7.36 & 106.7 & -7.19 \\
\hline & & 4571.97 & -0.32 & PT & 108.7 & -7.65 & & \\
\hline & 86 & 5129.14 & -1.24 & PT & $\ldots$ & $\ldots$ & 116.1 & -7.26 \\
\hline & & 5131.28 & -3.02 & VALD & $\ldots$ & $\ldots$ & 18.2 & -7.00 \\
\hline & & 5185.90 & -1.49 & PT & & & 104.9 & -7.19 \\
\hline & 87 & 4028.34 & -0.96 & PT & 41.5 & -7.43 & 127.4 & -7.08 \\
\hline & & 4053.83 & -1.13 & PT & & & 140.5 & -6.63 \\
\hline & 92 & 4779.99 & -1.26 & VALD2 & 27.8 & -7.29 & 111.5 & -7.14 \\
\hline & & 4805.09 & -0.96 & VALD2 & 26.3 & -7.61 & 144.8 & -6.84 \\
\hline & 93 & 4374.83 & -1.61 & PT & $\ldots$ & $\ldots$ & 112.3 & -6.70 \\
\hline & & 4421.95 & -1.66 & PT & & $\ldots$ & 78.3 & -7.21 \\
\hline & 94 & 4316.80 & -1.58 & PT & $\ldots$ & $\ldots$ & 78.3 & -7.29 \\
\hline & & 4350.80 & -1.74 & PT & $\ldots$ & $\ldots$ & 63.8 & -7.32 \\
\hline & 101 & 7214.78 & -1.74 & VALD & $\ldots$ & $\ldots$ & 44.7 & -7.26 \\
\hline & & 7355.46 & -1.92 & VALD & $\ldots$ & $\ldots$ & 37.5 & -7.19 \\
\hline & 104 & 4367.66 & -0.86 & PT & $\ldots$ & $\ldots$ & 114.0 & -7.01 \\
\hline & & 4386.84 & -0.96 & PT & & & 92.0 & -7.29 \\
\hline & 105 & 4163.64 & -0.13 & PT & 58.0 & -7.63 & 149.7 & -6.96 \\
\hline & & 4171.90 & -0.29 & PT & 53.2 & -7.52 & 153.2 & -6.72 \\
\hline & & 4174.05 & -1.26 & PT & 13.7 & -7.25 & 67.5 & -7.32 \\
\hline & 106 & 4064.35 & -1.60 & PT & 4.5 & -7.40 & 43.3 & -7.32 \\
\hline & 113 & 5010.21 & -1.29 & PT & $\ldots$ & $\ldots$ & 44.5 & -7.31 \\
\hline & & 5013.68 & -1.91 & PT & $\ldots$ & $\ldots$ & 80.1 & -7.36 \\
\hline & & 5069.09 & -1.82 & PT & $\ldots$ & $\ldots$ & 33.3 & -6.94 \\
\hline & & 5072.28 & -1.06 & PT & & & 69.4 & -7.17 \\
\hline & 114 & 4874.01 & -0.80 & PT & 11.4 & -7.52 & & \\
\hline & & 4911.18 & -0.61 & PT & 18.4 & -7.47 & 90.4 & -7.32 \\
\hline & 115 & 4411.07 & -0.67 & PT & 18.4 & -7.40 & 86.0 & -7.30 \\
\hline & & 4488.34 & -0.51 & PT & 23.5 & -7.43 & 105.9 & -7.12 \\
\hline VI & & & & & $\log \mathrm{V} / \mathrm{N}_{T}=$ & & & $-8.05 \pm 0.11$ \\
\hline & 22 & 4379.24 & 0.58 & MF & $\ldots$ & $\ldots$ & 9.0 & -8.16 \\
\hline & 22 & 4389.97 & 0.20 & $\mathrm{MF}$ & $\ldots$ & $\ldots$ & 7.4 & -7.90 \\
\hline & 29 & 4111.64 & 0.41 & MF & $\ldots$ & $\ldots$ & 6.1 & -8.15 \\
\hline & & 4115.12 & 0.07 & MR & & $\ldots$ & 4.0 & -8.01 \\
\hline V II & & & & & $\mathrm{V} / \mathrm{N}_{T}=-8.38$ & & & $-8.06 \pm 0.18$ \\
\hline & 9 & 3973.64 & -1.07 & BG & $\ldots$ & & 74.1 & -8.35 \\
\hline & & 3997.12 & -1.23 & BG & $\ldots$ & $\ldots$ & 70.6 & -8.21 \\
\hline & & 4002.93 & -1.45 & BG & $\ldots$ &  & 66.5 & -8.09 \\
\hline & & 4036.78 & -1.59 & BG & 5.1 & -8.32 & 53.4 & -8.11 \\
\hline & 10 & 3916.42 & -1.05 & BG & 11.2 & -8.52 & 80.2 & -8.27 \\
\hline & & 3929.73 & -1.59 & BG & $\ldots$ & $\ldots$ & 47.8 & -8.21 \\
\hline & & 3951.96 & -0.74 & BG & $\ldots$ & $\ldots$ & 92.1 & -8.35 \\
\hline & 11 & 3866.74 & -1.55 & BG & & $\ldots$ & 5.44 & -8.14 \\
\hline & & 3903.27 & -0.94 & BG & 14.0 & -8.50 & 99.7 & -8.00 \\
\hline & & 3926.50 & -1.91 & BG & & $\ldots$ & 27.7 & -8.19 \\
\hline & 25 & 4178.39 & -1.57 & $\mathrm{KX}$ & $\ldots$ & $\ldots$ & 36.4 & -8.249 \\
\hline & & 4190.41 & -2.10 & $\mathrm{KX}$ & $\ldots$ & $\ldots$ & 21.6 & -8.02 \\
\hline & & 4209.76 & -1.94 & $\mathrm{KX}$ & & & 36.7 & -7.88 \\
\hline & 32 & 4005.71 & -0.52 & BG & 21.0 & -8.52 & $\ldots$ & $\ldots$ \\
\hline & & 4023.39 & -0.69 & BG & 24.7 & -8.28 & $\ldots$ & $\ldots$ \\
\hline & & 4035.63 & -0.77 & BG & 17.6 & -8.37 & & \\
\hline & 33 & 3878.71 & -0.61 & BG & $\ldots$ & $\ldots$ & 114.3 & -7.76 \\
\hline & & 3884.85 & -1.41 & BG & $\ldots$ & $\ldots$ & 33.0 & -8.34 \\
\hline & & 3914.32 & -0.96 & BG & & $\ldots$ & 89.2 & -7.92 \\
\hline & 37 & 4183.44 & -1.12 & BG & $\ldots$ & $\ldots$ & 75.5 & -7.85 \\
\hline & & 4205.08 & -1.05 & BG & $\ldots$ & $\ldots$ & 68.1 & -8.080 \\
\hline & & 4225.23 & -1.46 & BG & $\ldots$ & $\ldots$ & 55.0 & -8.04 \\
\hline & 56 & 4528.50 & -1.10 & BG & & . & 63.1 & -7.91 \\
\hline & & 4564.59 & -1.39 & BG & 3.9 & -8.18 & 36.9 & -8.01 \\
\hline & & 4600.19 & -1.52 & BG & $\ldots$ & & 44.2 & -7.71 \\
\hline & 199 & 4475.67 & -1.44 & BG & $\ldots$ & $\ldots$ & 4.2 & -7.93 \\
\hline & 255 & 4232.04 & -0.59 & BG & $\ldots$ & $\ldots$ & 17.4 & -7.94 \\
\hline & & 4278.89 & -0.71 & BG & $\ldots$ & & 7.8 & -8.18 \\
\hline $\mathrm{CrI}$ & & & & & $\log \mathrm{Cr} / \mathrm{N}_{T}=$ & & & $-6.40 \pm 0.17$ \\
\hline & 1 & 4254.33 & -0.11 & MF & & $\ldots$ & 111.1 & -6.55 \\
\hline & & 4274.80 & -0.23 & MF & & $\ldots$ & 101.2 & -6.63 \\
\hline & & 4289.72 & -0.36 & MF & $\ldots$ & $\ldots$ & 92.5 & -6.66 \\
\hline & 7 & 5204.50 & -0.21 & MF & $\ldots$ & $\ldots$ & 75.9 & -6.44 \\
\hline & & 5206.02 & +0.02 & MF & $\ldots$ & $\ldots$ & 81.5 & -6.59 \\
\hline & & 5208.42 & +0.16 & MF & $\ldots$ & $\ldots$ & 88.1 & -6.63 \\
\hline & 18 & 5247.56 & -1.64 & MF & & $\ldots$ & 8.3 & -6.32 \\
\hline & & 5264.15 & -1.29 & MF & $\ldots$ & & 8.3 & -6.66 \\
\hline & & 5265.72 & -1.75 & MF & $\ldots$ & $\ldots$ & 8.6 & -6.19 \\
\hline & & 5296.69 & -1.40 & $\mathrm{MF}$ & $\ldots$ & $\ldots$ & 12.5 & -6.35 \\
\hline
\end{tabular}

A32 
Table A1: - continued

\begin{tabular}{|c|c|c|c|c|c|c|c|c|}
\hline \multirow[b]{2}{*}{ Species } & \multirow[b]{2}{*}{ Multiplet } & & & & & & & $\overline{0404}$ \\
\hline & & $\lambda(\AA)$ & $\log g f$ & Ref. & $\mathrm{W}_{\lambda}(\mathrm{m} \AA)$ & $\log \mathrm{N} / \mathrm{N}_{T}$ & $\mathrm{~W}_{\lambda}(\mathrm{m} \AA)$ & $\log \mathrm{N} / \mathrm{N}_{T}$ \\
\hline $\mathrm{CrI}$ & (continued) & & & & $\log \mathrm{Cr} / \mathrm{N}_{T}=$ & & & $-6.40 \pm 0.17$ \\
\hline & 18 & 5298.27 & -1.15 & MF & $\ldots$ & $\ldots$ & 19.7 & -6.38 \\
\hline & & 5300.74 & -2.12 & MF & $\ldots$ & $\ldots$ & 4.9 & -6.07 \\
\hline & & 5345.80 & -0.98 & MF & $\ldots$ & $\ldots$ & 31.1 & -6.29 \\
\hline & & 5348.31 & -1.29 & MF & $\ldots$ & $\ldots$ & 16.8 & -6.30 \\
\hline & & 5409.77 & -0.72 & MF & & & 38.8 & -6.40 \\
\hline & 21 & 4591.39 & -1.74 & MF & $\ldots$ & $\ldots$ & 5.0 & -6.41 \\
\hline & & 4600.75 & -1.26 & MF & $\ldots$ & $\ldots$ & 13.0 & -6.42 \\
\hline & & 4613.37 & -1.68 & MF & $\ldots$ & $\ldots$ & 9.2 & -6.20 \\
\hline & & 4616.12 & -1.19 & MF & $\ldots$ & $\ldots$ & 16.8 & -6.38 \\
\hline & & 4626.18 & -1.32 & MF & & & 13.2 & -6.38 \\
\hline & & 4646.15 & -0.70 & MF & $\ldots$ & $\ldots$ & 32.9 & -6.48 \\
\hline & & 4651.29 & -1.46 & MF & $\ldots$ & $\ldots$ & 9.3 & -6.40 \\
\hline & & 4652.15 & -1.03 & MF & $\ldots$ & $\ldots$ & 22.2 & -6.38 \\
\hline & 22 & 4337.55 & -1.11 & MF & $\ldots$ & $\ldots$ & 14.7 & -6.52 \\
\hline & & 4344.51 & -0.55 & MF & $\ldots$ & & 32.1 & -6.64 \\
\hline & & 4351.05 & -1.45 & MF & & & 17.8 & -6.08 \\
\hline & & 4371.26 & -1.09 & MF & $\ldots$ & $\ldots$ & 18.0 & -6.41 \\
\hline & & 4384.96 & -1.15 & MF & . & $\ldots$ & 16.4 & -6.38 \\
\hline & 23 & 3885.21 & -1.36 & MF & $\ldots$ & $\ldots$ & 9.0 & -6.45 \\
\hline & & 3886.79 & -1.34 & MF & $\ldots$ & $\ldots$ & 3.4 & -6.89 \\
\hline & & 3908.76 & -1.00 & MF & & & 18.7 & -6.43 \\
\hline & & 3919.16 & -0.72 & MF & $\ldots$ & $\ldots$ & 29.7 & -6.44 \\
\hline & & 3941.48 & -1.34 & MF & $\ldots$ & $\ldots$ & 12.5 & -6.28 \\
\hline & 33 & 4526.44 & -0.16 & MF & $\ldots$ & $\ldots$ & 26.2 & -6.00 \\
\hline & & 4530.73 & -0.27 & MF & $\ldots$ & $\ldots$ & 12.8 & -6.26 \\
\hline & & 4535.72 & -0.38 & MF & & & 8.6 & -6.34 \\
\hline & & 4540.48 & -0.49 & MF & $\ldots$ & $\ldots$ & 7.3 & -6.31 \\
\hline & & 4544.60 & -0.58 & MF & & $\ldots$ & 12.9 & -5.95 \\
\hline & 35 & 4126.52 & -0.65 & MF & $\ldots$ & $\ldots$ & 4.6 & -6.33 \\
\hline & 38 & 3963.58 & 0.69 & MF & $\ldots$ & $\ldots$ & 29.0 & -6.73 \\
\hline Cr II & & & & & $\mathrm{Cr} / \mathrm{N}_{T}=-6.4$ & 0.18 & & $-6.56 \pm 0.26$ \\
\hline & 18 & 4112.54 & -3.02 & $\mathrm{KX}$ & & & 18.2 & -6.65 \\
\hline & & 4113.24 & -2.74 & MF & 5.0 & -7.02 & 41.0 & -6.69 \\
\hline & 19 & 4051.97 & -2.19 & MF & 28.2 & -6.76 & 85.2 & -6.57 \\
\hline & & 4087.60 & -3.41 & VALD & & & 20.0 & -6.43 \\
\hline & 20 & 3715.17 & -1.69 & VALD & 137.0 & -6.01 & 124.8 & -6.16 \\
\hline & & 3738.38 & -1.84 & VALD & 59.6 & -6.66 & 109.5 & -6.16 \\
\hline & & 3754.56 & -2.05 & VALD & 39.5 & -6.70 & 30.8 & -6.19 \\
\hline & & 3755.12 & -3.38 & VALD & 6.0 & -6.28 & $\ldots$ & $\ldots$ \\
\hline & & 3765.58 & -2.34 & VALD & 36.0 & -6.46 & $\ldots$ & $\ldots$ \\
\hline & & 3766.63 & -3.20 & VALD & 6.2 & -6.45 & $\ldots$ & $\ldots$ \\
\hline & 21 & 3360.29 & 0.25 & VALD & 150.0 & -6.46 & $\ldots$ & $\ldots$ \\
\hline & & 3361.76 & -1.06 & VALD & 72.1 & -6.39 & $\ldots$ & $\ldots$ \\
\hline & & 3378.33 & -1.05 & VALD & 70.1 & -6.43 & $\ldots$ & $\ldots$ \\
\hline & & 3379.37 & -1.01 & VALD & 75.1 & -6.39 & $\ldots$ & 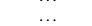 \\
\hline & 23 & 5246.76 & -2.56 & VALD & 8.7 & -6.62 & 49.6 & -6.36 \\
\hline & & 5249.43 & -2.75 & VALD & 10.6 & -6.31 & 42.7 & -6.24 \\
\hline & & 5318.41 & -3.13 & VALD & $\ldots$ & $\ldots$ & 32.0 & -6.07 \\
\hline & & 5346.53 & -3.10 & VALD & & & 28.9 & -6.12 \\
\hline & & 5407.60 & -2.46 & VALD & 10.9 & -6.55 & 49.1 & -6.38 \\
\hline & & 5420.92 & -2.56 & VALD & 10.3 & -6.52 & 41.5 & -6.45 \\
\hline & & 5510.78 & -2.61 & VALD & & $\ldots$ & 45.8 & -6.28 \\
\hline & 24 & 5097.31 & -2.78 & VALD & $\ldots$ & $\ldots$ & 31.8 & -6.42 \\
\hline & & 5116.04 & -3.64 & VALD & & . & 6.7 & -6.33 \\
\hline & & 5153.49 & -2.50 & VALD & 13.0 & -6.47 & 57.9 & -6.26 \\
\hline & & 5210.86 & -2.95 & $\mathrm{KX}$ & 11.0 & -6.10 & 36.8 & -6.13 \\
\hline & & 5305.85 & -2.16 & VALD & 38.8 & -6.22 & & \\
\hline & 26 & 4086.14 & -2.64 & VALD & 9.3 & -6.48 & 34.2 & -6.46 \\
\hline & & 4179.42 & -2.00 & VALD & 46.3 & -6.26 & & \\
\hline & & 4207.36 & -2.73 & VALD & & $\ldots$ & 34.3 & -6.30 \\
\hline & 30 & 4812.34 & -2.13 & MF & 23.7 & -6.48 & 67.5 & -6.41 \\
\hline & & 4824.13 & -1.09 & MF & 132.2 & -6.30 & 120.0 & -6.60 \\
\hline & & 4836.23 & -2.04 & MF & 30.6 & -6.45 & 85.4 & -6.25 \\
\hline & & 4848.24 & -1.05 & $\mathrm{NL}$ & 112.8 & -6.60 & 118.0 & -6.69 \\
\hline & & 4856.19 & -2.17 & VALD & 22.5 & -6.47 & 55.9 & -6.54 \\
\hline & & 4864.32 & -1.47 & VALD & 51.0 & -6.73 & 78.6 & -6.92 \\
\hline & & 4876.41 & -1.38 & VALD & 70.5 & -6.42 & 84.3 & -6.85 \\
\hline & & 4884.61 & -2.23 & VALD & & & 57.0 & -6.46 \\
\hline & 31 & 4242.36 & -1.36 & VALD & 100.7 & -6.31 & 115.3 & -6.32 \\
\hline & & 4252.63 & -2.05 & VALD & 37.2 & -6.31 & 67.0 & -6.46 \\
\hline & & 4269.28 & -2.20 & VALD & 24.1 & -6.39 & 66.0 & -6.33 \\
\hline & & 4275.58 & -1.74 & VALD & 50.9 & -6.45 & 94.5 & -6.34 \\
\hline & & 4284.21 & -1.86 & $\mathrm{KX}$ & & & 79.1 & -6.48 \\
\hline & 39 & 4539.62 & -2.39 & VALD & 11.9 & -6.42 & 41.0 & -6.39 \\
\hline & & 4565.77 & -1.98 & VALD & 20.8 & -6.58 & 72.6 & -6.34 \\
\hline & 43 & 5237.32 & -1.35 & VALD & 90.6 & -6.32 & 114.3 & -6.33 \\
\hline & & 5308.40 & -2.06 & VALD & 19.5 & -6.52 & 63.1 & -6.40 \\
\hline & & 5313.56 & -1.78 & VALD & 52.4 & -6.28 & 74.3 & -6.52 \\
\hline & & 5334.86 & -1.83 & VALD & 36.0 & -6.44 & 94.5 & -6.18 \\
\hline & 44 & 4555.02 & -1.49 & VALD & 67.5 & -6.40 & 107.2 & -6.25 \\
\hline & & 4558.66 & -0.66 & VALD & 177.8 & -6.10 & 123.7 & -6.77 \\
\hline & & 4588.22 & -0.85 & VALD & 116.7 & -6.56 & 134.2 & -6.39 \\
\hline
\end{tabular}

A33 
Table A1: - continued

\begin{tabular}{|c|c|c|c|c|c|c|c|c|}
\hline \multirow[b]{2}{*}{ Species } & \multirow[b]{2}{*}{ Multiplet } & & & & & & & 8404 \\
\hline & & $\lambda(\AA)$ & $\log g f$ & Ref. & $\mathrm{W}_{\lambda}(\mathrm{m} \AA)$ & $\log \mathrm{N} / \mathrm{N}_{T}$ & $\mathrm{~W}_{\lambda}(\mathrm{m} \AA)$ & $\log \mathrm{N} / \mathrm{N}_{T}$ \\
\hline $\mathrm{Cr}$ II & (continued) & & & & $\mathrm{r} / \mathrm{N}_{T}=-6$. & 0.20 & & $-6.56 \pm 0.26$ \\
\hline & 44 & 4592.04 & -1.42 & NL & 51.2 & -6.64 & 97.7 & -6.49 \\
\hline & & 4616.63 & -1.58 & VALD & 45.3 & -6.56 & 91.7 & -6.43 \\
\hline & & 4618.80 & -1.00 & NL & 89.2 & -6.68 & 120.0 & -6.51 \\
\hline & & 4634.07 & -1.24 & MF & 75.5 & -6.57 & 111.0 & -6.44 \\
\hline & 177 & 4697.60 & -1.88 & MF & & & 13.6 & -6.34 \\
\hline & 178 & 4715.12 & -2.31 & MF & & & 6.0 & -6.30 \\
\hline & 179 & 4362.92 & -1.79 & VALD & 4.9 & -6.47 & 13.7 & -6.42 \\
\hline & 180 & 4222.00 & -1.93 & $\mathrm{KX}$ & & & & 0.12 \\
\hline & 183 & 3979.50 & -0.86 & VALD & 32.4 & -6.50 & & \\
\hline & 190 & 4912.46 & -1.26 & VALD & 6.9 & -6.38 & 18.5 & -6.23 \\
\hline & 191 & 4465.73 & -1.28 & VALD & & & 10.8 & -6.58 \\
\hline & 192 & 4256.10 & -1.52 & VALD & 5.7 & -6.20 & 11.6 & -6.21 \\
\hline & 193 & 4049.14 & -1.03 & VALD & 9.7 & -6.45 & 30.9 & -6.18 \\
\hline & & 4070.84 & -0.93 & VALD & 18.3 & -6.25 & 42.4 & -6.07 \\
\hline & 201 & 5076.14 & -1.78 & VALD & & & 2.8 & -6.15 \\
\hline & & 5091.12 & -2.06 & VALD & & & 2.2 & -6.07 \\
\hline & $\mathrm{KX}$ & 4736.99 & -2.78 & VALD & $\ldots$ & $\ldots$ & 17.7 & -6.30 \\
\hline & $\mathrm{KX}$ & 4742.16 & -3.13 & VALD & $\ldots$ & $\ldots$ & 9.9 & -6.20 \\
\hline & $\mathrm{KX}$ & 4779.05 & -1.93 & VALD & $\ldots$ & $\ldots$ & 6.0 & -6.19 \\
\hline Mn I & & & & & $\operatorname{og} \mathrm{Mn} / \mathrm{N}_{T}=$ & & & $-6.56 \pm 0.14$ \\
\hline & 2 & 4030.75 & -0.07 & MF & $\ldots$ & $\ldots$ & 91.7 & -7.02 \\
\hline & & 4033.06 & -0.47 & MF & $\ldots$ & $\ldots$ & 87.8 & -6.94 \\
\hline & & 4034.48 & -0.62 & MF & $\ldots$ & $\ldots$ & 77.4 & -6.64 \\
\hline & 5 & 4018.10 & 0.29 & $\mathrm{BB}$ & $\ldots$ & $\ldots$ & 29.1 & -6.70 \\
\hline & & 4041.55 & 0.29 & MF & & & 91.7 & -7.02 \\
\hline & & 4048.74 & -0.13 & MF & $\ldots$ & $\ldots$ & 64.7 & -6.16 \\
\hline & & 4055.54 & -0.59 & BB & $\ldots$ & $\ldots$ & 87.8 & -6.94 \\
\hline & & 4058.93 & -0.46 & BB & $\ldots$ & $\ldots$ & 18.7 & -6.64 \\
\hline & & 4070.28 & -1.04 & BB & $\ldots$ & $\ldots$ & 10.4 & -6.34 \\
\hline & & 4079.28 & -0.42 & MF & & & 21.7 & -6.62 \\
\hline & & 4079.41 & -0.42 & MF & $\ldots$ & $\ldots$ & 17.0 & -6.71 \\
\hline & & 4082.93 & -0.35 & MF & $\ldots$ & $\ldots$ & 24.4 & -6.60 \\
\hline & & 4083.62 & -0.25 & MF & $\ldots$ & $\ldots$ & 40.4 & -6.41 \\
\hline & 16 & 4754.08 & -0.07 & $\mathrm{BB}$ & $\ldots$ & $\ldots$ & 34.8 & -6.64 \\
\hline & & 4783.42 & 0.06 & BB & $\ldots$ & $\ldots$ & 42.5 & -6.65 \\
\hline & & 4823.52 & 0.15 & BB & & & 48.8 & -6.63 \\
\hline & 17 & 6013.51 & 0.43 & $\mathrm{BB}$ & $\ldots$ & $\ldots$ & 11.2 & -6.33 \\
\hline & & 6016.59 & 0.25 & BB & $\ldots$ & $\ldots$ & 12.9 & -6.44 \\
\hline & & 6021.73 & 0.12 & $\mathrm{BB}$ & $\ldots$ & $\ldots$ & 14.4 & -6.52 \\
\hline & 21 & 4709.71 & -0.49 & BB & $\ldots$ & $\ldots$ & 59.7 & -6.71 \\
\hline & & 4739.08 & -0.60 & BB & $\ldots$ & $\ldots$ & 7.3 & -6.59 \\
\hline & & 4761.51 & -0.14 & $\mathrm{BB}$ & $\ldots$ & $\ldots$ & 20.8 & -6.64 \\
\hline & & 4762.36 & 0.43 & BB & $\ldots$ & $\ldots$ & 49.4 & -6.34 \\
\hline & & 4765.84 & -0.08 & $\mathrm{BB}$ & $\ldots$ & $\ldots$ & 13.5 & -6.66 \\
\hline & & 4766.41 & 0.11 & BB & & & 21.5 & -6.63 \\
\hline Mn II & & & & & $\mathrm{Mn} / \mathrm{N}_{T}=-6$. & 0.14 & & $-6.66 \pm 0.11$ \\
\hline & 2 & 4205.38 & -3.44 & KG & 8.3 & -6.97 & 59.7 & -6.71 \\
\hline & 7 & 4244.25 & -3.44 & KG & & & 7.3 & -6.59 \\
\hline & 5 & 4738.30 & -1.88 & $\mathrm{KX}$ & & & 16.0 & -6.49 \\
\hline & 5 & 4755.73 & -1.24 & $\mathrm{KX}$ & 17.5 & -6.70 & 40.0 & -6.60 \\
\hline & & 4764.73 & -1.35 & $\mathrm{KX}$ & 18.6 & -6.56 & 32.9 & -6.62 \\
\hline & 6 & 4326.64 & -1.36 & KS & 16.3 & -6.62 & 31.0 & -6.64 \\
\hline & & 4343.98 & -1.10 & KS & 14.4 & -6.94 & 34.1 & -6.84 \\
\hline & 7 & 4206.37 & -1.55 & KS & 6.5 & -6.84 & 24.1 & -6.58 \\
\hline & & 4252.96 & -1.14 & $\mathrm{KX}$ & 5.5 & -6.89 & 16.9 & -6.65 \\
\hline & I & 4251.74 & -1.06 & $\mathrm{KX}$ & 9.2 & -6.73 & 18.5 & -6.68 \\
\hline & I & 4791.78 & -1.72 & KX & $\ldots$ & $\ldots$ & 2.9 & -6.89 \\
\hline $\mathrm{Fe} I$ & & & & & $\mathrm{Fe} / \mathrm{N}_{T}=-4.5$ & 0.20 & & $-4.54 \pm 0.17$ \\
\hline & 2 & 4375.93 & -3.03 & $\mathrm{~N} 4$ & $\ldots$ & $\ldots$ & 70.3 & -4.49 \\
\hline & & 4389.25 & -4.58 & N4 & $\ldots$ & $\ldots$ & 4.2 & -4.47 \\
\hline & & 4427.31 & -3.04 & N4 & $\ldots$ & $\ldots$ & 63.6 & -4.54 \\
\hline & & 4461.65 & -3.21 & $\mathrm{~N} 4$ & & & 67.6 & -4.29 \\
\hline & 4 & 3878.57 & -1.38 & N4 & 36.7 & -4.43 & 137.0 & -4.56 \\
\hline & & 3886.28 & -1.08 & N4 & & & 142.7 & -4.73 \\
\hline & & 3899.71 & -1.53 & N4 & 10.0 & -4.93 & 123.7 & -4.486 \\
\hline & & 3920.25 & -1.75 & N4 & 22.3 & -4.31 & 107.3 & -4.91 \\
\hline & & 3922.91 & -1.65 & N4 & 10.6 & -4.81 & 134.3 & -4.42 \\
\hline & & 3927.92 & -1.52 & $\mathrm{~N} 4$ & 12.4 & -4.83 & 119.6 & -4.87 \\
\hline & 15 & 5269.53 & -1.32 & N4 & $\ldots$ & $\ldots$ & 134.8 & -4.38 \\
\hline & & 5328.03 & -1.47 & N4 & $\ldots$ & $\ldots$ & 129.5 & -4.31 \\
\hline & & 5371.48 & -1.65 & N4 & $\ldots$ & $\ldots$ & 108.4 & -4.52 \\
\hline & & 5397.12 & -1.99 & N4 & $\ldots$ & $\ldots$ & 84.5 & -4.64 \\
\hline & & 5405.77 & -1.84 & N4 & $\ldots$ & $\ldots$ & 92.0 & -4.61 \\
\hline & & 5429.69 & -1.88 & N4 & & & 96.4 & -4.52 \\
\hline & & 5434.52 & -2.12 & $\mathrm{~N} 4$ & $\ldots$ & $\ldots$ & 75.5 & -4.58 \\
\hline & & 5446.91 & -1.91 & N4 & $\ldots$ & $\ldots$ & 85.4 & -4.65 \\
\hline & & 5497.51 & -2.85 & N4 & $\ldots$ & $\ldots$ & 36.5 & -4.45 \\
\hline & & 5455.60 & -2.09 & N4 & $\ldots$ & $\ldots$ & 70.7 & -4.69 \\
\hline & & 5501.46 & -3.05 & $\mathrm{~N} 4$ & & & 31.4 & -4.38 \\
\hline & & 5506.77 & -2.80 & $\mathrm{~N} 4$ & $\ldots$ & $\ldots$ & 40.5 & -4.45 \\
\hline & 16 & 4939.68 & -3.34 & VALD & $\ldots$ & $\ldots$ & 19.2 & -4.41 \\
\hline & & 4994.12 & -3.08 & $\mathrm{~N} 4$ & $\ldots$ & $\ldots$ & 26.6 & -4.46 \\
\hline & & 5012.06 & -2.64 & VALD & & & 66.8 & -4.32 \\
\hline
\end{tabular}

A34 
Table A1: - continued

\begin{tabular}{|c|c|c|c|c|c|c|c|c|}
\hline & & & & & & & & 8404 \\
\hline Species & Multiplet & $\lambda(\AA)$ & $\log g f$ & Ref. & $\mathrm{w}_{\lambda}(\mathrm{m} \AA)$ & $\log \mathrm{N} / \mathrm{N}_{T}$ & $\mathrm{~W}_{\lambda}(\mathrm{m} \AA)$ & $\log \mathrm{N} / \mathrm{N}_{T}$ \\
\hline & (continued) & & & & & & & \\
\hline & 16 & 5041.07 & -3.09 & VALD & & & 69.6 & -4.70 \\
\hline & & 5051.63 & -2.80 & $\mathrm{~N} 4$ & $\ldots$ & $\ldots$ & 44.1 & -4.44 \\
\hline & & 5079.73 & -3.22 & $\mathrm{~N} 4$ & & & 24.5 & -4.31 \\
\hline & 20 & 3840.44 & -0.51 & $\mathrm{~N} 4$ & 24.7 & -4.94 & 132.7 & -4.87 \\
\hline & 22 & 3850.82 & -1.73 & $\mathrm{~N} 4$ & 5.4 & -4.44 & 87.2 & -4.65 \\
\hline & 42 & 4147.63 & -2.10 & $\mathrm{~N} 4$ & & & 46.0 & -4.60 \\
\hline & & 4202.02 & -0.71 & $\mathrm{~N} 4$ & 14.3 & -4.74 & & \\
\hline & 42 & 4250.79 & -0.71 & N4 & 14.5 & -4.69 & 113.5 & -4.71 \\
\hline & & 4271.76 & -0.16 & $\mathrm{~N} 4$ & 31.8 & -4.89 & 136.5 & -5.05 \\
\hline & & 4307.90 & -0.07 & $\mathrm{~N} 4$ & & $\ldots$ & 138.8 & -4.93 \\
\hline & & 4325.75 & -0.01 & $\mathrm{~N} 4$ & & & 136.8 & -4.99 \\
\hline & 43 & 4005.25 & -0.61 & $\mathrm{~N} 4$ & 11.4 & -4.88 & 119.5 & -4.555 \\
\hline & & 4063.60 & +0.06 & $\mathrm{~N} 4$ & 40.6 & -4.91 & & \\
\hline & & 4071.74 & -0.02 & $\mathrm{~N} 4$ & 37.1 & -4.85 & 141.3 & -4.78 \\
\hline & & 4132.06 & -0.67 & $\mathrm{~N} 4$ & 18.4 & -4.57 & 106.6 & -4.94 \\
\hline & & 4143.81 & -0.51 & $\mathrm{~N} 4$ & $\ldots$ & $\ldots$ & 122.1 & -4.81 \\
\hline & 68 & 4408.42 & -1.78 & $\mathrm{~N} 4$ & & & 45.8 & -4.42 \\
\hline & & 4430.62 & -1.66 & N4 & $\ldots$ & $\ldots$ & 40.9 & -4.60 \\
\hline & & 4442.34 & -1.26 & $\mathrm{~N} 4$ & $\ldots$ & $\ldots$ & 68.2 & -4.62 \\
\hline & & 4447.72 & -1.34 & $\mathrm{~N} 4$ & $\cdots$ & $\ldots$ & 61.7 & -4.61 \\
\hline & & 4459.12 & -1.28 & $\mathrm{~N} 4$ & $\ldots$ & $\ldots$ & 88.5 & -4.31 \\
\hline & & 4494.57 & -1.14 & $\mathrm{~N} 4$ & $\ldots$ & $\ldots$ & 73.4 & -4.67 \\
\hline & & 4528.61 & -0.82 & $\mathrm{~N} 4$ & & & 90.0 & -4.76 \\
\hline & 71 & 4282.41 & -0.78 & $\mathrm{~N} 4$ & 12.6 & -4.30 & 91.9 & -4.71 \\
\hline & & 4315.08 & -0.97 & $\mathrm{~N} 4$ & t. & $\ldots$ & 88.9 & -4.56 \\
\hline & & 4352.74 & -1.29 & $\mathrm{~N} 4$ & $\ldots$ & $\ldots$ & 55.4 & -4.74 \\
\hline & 72 & 3943.34 & -2.34 & N4 & $\ldots$ & $\ldots$ & 13.3 & -4.50 \\
\hline & & 3949.95 & -1.20 & $\mathrm{~N} 4$ & & & 63.5 & -4.64 \\
\hline & & 3974.76 & -2.61 & N4 & $\ldots$ & $\ldots$ & 9.6 & -4.40 \\
\hline & & 3977.40 & -1.12 & $\mathrm{~N} 4$ & $\ldots$ & $\ldots$ & 78.1 & -4.54 \\
\hline & & 4001.66 & -1.90 & $\mathrm{~N} 4$ & $\ldots$ & $\ldots$ & 34.0 & -4.46 \\
\hline & & 4009.71 & -1.25 & N4 & $\ldots$ & $\ldots$ & 60.7 & -4.66 \\
\hline & & 4030.19 & -2.31 & N4 & & & 18.5 & -4.37 \\
\hline & 73 & 3852.73 & -2.34 & $\mathrm{~N} 4$ & $\ldots$ & $\ldots$ & 56.6 & -4.80 \\
\hline & 115 & 4574.71 & -2.89 & N4 & $\ldots$ & $\ldots$ & 5.7 & -4.34 \\
\hline & & 4630.12 & -2.59 & $\mathrm{~N} 4$ & $\ldots$ & $\ldots$ & 10.8 & -4.34 \\
\hline & 116 & 4439.88 & -3.00 & N4 & $\ldots$ & $\ldots$ & 2.5 & -4.59 \\
\hline & 152 & 4187.04 & -0.55 & N4 & $\ldots$ & & 96.7 & -4.63 \\
\hline & & 4187.80 & -0.55 & N4 & $\ldots$ & $\ldots$ & 115.0 & -4.28 \\
\hline & & 4191.43 & -0.67 & $\mathrm{~N} 4$ & $\ldots$ & $\ldots$ & 101.7 & -4.40 \\
\hline & & 4198.31 & -0.72 & $\mathrm{~N} 4$ & $\ldots$ & $\ldots$ & 112.7 & -4.18 \\
\hline & & 4210.35 & -0.93 & N4 & $\ldots$ & $\ldots$ & 89.9 & -4.36 \\
\hline & & 4222.22 & -0.97 & $\mathrm{~N} 4$ & $\ldots$ & $\ldots$ & 70.8 & -4.65 \\
\hline & & 4233.60 & -0.60 & N4 & & & 97.2 & -4.56 \\
\hline & & 4235.94 & -0.34 & N4 & $\ldots$ & $\ldots$ & 128.3 & -4.21 \\
\hline & & 4250.12 & -0.34 & $\mathrm{~N} 4$ & & $\ldots$ & 102.2 & -4.66 \\
\hline & & 4260.47 & -0.08 & $\mathrm{~N} 4$ & 19.6 & -4.83 & 128.4 & -4.66 \\
\hline & & 4271.15 & -0.35 & $\mathrm{~N} 4$ & 7.6 & -4.83 & 107.8 & -4.63 \\
\hline & & 4299.23 & -0.41 & $\mathrm{~N} 4$ & & & 112.9 & -4.49 \\
\hline & 175 & 3859.21 & -0.75 & $\mathrm{~N} 4$ & 5.2 & -4.57 & & \\
\hline & 278 & 3956.67 & -0.43 & $\mathrm{~N} 4$ & 4.3 & -4.81 & 71.5 & -4.96 \\
\hline & & 3937.33 & -1.46 & N4 & & & 29.6 & -4.59 \\
\hline & & 3997.39 & -0.48 & $\mathrm{~N} 4$ & 8.8 & -4.41 & 103.8 & -4.29 \\
\hline & & 4021.87 & -0.73 & N4 & 6.8 & -4.26 & 61.3 & -4.77 \\
\hline & 280 & 3897.90 & -0.74 & N4 & $\ldots$ & $\ldots$ & 63.7 & -4.76 \\
\hline & & 3907.94 & -1.12 & $\mathrm{~N} 4$ & $\ldots$ & $\ldots$ & 45.0 & -4.61 \\
\hline & 318 & 4871.32 & -0.36 & N4 & $\ldots$ & $\ldots$ & 98.3 & -4.58 \\
\hline & & 4872.14 & -0.57 & N4 & $\ldots$ & $\ldots$ & 79.8 & -4.65 \\
\hline & & 4890.76 & -0.39 & $\mathrm{~N} 4$ & $\ldots$ & $\ldots$ & 94.6 & -4.60 \\
\hline & & 4891.49 & -0.11 & $\mathrm{~N} 4$ & & & 103.6 & -4.74 \\
\hline & & 4918.99 & -0.34 & N4 & $\ldots$ & $\ldots$ & 85.8 & -4.81 \\
\hline & & 4920.50 & +0.07 & N4 & $\ldots$ & $\ldots$ & 117.4 & -4.68 \\
\hline & & 5006.12 & -0.62 & $\mathrm{~N} 4$ & $\ldots$ & $\ldots$ & 74.7 & -4.72 \\
\hline & 350 & 4422.57 & -1.12 & N4 & $\ldots$ & $\ldots$ & 60.5 & -4.38 \\
\hline & & 4443.19 & -1.04 & $\mathrm{~N} 4$ & & & 48.4 & -4.63 \\
\hline & & 4454.38 & -1.04 & $\mathrm{~N} 4$ & $\ldots$ & $\ldots$ & 36.0 & -4.76 \\
\hline & & 4466.55 & -0.60 & $\mathrm{~N} 4$ & $\ldots$ & $\ldots$ & 77.6 & -4.66 \\
\hline & 354 & 4107.49 & -0.88 & N4 & $\ldots$ & $\ldots$ & 47.6 & -4.78 \\
\hline & & 4156.80 & -0.81 & N4 & $\ldots$ & $\ldots$ & 68.8 & -4.55 \\
\hline & & 4175.64 & -0.83 & $\mathrm{~N} 4$ & $\ldots$ & & 59.3 & -4.66 \\
\hline & & 4181.75 & -0.37 & N4 & $\ldots$ & $\ldots$ & 92.6 & -4.60 \\
\hline & 355 & 4154.50 & -0.69 & $\mathrm{~N} 4$ & $\ldots$ & $\ldots$ & 66.9 & -4.69 \\
\hline & & 4184.89 & -0.87 & $\mathrm{~N} 4$ & $\ldots$ & $\ldots$ & 61.9 & -4.59 \\
\hline & & 4191.68 & -1.49 & $\mathrm{~N} 4$ & $\ldots$ & $\ldots$ & 28.0 & -4.50 \\
\hline & & 4203.98 & -1.01 & $\mathrm{~N} 4$ & $\ldots$ & $\ldots$ & 67.1 & -4.37 \\
\hline & & 4213.64 & -1.25 & N4 & $\ldots$ & $\ldots$ & 31.6 & -4.68 \\
\hline & 359 & 4062.44 & -0.86 & N4 & $\ldots$ & $\ldots$ & & $\ldots$ \\
\hline & 383 & 5068.76 & -1.04 & N4 & $\ldots$ & $\ldots$ & 46.1 & -4.62 \\
\hline & & 5139.25 & -0.51 & $\mathrm{~N} 4$ & $\ldots$ & $\ldots$ & 73.6 & -4.68 \\
\hline & & 5191.45 & -0.55 & $\mathrm{~N} 4$ & $\ldots$ & $\ldots$ & 69.6 & -4.68 \\
\hline & & 5192.34 & -0.42 & $\mathrm{~N} 4$ & & & 72.6 & -4.79 \\
\hline & & 5226.86 & -0.56 & $\mathrm{~N} 4$ & $\ldots$ & $\ldots$ & 86.2 & -4.39 \\
\hline & & 5232.93 & -0.06 & $\mathrm{~N} 4$ & $\ldots$ & $\ldots$ & 118.0 & -4.37 \\
\hline & & 5266.55 & -0.39 & N4 & $\ldots$ & $\ldots$ & 74.9 & -4.79 \\
\hline & & 5281.79 & -0.83 & $\mathrm{~N} 4$ & $\ldots$ & $\ldots$ & 55.4 & -4.62 \\
\hline & 430 & 3918.64 & -0.73 & $\mathrm{~N} 4$ & $\ldots$ & $\ldots$ & 55.7 & -4.65 \\
\hline
\end{tabular}


Table A1: - continued

\begin{tabular}{|c|c|c|c|c|c|c|c|c|}
\hline \multirow[b]{2}{*}{ Species } & \multirow[b]{2}{*}{ Multiplet } & & & & & & & \\
\hline & & $\lambda(\AA)$ & $\log g f$ & Ref. & $\mathrm{W}_{\lambda}(\mathrm{m} \AA)$ & $\log \mathrm{N} / \mathrm{N}_{T}$ & $\mathrm{~W}_{\lambda}(\mathrm{m} \AA)$ & $\log \mathrm{N} / \mathrm{N}_{T}$ \\
\hline Fe I & (continued) & & & & & & & \\
\hline & 522 & 4199.10 & +0.16 & $\mathrm{~N} 4$ & 9.6 & -4.84 & 94.5 & -4.83 \\
\hline & 528 & 3843.26 & -0.24 & $\mathrm{~N} 4$ & 4.8 & -4.72 & 71.0 & -4.819 \\
\hline & 664 & 3846.80 & -0.02 & N4 & 4.5 & -4.85 & 71.7 & -4.88 \\
\hline & & 3906.75 & -0.95 & N4 & $\ldots$ & $\ldots$ & 30.5 & -4.62 \\
\hline & 686 & 5615.64 & 0.05 & N4 & $\ldots$ & $\ldots$ & 103.0 & -4.488 \\
\hline & & 5569.61 & -0.49 & $\mathrm{~N} 4$ & $\ldots$ & $\ldots$ & 57.2 & -4.648 \\
\hline & & 5572.84 & -0.28 & $\mathrm{~N} 4$ & $\ldots$ & $\ldots$ & 65.9 & -4.741 \\
\hline & 686 & 5576.09 & -0.94 & N4 & & $\ldots$ & 40.1 & -4.46 \\
\hline & & 5586.75 & -0.14 & $\mathrm{~N} 4$ & $\ldots$ & $\ldots$ & 85.8 & -4.58 \\
\hline & & 5602.94 & -0.85 & $\mathrm{~N} 4$ & $\ldots$ & $\ldots$ & 38.6 & -4.57 \\
\hline & & 5624.54 & -0.76 & $\mathrm{~N} 4$ & $\ldots$ & $\ldots$ & 41.7 & -4.62 \\
\hline & & 5658.53 & -1.86 & $\mathrm{~N} 4$ & $\ldots$ & $\ldots$ & 17.3 & -4.02 \\
\hline & & 5658.81 & -0.84 & $\mathrm{~N} 4$ & $\ldots$ & $\ldots$ & 33.6 & -4.70 \\
\hline & & 5709.37 & -1.03 & $\mathrm{~N} 4$ & $\ldots$ & $\ldots$ & 31.6 & -4.57 \\
\hline & & 5712.13 & -1.99 & $\mathrm{~N} 4$ & $\cdots$ & $\ldots$ & 4.7 & -4.52 \\
\hline & 693 & 4195.22 & -0.49 & VALD & $\ldots$ & $\ldots$ & 60.4 & -4.62 \\
\hline & & 4217.54 & -0.48 & $\mathrm{~N} 4$ & $\ldots$ & $\ldots$ & 53.5 & -4.66 \\
\hline & & 4225.45 & -0.51 & $\mathrm{~N} 4$ & & $\ldots$ & 60.0 & -4.55 \\
\hline & & 4227.43 & 0.27 & $\mathrm{~N} 4$ & $\ldots$ & $\ldots$ & 105.2 & -4.63 \\
\hline & & 4238.82 & -0.23 & N4 & $\ldots$ & $\ldots$ & 83.6 & -4.48 \\
\hline & & 4247.42 & -0.24 & N4 & $\ldots$ & $\ldots$ & 82.8 & -4.51 \\
\hline & 695 & 4114.93 & -1.45 & N4 & $\ldots$ & $\ldots$ & 12.0 & -4.59 \\
\hline & & 4126.18 & -0.92 & $\mathrm{~N} 4$ & & $\ldots$ & 38.3 & -4.52 \\
\hline & & 4150.25 & -0.32 & $\mathrm{~N} 4$ & $\ldots$ & $\ldots$ & 21.0 & -4.53 \\
\hline & & 4153.91 & -0.32 & N4 & $\ldots$ & $\ldots$ & 66.0 & -4.66 \\
\hline & & 4157.78 & -0.40 & N4 & $\ldots$ & $\ldots$ & 55.5 & -4.72 \\
\hline & & 4158.80 & -0.70 & N4 & $\ldots$ & $\ldots$ & 42.0 & -4.61 \\
\hline & & 4176.57 & -0.81 & VALD & & & 54.1 & -4.37 \\
\hline & 801 & 4118.55 & +0.22 & $\mathrm{~N} 4$ & 8.1 & -4.65 & 84.5 & -4.76 \\
\hline & 828 & 4401.29 & -0.89 & N4 & $\ldots$ & $\ldots$ & 32.5 & -4.48 \\
\hline & & 4484.21 & -0.86 & N4 & $\ldots$ & $\ldots$ & 37.1 & -4.43 \\
\hline & 1062 & 5473.90 & -0.79 & N4 & $\ldots$ & $\ldots$ & 21.9 & -4.43 \\
\hline & & 5476.56 & -0.45 & N4 & $\ldots$ & $\ldots$ & 51.9 & -4.28 \\
\hline & & 5563.59 & -0.96 & N4 & $\ldots$ & $\ldots$ & 18.2 & -4.33 \\
\hline & 1065 & 4991.26 & -0.67 & $\mathrm{FW}$ & $\ldots$ & $\ldots$ & 29.1 & -4.36 \\
\hline & 1145 & 5389.47 & -0.41 & VALD & $\ldots$ & $\ldots$ & 28.4 & -4.47 \\
\hline & & 5398.27 & -0.71 & $\mathrm{~N} 4$ & $\ldots$ & $\ldots$ & 19.6 & -4.35 \\
\hline & & 5400.50 & -0.16 & FW & $\ldots$ & $\ldots$ & 46.9 & -4.44 \\
\hline & & 5455.44 & 0.33 & VALD & $\ldots$ & $\ldots$ & 61.5 & -4.76 \\
\hline & & 5461.55 & -1.88 & $\mathrm{~N} 4$ & $\ldots$ & $\ldots$ & 2.8 & -4.09 \\
\hline & & 5546.50 & -1.28 & $\mathrm{~N} 4$ & $\ldots$ & $\ldots$ & 7.7 & -4.29 \\
\hline & 1146 & 5364.85 & 0.23 & N4 & $\ldots$ & $\ldots$ & 55.6 & -4.65 \\
\hline & & 5367.47 & 0.44 & $\mathrm{~N} 4$ & $\ldots$ & $\ldots$ & 64.1 & -4.76 \\
\hline & & 5369.95 & 0.35 & $\mathrm{~N} 4$ & & $\ldots$ & 68.7 & -4.63 \\
\hline & & 5383.36 & 0.65 & N4 & $\ldots$ & $\ldots$ & 82.3 & -4.77 \\
\hline & & 5393.16 & -0.91 & N4 & $\ldots$ & $\ldots$ & 45.8 & -4.55 \\
\hline & & 5424.06 & 0.52 & $\mathrm{~N} 4$ & $\ldots$ & $\ldots$ & 89.3 & -4.53 \\
\hline & 1162 & 5412.80 & -1.72 & FW & $\ldots$ & $\ldots$ & 3.0 & -4.23 \\
\hline & 1163 & 5349.73 & -1.28 & N4 & $\ldots$ & $\ldots$ & 11.6 & -4.09 \\
\hline & & 5445.04 & -0.02 & FW & $\ldots$ & & 44.3 & -4.58 \\
\hline & & 5462.95 & -0.23 & FW & $\ldots$ & $\ldots$ & 41.3 & -4.45 \\
\hline & & 5463.27 & 0.07 & N4 & $\ldots$ & $\ldots$ & 49.1 & -4.59 \\
\hline & & 5562.70 & -0.66 & $\mathrm{FW}$ & $\ldots$ & $\ldots$ & 12.8 & -4.63 \\
\hline Fe II & & & & & $\mathrm{e} / \mathrm{N}_{T}=-4$. & 0.18 & & $-4.62 \pm 0.17$ \\
\hline & 21 & 4177.69 & -3.45 & VALD & & & 108.5 & -4.21 \\
\hline & & 4183.20 & -5.09 & VALD & 4.6 & -4.48 & 16.8 & -4.70 \\
\hline & 22 & 4124.79 & -4.16 & $\mathrm{~N} 4$ & $\ldots$ & $\ldots$ & 55.1 & -4.74 \\
\hline & 25 & 4634.61 & -5.53 & VALD & $\cdots$ & $\ldots$ & 7.6 & -4.67 \\
\hline & & 4648.94 & -4.57 & VALD & 8.1 & -4.79 & 37.9 & -4.79 \\
\hline & & 4670.18 & -4.07 & $\mathrm{~N} 4$ & 20.3 & -4.86 & 68.4 & -4.65 \\
\hline & 26 & 4386.57 & -5.15 & VALD & & & 20.7 & -4.57 \\
\hline & & 4461.43 & -4.37 & VALD & 46.4 & -4.12 & 64.8 & -4.65 \\
\hline & & 4580.06 & -3.73 & VALD & 35.6 & -4.91 & 73.5 & -4.77 \\
\hline & 27 & 4128.74 & -3.58 & $\mathrm{~N} 4$ & 66.8 & -4.66 & $\ldots$ & $\ldots$ \\
\hline & & 4173.46 & -2.16 & N4 & 181.1 & -4.85 & $\ldots$ & $\ldots$ \\
\hline & & 4233.17 & -1.81 & N4 & . & ..278.5 & -4.17 & \\
\hline & & 4416.83 & -2.60 & $\mathrm{~N} 4$ & & & & \\
\hline & & 4665.80 & -5.00 & VALD & 9.2 & -4.23 & 12.6 & -4.77 \\
\hline & 28 & 4087.27 & -4.52 & $\mathrm{~N} 4$ & 9.3 & -4.76 & 32.2 & -4.72 \\
\hline & & 4122.66 & -3.30 & $\mathrm{~N} 4$ & 88.8 & -4.72 & & \\
\hline & & 4258.16 & -3.48 & N4 & 75.2 & -4.61 & 86.4 & -4.81 \\
\hline & & 4369.40 & -3.58 & $\mathrm{~N} 4$ & 48.4 & -4.76 & 82.4 & -4.74 \\
\hline & 29 & 3908.54 & -5.08 & VALD & 6.5 & -4.28 & 11.4 & -4.89 \\
\hline & & 3964.57 & -4.69 & VALD & & & 48.6 & -4.88 \\
\hline & & 3974.16 & -4.05 & $\mathrm{~N} 4$ & 43.7 & -4.38 & 73.5 & -4.42 \\
\hline & 30 & 4833.19 & -4.79 & N4 & 8.0 & -4.49 & 31.6 & -4.42 \\
\hline & & 4839.99 & -4.95 & $\mathrm{~N} 4$ & 3.5 & -4.89 & 23.9 & -4.44 \\
\hline & 32 & 4278.15 & -3.95 & VALD & 40.1 & -4.55 & 93.1 & -4.43 \\
\hline & & 4296.57 & -2.93 & VALD & & & 121.5 & -4.79 \\
\hline & & 4314.31 & -3.60 & VALD & 80.1 & -4.58 & 118.4 & -4.20 \\
\hline & & 4384.33 & -3.68 & $\mathrm{~N} 4$ & & $\ldots$ & 109.0 & -4.23 \\
\hline & & 4413.60 & -4.19 & N4 & & $\ldots$ & 57.4 & -4.60 \\
\hline
\end{tabular}

A36 
Table A1: - continued

\begin{tabular}{|c|c|c|c|c|c|c|c|c|}
\hline & & & & & & 057 & & 404 \\
\hline Species & Multiplet & $\lambda(\AA)$ & $\log g f$ & Ref. & $\mathrm{W}_{\lambda}(\mathrm{m} \AA)$ & $\log \mathrm{N} / \mathrm{N}_{T}$ & $\mathrm{~W}_{\lambda}(\mathrm{m} \AA)$ & $\log \mathrm{N} / \mathrm{N}_{T}$ \\
\hline Fe II & (continued) & & & & & & & \\
\hline & 32 & 4439.13 & -5.50 & VALD & & & 6.2 & -4.50 \\
\hline & 36 & 4993.35 & -3.68 & $\mathrm{~N} 4$ & 38.5 & -4.78 & & \\
\hline & 37 & 4472.92 & -3.53 & $\mathrm{~N} 4$ & 58.3 & -4.66 & 82.1 & -4.75 \\
\hline & & 4489.18 & -2.97 & $\mathrm{~N} 4$ & 122.8 & -4.58 & $\ldots$ & $\ldots$ \\
\hline & & 4491.40 & -2.64 & $\mathrm{~N} 4$ & 152.0 & -4.58 & $\ldots$ & \\
\hline & & 4515.34 & -2.36 & $\mathrm{~N} 4$ & & & 167.8 & -4.21 \\
\hline & & 4520.23 & -2.62 & $\mathrm{~N} 4$ & 109.3 & -5.15 & 148.1 & -4.43 \\
\hline & 37 & 4555.89 & -2.25 & $\mathrm{~N} 4$ & & & 156.3 & -4.65 \\
\hline & & 4582.84 & -3.06 & $\mathrm{~N} 4$ & 98.2 & -4.72 & 107.1 & -4.78 \\
\hline & & 4629.34 & -2.26 & $\mathrm{~N} 4$ & 182.6 & -4.65 & 157.6 & -4.65 \\
\hline & & 4666.76 & -3.33 & N4 & & $\ldots$ & 9.88 & -4.69 \\
\hline & 38 & 4508.28 & -2.35 & $\mathrm{~N} 4$ & & & 173.9 & -4.31 \\
\hline & & 4522.63 & -1.99 & $\mathrm{~N} 4$ & 220.1 & -4.45 & 161.8 & -4.85 \\
\hline & & 4541.52 & -2.97 & $\mathrm{~N} 4$ & 110.5 & -4.69 & 121.9 & -4.58 \\
\hline & & 4576.33 & -2.92 & $\mathrm{~N} 4$ & 110.6 & -4.74 & 118.7 & -4.71 \\
\hline & & 4583.83 & -1.74 & $\mathrm{~N} 4$ & 220.4 & -4.73 & & \\
\hline & & 4595.68 & -4.58 & VALD & 4.8 & -4.85 & 28.6 & -4.89 \\
\hline & & 4620.51 & -3.19 & $\mathrm{~N} 4$ & $\ldots$ & & 105.7 & -4.61 \\
\hline & 39 & 4088.76 & -4.68 & VALD & 4.3 & -4.79 & 20.0 & -4.51 \\
\hline & & 4138.40 & -4.32 & VALD & $\ldots$ & $\ldots$ & 42.3 & -4.42 \\
\hline & 41 & 5256.93 & -4.18 & VALD & $\ldots$ & & 52.3 & -4.65 \\
\hline & & 5284.10 & -3.20 & $\mathrm{~N} 4$ & & & 111.5 & -4.67 \\
\hline & 43 & 4656.97 & -3.57 & $\mathrm{~N} 4$ & 48.4 & -4.71 & 90.7 & -4.56 \\
\hline & & 4731.44 & -3.13 & $\mathrm{~N} 4$ & 76.3 & -4.85 & 112.6 & -4.60 \\
\hline & 44 & 4663.70 & -3.89 & VALD & $\ldots$ & $\ldots$ & 66.9 & -4.65 \\
\hline & 48 & 5264.81 & -3.32 & $\mathrm{~N} 4$ & $\ldots$ & & 97.2 & -4.52 \\
\hline & & 5316.78 & -2.78 & $\mathrm{~N} 4$ & $\ldots$ & $\ldots$ & 148.5 & -4.21 \\
\hline & & 5362.87 & -2.62 & VALD & $\ldots$ & $\ldots$ & 158.3 & -4.30 \\
\hline & & 5414.07 & -3.65 & VALD & & & 62.9 & -4.73 \\
\hline & 49 & 5197.57 & -2.05 & $\mathrm{~N} 4$ & 187.9 & -4.63 & 177.9 & -4.43 \\
\hline & & 5234.62 & -2.21 & $\mathrm{~N} 4$ & $\ldots$ & $\ldots$ & 155.3 & -4.62 \\
\hline & & 5276.00 & -1.90 & $\mathrm{~N} 4$ & $\ldots$ & $\ldots$ & 189.9 & -4.46 \\
\hline & & 5316.61 & -1.85 & $\mathrm{~N} 4$ & $\ldots$ & $\ldots$ & 173.7 & -4.77 \\
\hline & & 5425.25 & -3.39 & $\mathrm{~N} 4$ & . & . & 9.18 & -4.56 \\
\hline & 126 & 4032.94 & -2.57 & VALD & 47.0 & -4.79 & 68.5 & -4.56 \\
\hline & & 4046.81 & -4.37 & VALD & & & 7.4 & -4.53 \\
\hline & 127 & 4024.55 & -2.44 & $\mathrm{~N} 4$ & 90.3 & -4.43 & 76.0 & -4.69 \\
\hline & 141 & 4147.27 & -3.79 & VALD & 5.3 & -4.58 & $\ldots$ & \\
\hline & 150 & 4138.21 & -3.47 & VALD & 15.2 & -4.35 & 21.6 & -4.76 \\
\hline & 151 & 4031.41 & -3.16 & VALD & & & 28.1 & -4.67 \\
\hline & 152 & 3863.38 & -3.51 & VALD & 20.9 & -4.15 & $\ldots$ & $\ldots$ \\
\hline & 153 & 3827.08 & -2.36 & $\mathrm{~N} 4$ & 67.7 & -4.61 & $\ldots$ & 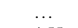 \\
\hline & 167 & 5127.86 & -2.45 & VALD & $\ldots$ & $\ldots$ & 33.6 & -4.55 \\
\hline & & 5160.83 & -2.56 & VALD & $\ldots$ & $\ldots$ & 33.9 & -4.44 \\
\hline & 169 & 4760.15 & -3.64 & VALD & $\ldots$ & & 2.9 & -4.76 \\
\hline & & 4810.74 & -3.29 & VALD & . & . & 8.4 & -4.60 \\
\hline & 171 & 4474.19 & -3.37 & VALD & 7.1 & -4.33 & 9.7 & -4.63 \\
\hline & 172 & 4041.64 & -3.38 & VALD & 5.1 & -4.48 & 16.0 & -4.39 \\
\hline & & 4044.01 & -2.67 & VALD & 21.1 & -4.52 & $\ldots$ & $\ldots$ \\
\hline & & 4048.83 & -2.38 & VALD & 41.5 & -4.45 & $\ldots$ & $\ldots$ \\
\hline & 173 & 3906.04 & -1.70 & $\mathrm{~N} 4$ & 81.9 & -4.64 & $\ldots$ & $\ldots$ \\
\hline & 186 & 4625.91 & -2.55 & VALD & 15.0 & -4.59 & $\ldots$ & \\
\hline & & 4635.33 & -1.58 & $\mathrm{~N} 4$ & 70.3 & -4.66 & $\cdots$ & $\cdots$ \\
\hline & 187 & 4446.25 & -2.78 & VALD & 7.2 & -4.70 & 12.1 & -4.96 \\
\hline & 188 & 4111.90 & -2.67 & VALD & 13.3 & -4.54 & $\ldots$ & $\ldots$ \\
\hline & 190 & 3938.97 & -1.93 & $\mathrm{~N} 4$ & 55.7 & -4.52 & . & . \\
\hline & 205 & 5074.05 & -2.17 & VALD & & & 9.1 & -4.96 \\
\hline & 212 & 3960.90 & -1.56 & VALD & 28.4 & -4.56 & & \\
\hline & 213 & 4354.34 & -1.35 & VALD & 15.8 & -4.85 & 24.2 & -4.51 \\
\hline & & 4507.10 & -1.76 & VALD & 7.9 & -4.63 & $\ldots$ & $\ldots$ \\
\hline & 219 & 4598.53 & -1.54 & VALD & 13.1 & -4.65 & $\ldots$ & $\ldots$ \\
\hline & & 4625.55 & -2.13 & VALD & 3.7 & -4.60 & 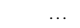 & 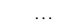 \\
\hline & & 4318.19 & -1.88 & VALD & & & 5.7 & -4.59 \\
\hline & & 4319.68 & -1.64 & VALD & 16.8 & -4.43 & 10.4 & -4.56 \\
\hline & & 4321.31 & -1.74 & VALD & 11.0 & -4.52 & 7.3 & -4.65 \\
\hline & 221 & 5081.90 & -1.06 & VALD & $\ldots$ & $\ldots$ & 2.0 & -4.75 \\
\hline & 222 & 4431.64 & -1.79 & VALD &  & 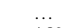 & 8.2 & -4.52 \\
\hline & & 4449.66 & -1.70 & VALD & 6.2 & -4.89 & 10.5 & -4.59 \\
\hline & D & 3844.79 & -1.02 & VALD & 8.3 & -4.77 & & \\
\hline & & 3894.63 & -2.08 & VALD & 4.0 & -4.82 & 13.1 & -4.27 \\
\hline & & 3898.62 & -1.71 & VALD & 12.8 & -4.66 & 13.1 & -4.65 \\
\hline & & 3922.04 & -1.07 & VALD & 5.5 & -4.87 & 5.9 & -4.97 \\
\hline & & 3926.68 & -2.50 & VALD & & & 5.5 & -4.28 \\
\hline & & 4202.52 & -2.36 & VALD & 11.2 & -4.46 & 8.0 & -4.69 \\
\hline & & 4319.42 & -1.99 & VALD & $\ldots$ & $\ldots$ & 6.5 & -4.48 \\
\hline & & 4384.08 & -2.58 & VALD & $\ldots$ & $\ldots$ & 21.6 & -4.34 \\
\hline & & 4418.98 & -1.85 & VALD & $\ldots$ & & 7.1 & -4.51 \\
\hline & & 4467.97 & -2.50 & VALD & $\ldots$ & $\ldots$ & 3.3 & -4.37 \\
\hline & & 4487.50 & -2.14 & VALD & $\ldots$ & $\ldots$ & 6.5 & -4.41 \\
\hline & & 4563.15 & -2.39 & VALD & $\ldots$ & & 2.2 & -4.45 \\
\hline & G & 3924.83 & -1.10 & VALD & $\ldots$ & $\ldots$ & 2.3 & -4.84 \\
\hline & & 4213.52 & -1.84 & VALD & $\ldots$ & $\ldots$ & 8.9 & -4.18 \\
\hline & & 4377.34 & -2.93 & VALD & & $\ldots$ & 3.2 & -4.53 \\
\hline & $\mathrm{J}$ & 4263.87 & -1.69 & VALD & 17.5 & -4.44 & 17.3 & -4.40 \\
\hline & & 4357.58 & -2.01 & VALD & 39.7 & -4.55 & 33.8 & -4.74 \\
\hline & & 4361.25 & -2.26 & VALD & 17.2 & -4.72 & & \\
\hline & & 4402.87 & -2.56 & VALD & & $\ldots$ & 23.2 & -4.38 \\
\hline
\end{tabular}

A37 
Table A1: - continued

\begin{tabular}{|c|c|c|c|c|c|c|c|c|}
\hline \multirow[b]{2}{*}{ Species } & \multirow[b]{2}{*}{ Multiplet } & \multirow[b]{2}{*}{$\lambda(\AA)$} & & & & & & 8404 \\
\hline & & & $\log g f$ & Ref. & $\mathrm{W}_{\lambda}(\mathrm{m} \AA)$ & $\log \mathrm{N} / \mathrm{N}_{T}$ & $\mathrm{~W}_{\lambda}(\mathrm{m} \AA)$ & $\log \mathrm{N} / \mathrm{N}_{T}$ \\
\hline Fe II & (continued) & & & & & & & \\
\hline & $\mathrm{J}$ & 4451.55 & -1.91 & VALD & $\ldots$ & $\ldots$ & 57.1 & -4.42 \\
\hline & & 4455.27 & -2.00 & VALD & $\ldots$ & $\ldots$ & 43.4 & -4.49 \\
\hline & & 4480.68 & -2.56 & VALD & $\cdots$ & & 19.6 & -4.41 \\
\hline & & 4579.53 & -2.34 & VALD & 22.5 & -4.45 & & $\ldots$ \\
\hline & & 4640.84 & -1.74 & VALD & 6.7 & -4.82 & & $\ldots$ \\
\hline & & 4820.85 & -0.72 & VALD & 7.0 & -4.46 & & \\
\hline & & 4824.84 & -2.17 & VALD & 4.8 & -4.57 & 2.2 & -4.55 \\
\hline & & 4836.95 & -2.24 & VALD & & & 4.5 & -4.14 \\
\hline & & 4843.21 & -2.48 & VALD & 6.3 & -4.48 & 6.3 & -4.13 \\
\hline & & 4845.36 & -2.38 & VALD & 3.9 & -4.44 & & 4.15 \\
\hline & & 4883.28 & -0.60 & VALD & 10.7 & -4.36 & 2.9 & -4.68 \\
\hline & & 4893.82 & -4.27 & $\mathrm{~N} 4$ & 9.4 & -4.87 & 43.2 & -4.48 \\
\hline & & 4908.15 & -0.27 & VALD & 9.9 & -4.70 & 5.7 & -4.65 \\
\hline & & 4913.29 & +0.05 & VALD & 20.9 & 4.66 & 8.9 & -4.77 \\
\hline & & 4948.10 & -0.22 & VALD & & $\ldots$ & 4.8 & -4.79 \\
\hline & & 4948.79 & -0.03 & VALD & $\ldots$ & $\ldots$ & 7.4 & -4.74 \\
\hline & & 4951.58 & +0.21 & VALD & $\ldots$ & & 12.0 & -4.53 \\
\hline & & 4984.49 & +0.01 & VALD & 20.4 & -4.60 & 9.2 & -4.67 \\
\hline & & 4990.50 & +0.20 & VALD & & & 9.9 & -4.82 \\
\hline & & 4999.18 & -0.44 & VALD & 7.7 & -4.67 & 5.8 & -4.49 \\
\hline & & 5000.74 & -4.58 & VALD & 7.0 & -4.73 & 33.8 & -4.56 \\
\hline & & 5021.59 & -0.30 & VALD & 20.3 & -4.31 & 11.8 & -4.36 \\
\hline & & 5022.42 & -0.06 & VALD & 9.8 & -4.89 & 7.9 & -4.66 \\
\hline & & 5022.79 & -0.07 & VALD & 28.1 & -4.36 & 9.5 & -4.64 \\
\hline & & 5026.80 & -0.44 & VALD & 14.4 & -4.34 & 5.6 & -4.48 \\
\hline & & 5030.63 & +0.43 & VALD & 39.5 & -4.64 & 19.4 & -4.64 \\
\hline & & 5032.71 & $\begin{array}{l}0.08 \\
+0.08\end{array}$ & VALD & & & 11.1 & $\begin{array}{l}-4.59 \\
-4.59\end{array}$ \\
\hline & & 5035.70 & +0.63 & VALD & 56.4 & -4.56 & 24.4 & -4.74 \\
\hline & & 5070.90 & +0.27 & VALD & & & 10.7 & -4.84 \\
\hline & & 5082.23 & -0.13 & VALD & 13.8 & -4.61 & 5.9 & -4.71 \\
\hline & & 5089.21 & 0.01 & VALD & 11.8 & -4.87 & 6.6 & -4.82 \\
\hline & & 5093.56 & 0.16 & VALD & 33.5 & -4.42 & 9.3 & -4.76 \\
\hline & & 5097.27 & 0.32 & VALD & 36.9 & -4.51 & 13.2 & -4.73 \\
\hline & & 5112.99 & -0.53 & VALD & 4.4 & -4.76 & 1.5 & -4.93 \\
\hline & & 5115.06 & -0.50 & VALD & 3.5 & -4.92 & 3.1 & -4.60 \\
\hline & & 5117.01 & -0.04 & VALD & 9.3 & -4.88 & 5.8 & -4.76 \\
\hline & & 5119.34 & -0.67 & VALD & 2.5 & -4.87 & 2.0 & -4.66 \\
\hline & & 5120.35 & -4.26 & VALD & & & 44.2 & -4.67 \\
\hline & & 5132.66 & -4.09 & $\mathrm{~N} 4$ & 17.0 & -4.79 & 60.8 & -4.62 \\
\hline & & 5143.88 & -0.20 & VALD & 10.6 & -4.65 & 3.7 & -4.80 \\
\hline & & 5144.36 & +0.31 & $\mathrm{~N} 4$ & 25.6 & -4.68 & 11.6 & -4.72 \\
\hline & & 5150.49 & -0.08 & VALD & 12.6 & -4.68 & 4.5 & -4.83 \\
\hline & & 5154.43 & -4.27 & VALD & & & 47.5 & -4.60 \\
\hline & & 5157.28 & -0.17 & VALD & 13.7 & -4.54 & 6.4 & -4.56 \\
\hline & & 5166.56 & -0.05 & VALD & 19.1 & -4.49 & 4.2 & -4.88 \\
\hline & & 5170.78 & -0.33 & VALD & 12.3 & -4.44 & & \\
\hline & & 5180.31 & -0.09 & VALD & 17.5 & -4.53 & 17.5 & -4.13 \\
\hline & & 5199.12 & +0.12 & VALD & 18.1 & -4.72 & 8.1 & -4.77 \\
\hline & & 5203.64 & -0.12 & VALD & & & 7.3 & -4.57 \\
\hline & & 5246.81 & -3.13 & VALD & 72.0 & -4.68 & $\ldots$ & $\ldots$ \\
\hline & & 5337.73 & -3.79 & VALD & 35.0 & -4.47 & & $\ldots$ \\
\hline & & 5362.86 & -2.62 & VALD & 156.8 & -4.36 & $\ldots$ & $\ldots$ \\
\hline Fe III & & & & & $\mathrm{Fe} / \mathrm{N}_{T}=-4.6$ & 0.06 & & $\ldots$ \\
\hline & 4 & 4419.60 & -2.22 & $\mathrm{KX}$ & 11.6 & -4.68 & & $\ldots$ \\
\hline & 5 & 5127.38 & -2.57 & $\mathrm{KX}$ & 5.9 & -4.63 & & $\ldots$ \\
\hline & 9 & 5156.11 & -2.02 & $\mathrm{KX}$ & 6.5 & -4.77 & $\ldots$ & $\cdots$ \\
\hline CoI & & & & & $\ldots$ & & & $-7.17 \pm 0.15$ \\
\hline & 18 & 3873.11 & -0.66 & FW & $\ldots$ & $\ldots$ & 37.6 & -7.04 \\
\hline & & 3873.95 & -0.97 & $\mathrm{FW}$ & $\ldots$ & $\ldots$ & 26.7 & -6.97 \\
\hline & & 3894.07 & 0.10 & $\mathrm{FW}$ & $\ldots$ & $\ldots$ & 59.9 & -6.99 \\
\hline & 29 & 4092.38 & -0.34 & FW & $\ldots$ & $\ldots$ & 6.1 & -7.35 \\
\hline & & 4110.53 & -1.08 & $\mathrm{KX}$ & $\ldots$ & $\ldots$ & 12.5 & -7.19 \\
\hline & 34 & 3845.38 & 0.01 & $\mathrm{FW}$ & $\ldots$ & $\ldots$ & 38.7 & -7.32 \\
\hline & & 3894.97 & -1.40 & $\mathrm{FW}$ & $\cdots$ & $\ldots$ & 3.7 & -7.31 \\
\hline Co II & & & & & $\operatorname{og} \mathrm{Co} / \mathrm{N}_{T}=$ & & & $-6.95 \pm 0.20$ \\
\hline & 1 & 3578.01 & -1.91 & $\mathrm{KX}$ & ... & $\ldots$ & 25.0 & -7.06 \\
\hline & 2 & 3415.77 & -1.74 & $\mathrm{FW}$ & $\ldots$ & $\ldots$ & 45.6 & -6.76 \\
\hline & & 3423.83 & -1.68 & $\mathrm{KX}$ & $\ldots$ & $\ldots$ & 29.6 & -7.16 \\
\hline & & 3446.38 & -1.33 & $\mathrm{KX}$ & & $\ldots$ & 26.4 & -7.11 \\
\hline & & 3501.71 & -1.18 & $\mathrm{KX}$ & 24.9 & -7.16 & 71.1 & -6.68 \\
\hline $\mathrm{Ni} I$ & & & & & $\log \mathrm{Ni} / \mathrm{N}_{T}=$ & & & $-5.73 \pm 0.12$ \\
\hline & 86 & 4401.53 & +0.08 & FW & ... & & 50.1 & -5.84 \\
\hline & & 4462.44 & -0.60 & $\mathrm{FW}$ & $\ldots$ & $\ldots$ & 11.1 & -5.81 \\
\hline & & 4470.47 & -0.40 & $\mathrm{FW}$ & $\ldots$ & $\ldots$ & 18.4 & -5.81 \\
\hline & 98 & 4592.52 & -0.36 & $\mathrm{FW}$ & $\ldots$ & $\ldots$ & 16.9 & $\begin{array}{l}-5.79 \\
-5.79\end{array}$ \\
\hline & & 4600.35 & -0.61 & FW & & $\ldots$ & 15.3 & -5.55 \\
\hline & & 4604.98 & -0.29 & FW & $\ldots$ & & 22.7 & -5.76 \\
\hline & & 4648.64 & -0.16 & FW & $\ldots$ & $\ldots$ & 27.5 & -5.83 \\
\hline & & 4714.40 & 0.23 & $\mathrm{FW}$ & & $\ldots$ & 47.5 & -5.91 \\
\hline & & 4756.51 & -0.34 & FW & $\ldots$ & $\ldots$ & 19.4 & -5.80 \\
\hline & & 4786.53 & -0.17 & $\mathrm{FW}$ & $\ldots$ & $\ldots$ & 44.9 & -5.52 \\
\hline
\end{tabular}

A38 
Table A1: - continued

\begin{tabular}{|c|c|c|c|c|c|c|c|c|}
\hline \multirow[b]{2}{*}{ Species } & \multirow[b]{2}{*}{ Multiplet } & \multirow[b]{2}{*}{$\lambda(\AA)$} & & & $\mathrm{HD}$ & & & $\overline{0404}$ \\
\hline & & & $\log g f$ & Ref. & $\mathrm{w}_{\lambda}(\mathrm{m} \AA)$ & $\log \mathrm{N} / \mathrm{N}_{T}$ & $\mathrm{w}_{\lambda}(\mathrm{m} \AA)$ & $\log \mathrm{N} / \mathrm{N}_{T}$ \\
\hline $\mathrm{Ni} \mathrm{I}$ & (continued) & & & & & & & \\
\hline & 117 & 3908.91 & -0.57 & $\mathrm{KX}$ & $\ldots$ & $\ldots$ & 10.1 & -5.73 \\
\hline & 130 & 4855.40 & 0.00 & $\mathrm{FW}$ & $\ldots$ & $\ldots$ & 25.7 & -5.95 \\
\hline & 131 & 4829.01 & -0.33 & $\mathrm{FW}$ & $\cdots$ & $\ldots$ & 21.6 & -5.71 \\
\hline & 132 & 4752.43 & -0.70 & $\mathrm{FW}$ & $\ldots$ & $\ldots$ & 9.1 & -5.68 \\
\hline & & 4913.96 & -0.63 & $\mathrm{FW}$ & $\ldots$ & $\ldots$ & 9.6 & -5.65 \\
\hline & 163 & 4806.97 & -0.64 & $\mathrm{FW}$ & $\ldots$ & $\ldots$ & 12.9 & -5.57 \\
\hline & 163 & 4546.92 & -0.27 & $\mathrm{KX}$ & $\ldots$ & $\ldots$ & 13.1 & -5.55 \\
\hline $\mathrm{Ni}$ II & & & & & $\mathrm{Ni} / \mathrm{N}_{T}=-5$. & \pm 0.08 & & $-5.92 \pm 0.08$ \\
\hline & 9 & 4244.80 & -3.11 & $\mathrm{KX}$ & 11.9 & -5.90 & 25.2 & -5.82 \\
\hline & & 4362.10 & -2.72 & $\mathrm{KX}$ & 23.9 & -5.94 & 41.6 & -5.92 \\
\hline & 12 & 4015.50 & -2.42 & $\mathrm{KX}$ & 32.7 & -6.07 & 53.0 & -6.02 \\
\hline $\mathrm{ZnI}$ & & & & & $\log \mathrm{Ni} / \mathrm{N}_{T}=$ & & & $-7.61 \pm 0.02$ \\
\hline & 2 & 4680.13 & -0.82 & $\mathrm{KX}$ & $\ldots$ & $\ldots$ & 6.5 & -7.59 \\
\hline & & 4722.15 & -0.34 & $\mathrm{KX}$ & $\ldots$ & $\ldots$ & 16.0 & -7.62 \\
\hline Sr II & & & & & $\mathrm{Sr} / \mathrm{N}_{T}=-9$ & \pm 0.02 & & $-9.20 \pm 0.15$ \\
\hline & 1 & 4077.71 & +0.14 & B & 38.1 & -9.77 & 190.1 & -9.12 \\
\hline & & 4215.52 & -0.18 & B & 22.7 & -9.74 & 159.0 & -9.46 \\
\hline & 3 & 4161.80 & -0.50 & WM & $\ldots$ & $\ldots$ & 37.8 & -9.13 \\
\hline & & 4305.45 & -0.14 & WM & $\ldots$ & & 61.3 & -9.07 \\
\hline Y II & & & & & $\log \mathrm{Y} / \mathrm{N}_{T}=$ & & & $-9.90 \pm 0.14$ \\
\hline & 1 & 4204.69 & -1.76 & $\mathrm{HL}$ & $\ldots$ & $\ldots$ & 25.9 & -9.86 \\
\hline & 5 & 4235.72 & -1.51 & $\mathrm{HL}$ & $\ldots$ & $\ldots$ & 36.8 & -9.81 \\
\hline & & 4309.63 & -0.75 & $\mathrm{HL}$ & $\ldots$ & $\ldots$ & 80.7 & -9.87 \\
\hline & & 4358.72 & -1.32 & $\mathrm{HL}$ & $\ldots$ & $\ldots$ & 41.9 & -9.95 \\
\hline & & 4398.01 & -1.00 & $\mathrm{HL}$ & $\cdots$ & $\ldots$ & 55.5 & -10.05 \\
\hline & 7 & 3788.69 & -0.07 & $\mathrm{HL}$ & $\ldots$ & $\ldots$ & 101.3 & -10.05 \\
\hline & & 3818.34 & -0.98 & $\mathrm{HL}$ & $\ldots$ & $\ldots$ & 54.8 & -9.98 \\
\hline & & 3832.89 & -0.34 & $\mathrm{HL}$ & $\ldots$ & $\ldots$ & 80.6 & -10.17 \\
\hline & 12 & 4682.32 & -1.51 & $\mathrm{HL}$ & $\ldots$ & $\ldots$ & 21.3 & -9.95 \\
\hline & 14 & 4124.91 & -1.50 & $\mathrm{HL}$ & $\ldots$ & $\ldots$ & 32.5 & -9.66 \\
\hline & 16 & 3930.66 & -1.61 & $\mathrm{HL}$ & $\ldots$ & $\ldots$ & 15.9 & -9.91 \\
\hline & 22 & 4854.86 & -0.38 & $\mathrm{HL}$ & $\ldots$ & $\ldots$ & 68.6 & -9.86 \\
\hline & & 4883.68 & +0.07 & $\mathrm{HL}$ & $\ldots$ & $\ldots$ & 87.1 & -9.96 \\
\hline & & 4900.10 & +0.19 & $\mathrm{HL}$ & $\ldots$ & $\ldots$ & 93.3 & -9.74 \\
\hline & 27 & 5497.40 & -0.58 & $\mathrm{HL}$ & $\ldots$ & & 33.1 & -9.64 \\
\hline Zr II & & & & & $\mathrm{g} \mathrm{Zr} / \mathrm{N}_{T}=-9$. & \pm 0.12 & & $-9.42 \pm 0.15$ \\
\hline & 15 & 4096.63 & -1.73 & LN & $\ldots$ & $\ldots$ & 12.5 & -9.43 \\
\hline & & 4211.88 & -0.98 & $\mathrm{LN}$ & $\ldots$ & $\ldots$ & 56.8 & -9.33 \\
\hline & & 4258.05 & -1.20 & LN & & & 52.3 & -9.16 \\
\hline & 16 & 3998.95 & -0.52 & $\mathrm{LN}$ & 3.0 & -9.78 & 78.6 & -9.38 \\
\hline & 29 & 4090.52 & -1.10 & LN & & & 33.7 & -9.38 \\
\hline & 30 & 3991.15 & -0.30 & $\mathrm{BG}$ & 5.6 & -9.59 & 72.6 & -9.55 \\
\hline & & 4161.21 & -0.59 & LN & 4.1 & -9.48 & 62.2 & -9.48 \\
\hline & 40 & 4317.29 & -1.38 & LN & $\ldots$ & & 25.0 & -9.33 \\
\hline & & 4496.98 & -0.89 & LN & $\ldots$ & $\ldots$ & 45.6 & -9.48 \\
\hline & 41 & 4208.97 & -0.51 & LN & & $\ldots$ & 58.3 & -9.63 \\
\hline & 43 & 4050.32 & -1.06 & LN & $\ldots$ & $\ldots$ & 36.0 & -9.41 \\
\hline & 67 & 4461.25 & -1.13 & $\mathrm{LN}$ & $\ldots$ & $\ldots$ & 32.9 & -9.21 \\
\hline & & 4613.94 & -1.54 & LN & $\ldots$ & $\ldots$ & 14.7 & -9.28 \\
\hline & 79 & 4370.96 & -0.77 & LN & $\ldots$ & $\ldots$ & 32.7 & -9.41 \\
\hline & & 4414.54 & -1.08 & LN & $\ldots$ & $\ldots$ & 20.7 & -9.34 \\
\hline Ba II & & & & & $\log \mathrm{Ba} / \mathrm{N}_{T}=$ & & & $-9.54 \pm 0.09$ \\
\hline & 1 & 4554.03 & +0.16 & DS & 7.0 & -10.11 & 143.8 & -9.44 \\
\hline & & 4934.07 & -0.16 & DS & $\ldots$ & $\ldots$ & 137.3 & -9.40 \\
\hline & 2 & 5853.66 & -1.11 & DS & $\ldots$ & & 42.0 & -9.69 \\
\hline & & 6141.71 & -0.23 & DS & $\ldots$ & $\ldots$ & 99.9 & -9.53 \\
\hline & & 6496.89 & -0.47 & DS & & $\ldots$ & 105.6 & -9.49 \\
\hline La II & & & & & $\log \mathrm{La} / \mathrm{N}_{T}=$ & & & $-10.77 \pm 0.08$ \\
\hline & 7 & 4921.77 & -0.45 & LB & $\ldots$ & $\ldots$ & 17.2 & -10.72 \\
\hline & 12 & 3790.82 & +0.03 & LB & $\ldots$ & $\ldots$ & 41.1 & -10.65 \\
\hline & & 3794.77 & +0.21 & LB & $\ldots$ & $\ldots$ & 34.3 & -10.86 \\
\hline & & 3849.00 & -0.45 & LB & & $\ldots$ & 19.9 & -10.72 \\
\hline & 13 & 3871.63 & -0.13 & LB & $\ldots$ & $\ldots$ & 27.6 & -10.76 \\
\hline & 24 & 4333.74 & -0.06 & LB & $\ldots$ & $\ldots$ & 24.2 & -10.93 \\
\hline & 26 & 3995.74 & -0.06 & LB & $\ldots$ & $\ldots$ & 28.9 & -10.79 \\
\hline & 40 & 3988.51 & +0.49 & LB & $\ldots$ & $\ldots$ & 38.5 & -10.70 \\
\hline & 41 & 3949.10 & +0.49 & LB & $\ldots$ & $\ldots$ & 27.6 & -11.00 \\
\hline & & 4077.35 & -0.06 & LB & $\ldots$ & $\ldots$ & 25.2 & -10.83 \\
\hline & & 4123.23 & +0.13 & LB & $\ldots$ & $\ldots$ & 30.7 & -10.84 \\
\hline & & 4196.55 & -0.30 & LB & $\ldots$ & $\ldots$ & 20.2 & -10.65 \\
\hline & 66 & 4042.90 & 0.27 & $\mathrm{CB}$ & $\ldots$ & & 19.8 & -10.74 \\
\hline $\mathrm{Ce}$ II & & & & & $\log \mathrm{Ce} / \mathrm{N}_{T}$ & & & $-10.32 \pm 0.21$ \\
\hline & 1 & 4306.72 & -0.23 & MC & $\ldots$ & $\ldots$ & 9.1 & -10.22 \\
\hline & & 4562.36 & 0.19 & PQ & $\ldots$ & $\ldots$ & 12.3 & -10.56 \\
\hline & & 4572.27 & 0.25 & PQ & $\ldots$ & $\ldots$ & 26.4 & -10.06 \\
\hline & & 4628.16 & 0.15 & $\mathrm{PQ}$ & $\ldots$ & $\ldots$ & 10.8 & -10.56 \\
\hline & 2 & 4137.65 & 0.37 & PQ & $\ldots$ & $\ldots$ & 18.1 & -10.49 \\
\hline & & 4142.39 & 0.26 & PQ & $\ldots$ & $\ldots$ & 22.5 & -10.10 \\
\hline & & 4151.97 & 0.25 & $\mathrm{MC}$ & $\ldots$ & $\ldots$ & 24.6 & -10.05 \\
\hline & & 4165.60 & 0.48 & $\mathrm{PQ}$ & & $\ldots$ & 17.4 & -10.29 \\
\hline & & 4460.21 & 0.27 & $\begin{array}{l}\text { PQ } \\
P Q\end{array}$ & $\ldots$ & $\cdots$ & 13.9 & -10.56 \\
\hline
\end{tabular}

A39 
Table A1: - continued

\begin{tabular}{|c|c|c|c|c|c|c|c|c|}
\hline \multirow{3}{*}{$\frac{\text { Species }}{\text { Eu II }}$} & \multirow[b]{2}{*}{ Multiplet } & \multirow[b]{2}{*}{$\lambda(\AA)$} & \multirow[b]{2}{*}{$\log g f$} & \multirow[b]{2}{*}{ Ref. } & \multicolumn{2}{|c|}{ HD 80057} & \multicolumn{2}{|c|}{ HD 80404 } \\
\hline & & & & & $\mathrm{w}_{\lambda}(\mathrm{m} \AA)$ & $\log \mathrm{N} / \mathrm{N}_{T}$ & $\mathrm{w}_{\lambda}(\mathrm{m} \AA \AA)$ & $\log \mathrm{N} / \mathrm{N}_{T}$ \\
\hline & & & & & $\log \mathrm{Eu} / \mathrm{N}_{T}=$ & & & $-11.74 \pm 0.13$ \\
\hline & 1 & 3819.67 & +0.51 & LW & $\ldots$ & $\ldots$ & 24.7 & -11.82 \\
\hline & & & +0.2 & LW & & & & -11.72 \\
\hline & & & +0.21 & LW & 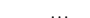 & $\ldots$ & 263 & -1154 \\
\hline & 5 & 3907.10 & +0.17 & LW & & & 8.2 & -11.88 \\
\hline
\end{tabular}

Note: gf value references follow:

$\mathrm{AT}=$ Aldenius et al, 2007); $\mathrm{B}=$ Brage et al, $[1998) \mathrm{BB}=$ Blackwell-Whitehead \& Bergemann 2007):

$\mathrm{BG}=$ Biemont et al. 1 1981, Biemont et al, $\mid$ 1989); $\mathrm{CB}=$ Corliss \& Bozman $\mid$ 1962); $\mathrm{CR}=$ Wiese \& Fuhr [2007)

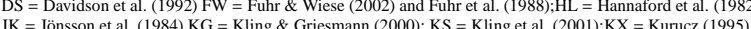

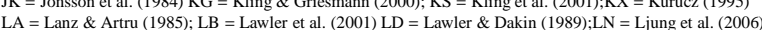

$\mathrm{LW}=$ Lawler et al. $\mid 2001) ; \mathrm{MC}=$ Meggers et al. $\mid 1975) \mathrm{NL}=$ Nilsson et al $[$ [2006



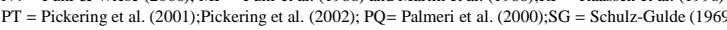

$\mathrm{WF}=$ Wiese et al. [1996); WM = Wiese \& Martin [1980); WS = Wiese et al. 1966

VALD,VALD2 data= Piskunov et al, [1995), Ryabchikova et al. (1997), Kupka et al., 1999

Kupka et al. 2000) 